\title{
Zur Schmetterlingsfauna der lonischen Inseln Griechenlands mit der Beschreibung neuer Psychiden-Taxa sowie ein Beitrag zu ihrer Köcherfliegenfauna (Lepidoptera, Trichoptera)
}

\author{
Mit 16 Figuren, 1 Karte und 2 Tabellen
}

Michael Weidlich ${ }^{1}$

${ }^{1}$ Lindenallee 11, 15898 Neißemünde, OT Ratzdorf, Deutschland. - dr.michael.weidlich@gmail.com Published on 2016-12-20

\section{Zusammenfassung}

Der Autor führte insgesamt 8 Exkursionen zu den Ionischen Inseln in Griechenland durch. Zwei neue Psychidenarten, Bankesia cephalonica spec. nov. und Reisseronia ionica spec. nov. sowie zwei neue Unterarten Reisseronia ionica odysseus subspec. nov. und Reisseronia ionica lefkadensis subspec. nov. werden beschrieben und mit verwandten Arten verglichen. Das erste Taxon wurde im Nationalpark „Enos“ auf Kefalonia auf 1600 m NN entdeckt, $R$. ionica spec. nov. kommt auf Kefalonia, odysseus subspec. nov. auf Ithaka und lefkadensis subspec. nov auf Lefkada vor. Die Taxa von Reisseronia sind auf den Inseln weit verbreitet und stellenweise häufig. Während der Jahre 1993, 2000, 2001, 2002, 2003, 2005, 2012 und 2015 wurde ihre Biologie und Ökologie studiert.

Weiterhin wird der Kenntnisstand zur Lepidopterenfauna der Ionischen Inseln recherchiert und umfassend diskutiert. Im Ergebnis werden 638 Lepidopterenarten bekanntgegeben. Darunter befindet sich eine Art, deren Erstnachweis für Europa mitgeteilt wird und zwei Arten als neu für Griechenland genannt. Für weitere 105 Arten wird deren Erstnachweis für die Ionischen Inseln bekannt gemacht. Ausserdem werden die Beifänge zur Köcherfliegenfauna veröffentlicht.

\section{Key words}

Greece, Ionian Islands, Bankesia cephalonica spec. nov., Reisseronia ionica spec. nov., Reisseronia ionica odysseus subspec. nov., Reisseronia ionica lefkadensis subspec. nov., Psychidae, Lepidoptera, Trichoptera

\section{Summary}

The author have 8 expeditons to the Ionian islands. Two new Psychid species Bankesia cephalonica spec. nov. and Reisseronia ionica spec. nov. and two new subspecies, Reisseronia ionica odysseus subspec. nov. and Reisseronia ionica lefkadensis subspec. nov. are described and compared to related species. The first taxon was found in the Enos National Park on Kefalonia in $1600 \mathrm{~m}$ above sea level and $R$. ionica spec. nov. occur also on Kefalonia, odysseus subspec. nov. on Ithaka and lefkadensis subspec. nov. on Lefkada. The new taxa of Reisseronia occur well distributed and frequently on their respective island. During the years 1993, 2000, 2001, 2002, 2003, 2005, 2012 and 2015 their bionomics have been studied in the habitats.

Furthermore an overview over the Lepidoptera fauna of the Ionian islands is given. From this resulted 638 species as recorded from the Ionian islands, among them is a species recorded for the first time for Europe, two species new for Greece and nevertheless 105 species mentioned for the first time from the Ionian islands. Furthermore additional data of the population of caddisflies (Trichoptera) are published. 


\section{Allgemeiner Teil}

Die Ionischen Inseln sind dem griechischen Festland in der Adria, im sogenannten Ionischen Meer, vorgelagert. Das Ionische Meer bildet das tiefste Becken des Mittelmeeres (4404 m unter NN). Die Inselgruppe besteht aus 7 Hauptinseln Korfu, Paxos, Lefkada, Ithaka, Kefalonia, Zakynthos sowie Kythira. Weiterhin gehören dazu die diapontischen Inseln im NW von Korfu mit den drei bewohnten Inseln, Othoni, Errikousa, Mathraki, dann Pondikonisi im Osten von Korfu, ausserdem Vido und Lazareto und südlich davon liegt Paxos mit der kleineren Insel Antipaxos. Zwischen Lefkada und Arkanien befindet sich die Inselgruppe Tilevoides, worunter Kalamos, Meganisi und Kastos die drei großen, erwähnenswert sind. Zu Ithaka zählen die nordöstlich vorgelagertern Inseln Arkoudi und Atokos. Bei Zakynthos liegen die Strofaden mit Stamfani und Arpyia. Auch die der Peloponnes im Westen vorgelagerte Insel Proti und die sich im Südwesten befindlichen kleineren Inseln, die Echinaden mit Oxia, die Inousses mit Sfaktiria, Sapientza, Schiza und Venetiko sowie im Südosten Elafinissos, Kythira und Antikythira zählen zu den Ionischen Inseln. Der Vollständigkeit halber sei erwähnt, dass es noch etliche kleinere, teilweise unbenannte Ionische Inseln gibt.

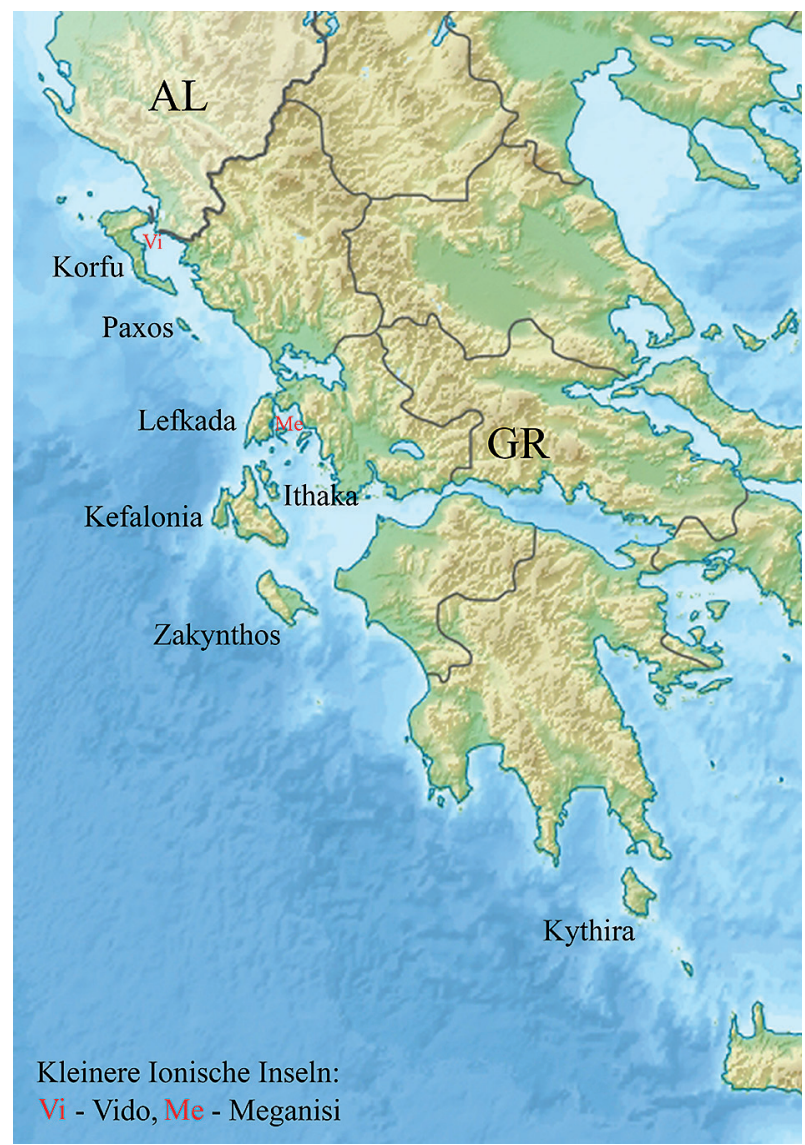

Karte 1: Die Ionischen Inseln Griechenlands im östlichen Mittelmeer, von denen Lepidopterennachweise bekannt sind (verändert nach https://de.wikipedia.org/wiki/Griechenland\#/ media/file:Greece_relief_location_map.jpg).
Geologisch bestehen sie aus Sedimenten (Kalkstein, Dolomit), die hauptsächlich aus dem Mesozoikum stammen. Sie befinden sich an einer bedeutenden tektonischen Linie, die sich durch aktive unterseeische Beben auszeichnet. Dieser tektonische Riß, auch als Hellenischer- oder Kefalonia-Graben bezeichnet, verläuft von Nordwest nach Südost zwischen den Inseln Kefalonia und Zakynthos. Er trennt die eurasische Platte im Nordosten von der afrikanischen Platte in Südwesten. Die Ionischen Inseln liegen auf der eurasischen Platte. Diese Erd- und Seebeben fanden am 12. August 1953 einen traurigen Höhepunkt. So wurde auf Kefalonia fast die Hälfte der Dörfer zerstört. Auch auf Zakynthos gab es große Verwüstungen an baulichen Substanzen. Aktuell sind seit Januar 2014 immer wieder Erdbeben zu verzeichnen, zuletzt am 19.09.2016 mit 4,7 auf der Richterskala (Lokalbeben-Magnitude).

Die Ionischen Inseln sind schon seit dem Altertum besiedelt und die Besiedlung reicht bis in die Bronzezeit zurück. So gilt die Insel Ithaka als die Geburtsinsel des Odysseus. In nachfolgender Zeit zeichnete sich diese Region durch eine oft wechselnde kulturhistorische Besiedlung aus. So gehörten sie dem römischen, später dem oströmischen Reich an und waren im Mittelalter zwischen Neapel und Venedig streitig. Ab 1224 waren die Inseln venezianisch, zwischenzeitlich im 15. und 16. Jahrhundert einige Jahrzehnte türkisch. Ab 1401 bildeten sie die Provinz Levante Veneto und fielen bei deren Zerfall kurzzeitig an Frankreich, Russland, die Türkei und England. 1815 wurden die unabhängigen „Vereinigte Staaten der Ionischen Inseln“ mit einer eigenen Verfassung, die im Jahre 1817 proklamiert wurde, gegründet. Diese standen unter britischem Protektorat. Im Ergebnis des Unabhängigkeitskrieges gegen das osmanische Reich von 1821 bis 1832 beschlossen die europäischen Großmächte die Unabhängigkeit Griechenlands. Großbritannien trat die Inseln dann 1863/1864 an Griechenland ab. Die Ionischen Inseln hatten im 19. Jahrhundert eine wirtschaftliche Spitzenstellung in Griechenland inne, die auch von vielen Völkergruppen aus dem östlichen Mittelmeerraum geprägt wurde.

\section{Ein Überblick zur Erforschung der Schmetterlingsfauna der lonischen Inseln}

Die Erforschung der Insektenfauna des Ionischen Raumes wurde erst relativ spät von Mitteleuropa aus begonnen. STAUdinger (1871) fasst erstmals den wissenschaftlichen Kenntnisstand über die Lepidopterenfauna Griechenlands zusammen. Dieses sind dann auch die ersten zusammenfassenden Angaben über Schmetterlinge von den Ionischen Inseln. Die faunistische Erforschung der einzelnen Inseln ging dann wiederum sehr unterschiedlich vonstatten. So stammen die ersten Angaben von den festlandsnahen Inseln. Korfu wurde seit Mitte des 
19. Jahrhunderts entomologisch besammelt. Überliefert nach Staudinger $(1871: 6,7)$ ist, dass Dr. Theobald KRÜPER (Ückermünde in Pommern/Deutschland) der erste Forscher war, welcher Schmetterlinge auf Korfu in den Jahren 1858 und 1862 beobachtete, sammelte und nach Deutschland mitbrachte. Auch Josef Erber (Wien/ Österreich) sammelte 1866 und 1867 Schmetterlinge auf Korfu (vergl. ERBER, 1866, 1867: 856, STAUdinger, 1871: 10). Ein Jahr später besuchte Erber abermals Korfu wie auch Kythira (= Cerigo), Kefalonia und Zakynthos (Erber, 1867: 856). Dann war es Alfred Hetschko (Komorni Lhotka=Kameral Ellgoth/Tschechien), der eine Ausbeute aus dem Jahre 1881 von der Insel mitbrachte (REBEL, 1924: 39).

Jahrzehnte später wurden verschiedene Funde, hauptsächlich von Tagfaltern auf Korfu, veröffentlicht, so von Norris (1891), MATHEW (1898) (auch von Vido) und De La Garde (1899). Weitere Angaben folgten dann aus den Jahre 1903 von Gustav Paganetti-Hummler (Bad Vöslau bei Wien/Österreich), 1907 Fritz WAGNER (Wien/Österreich) und 1910 Dr. H. Ritter von Woerz (Innsbruck/Österreich) (vergl. ReBEL, 1910: 418). Auch Buresch, I. \& Iltschew besuchten die Insel im Jahr 1909 (Buresch \& IltsCHew, 1915: 46).

Die ersten Aufsammlungen von Kythira, Kefalonia und Zakynthos stammen ebenfalls von Erber, (1867: 856) und werden durch Moritz Peter Joseph Hilf (BosnienHerzegowina) auf Kefalonia 1908 und auf Zakynthos 1909 fortgeführt.

Die Beobachtungen von Lepidopteren auf Lefkada begannen im Jahre 1894 durch Franz Josef Maria Werner (Wien/Österreich).

Rebel (1910) fasst den Kenntnisstand über die lepidopterologische Erforschung der Ionischen Inseln zusammen und kann insgesamt 154 Arten aufzeigen, darunter 75 Macrolepidopterenarten inclusive Psychidae. Weitere faunistische Daten von den Ionischen Inseln werden durch Rebel (1912, 1915, 1924, 1932, 1933, 1937 und 1938) publiziert.

Die aktuelle Literatur über die Inseln wie auch neue Daten zu den Inseln werden von HACKer (1989) und PAMPERIS (2009) (siehe unten) weitgehend dargestellt und deshalb nicht gesondert erwähnt.

Einzelne Schmetterlingsfamilien wurden zwischenzeitlich durchaus umfassend bearbeitet. Monographisch sind die Noctuiden Griechenlands durch Hacker (1989) behandelt worden. Demnach ergibt sich für die Ionischen Inseln folgender Wissenstand: Ionische Inseln Gesamt: 76 Arten

$\begin{array}{lr}\text { Korfu } & 70 \text { Arten } \\ \text { Lefkada } & 5 \text { Arten } \\ \text { Kefalonia } & 7 \text { Arten } \\ \text { Zakynthos } & 3 \text { Arten } \\ \text { Kythira } & 6 \text { Arten }\end{array}$

Von allen anderen Ionischen Inseln sind bis dato keine Eulenarten bekannt geworden.

Für die Insel Korfu gibt es eine provisorische „checklist“ ihrer Tagfalter (BALDOCK \& BRETHERTON, 1981). PAMPERIS (2009) hat monografisch die Tagfalter Griechenlands wie auch die Literatur umfassend bearbeitet und führt 99 Arten für die Ionische Inselgruppe auf. Auch deshalb sollen in vorliegender Arbeit Zitate nur beispielhaft z.B. TOLMANN \& BERNHARD (1994), GASKIN (1996), Papapavlou \& Katsouni (2008) angeführt werden. Ebenfalls werden fragliche Arten aufgenommen, in deren Hinsicht die Quelle unklar oder als nicht gesichert gilt, die aber durchaus nur Beobachtungslücken darstellen und auch auf anderen Ionischen Inseln vorkommen. Lediglich die Arten, die aus zoogeographischer Sicht kaum Faunenelemente der Inselgruppe sein können und auch von PAMPERIs (2009) ausdrücklich als fraglich vermerkt werden, sind weggelassen worden. Für die Ionischen Inseln ergibt sich bis dato für die Tagfalter folgender Kenntnisstand (in Klammern PAMPERIS, 2009: 733).

Ionische Inseln Gesamt: 98 Arten (97)

$\begin{array}{lr}\text { Korfu } & 95 \text { Arten (86) } \\ \text { Lefkada } & 42 \operatorname{Arten}(39) \\ \text { Ithaka } & 3 \text { Arten (3) } \\ \text { Kefalonia } & 59 \text { Arten (59) } \\ \text { Zakynthos } & 39 \text { Arten (35) } \\ \text { Kythira } & 43 \text { Arten (40) } \\ \text { Vido } & 2 \text { Arten (2) } \\ \text { Paxos } & 2 \text { Arten (2) } \\ \text { Meganisi } & 3 \text { Arten (3) } \\ \text { Sapientza } & 1 \text { Art (1) } \\ \text { Elafonissos } & 1 \text { Art (1) } \\ \text { Antikythira } & 4 \text { Arten (4) }\end{array}$

Für alle anderen Schmetterlingsfamilien Griechenlands gibt es bisher keine zusammenfassenden faunistischen Arbeiten, sieht man einmal von der Europaliste von Karsholt \& RAzowski (1996) ab (siehe Tabelle 1).

Diverse Familien der „Microlepidoptera“ wurden im Rahmen der Aufsammlungen von G. Christensen und L. GozmánY in den 1980er Jahren publiziert (vergl. Literaturverzeichnis), jedoch mit nur sehr wenigen Angaben zu den Ionischen Inseln.

In den letzten 25 Jahren sind über die Lepidopterenfauna der Inselgruppe nur wenige Veröffentlichungen (exclusive Tagfalter, siehe PAMPERIS, 2009) erschienen. So stammen Daten von Lefkada, Kefalonia und Kythira von MALICKy (1992), von Zakynthos von EMBACHer (2000), von Korfu, Kefalonia, Ithaka und Lefkada von Hofmann (2003), von Lefkada und Zakynthos von 
De Freina \& Piatkowski (2006) und Piatkowski (2006) und zuletzt PARKer (2010) wiederum über Tagfalter.

Andere, bis dahin nicht publizierte Nachweise konnten aus den Verbreitungskarten der Bestimmungswerke im Rahmen „The Geometrid Moth of Europe " entnommen werden: Archiearinae, Orthostixinae, Desmobathrinae, Alsophilinae, Geometrinae (Hausmann, 2001), Perizomini \& Eupitheciini (Mironov, 2003), Sterrhinae (HAUSMANN, 2004), Larentiinae (HAUsmann \& ViIDALEPP, 2012) und Ennominae (Skou \& SiHVonen, 2015).

Weiterhin konnten etliche Angaben der Internetseite www.lepiforum.de entnommen werden und die Autorenschaft wird mit dem Abrufdatum in Tab. 1 angegeben.

Bis zum heutigen Zeitpunkt gibt es ebenfalls keine ausführliche Arbeit über die Lepidopterenfauna der Ionischen Inseln. Dies bot den Ansatz, alle verfügbaren Daten Ionischer Schmetterlinge zusammenzutragen, möglichst präzise zu beschreiben und zu diskutieren sowie tabellarisch darzustellen.

Das Literaturstudium hat ergeben, dass die Schmetterlingsfauna von Korfu als gut, die von Kefalonia als mittelmäßig, die von Lefkada und Zakynthos als mäßig sowie die von Kythira als mangelhaft angesehen werden kann. Ausserdem bleibt zu bemerken, dass von der grösseren Insel Ithaka bisher lediglich 3 Tagfalterarten (PAMPERIS, 2009: 733) bekannt geworden sind. Von den kleineren Ionischen Inseln liegen fast keine keine Schmetterlingsangaben vor, es sind zumindest keine publiziert worden. Die Ausnahmen bilden lediglich die Inseln Vido mit 2 Tagfalterarten (MATHEW, 1898: 112), Paxos mit einer Sesienart (EMBACHer, 2000: 66) und zwei Tagfalterarten (PAMPERIs, 2009: 766), Meganisi mit drei Pyraliden/Crambiden- und 2 Geometridenarten (Rebel, 1932: 54, 55) sowie 3 Tagfalterarten, Elafonissos mit 1 Tagfalterart und Antikythira mit 4 Tagfalterarten (vergl. PAMPERIS, 2009: 733).

Ausgehend von diesem Kenntnisstand waren die eigenen, interessanten Aufsammlungen Anlass genug, die Fachwelt über diese Ergebnisse zu informieren.

Der Autor besuchte die drei Inseln Ithaka, Kefalonia und Lefkada in den Jahren 1993, 2000, 2001, 2002, 2003, 2005, 2012 und 2015. Diese Expeditionen waren hauptsächlich dem Studium der Psychidenfauna gewidmet. Weitergehend wurden aber auch Macrolepidopteren beobachtet und registriert. Beifänge galten den Microlepidopteren und Trichopteren (hier mit veröffentlicht), Coleopteren und Tipuliden. Die umfangreichsten Ausbeuten stammen von Ithaka.

Zuerst besuchte der Autor Ithaka auf Einladung von Dr. JürGEN RADDE (Vathi, Ithaka) vom 11. bis 16.07.1993, gemeinsam mit Ehefrau Dr. Ilona Weser und Tochter Cornelia Burmeister. Die nächsten Expeditionen wurden jeweils allein durchgeführt, so nach Ithaka und
Lefkada vom 25.04. bis 27.04.2000, Kefalonia und Ithaka vom 30.04. bis 07.05.2001, Lefkada, Ithaka und Kefalonia vom 08.05. bis16.05.2002, Kefalonia, Ithaka und Lefkada vom 30.04. bis 05.05.2003, Kefalonia, Ithaka und Lefkada vom 05.04. bis 09.04.2005, Lefkada vom 21.04. bis 22.04.2012 sowie Lefkada, Kefalonia und Ithaka vom 03.05. bis 07.05.2015.

\section{Bemerkungen zu endemischen Arten bzw. Neubeschreibungen von den Ionischen Inseln}

Rebel (1910: 419) bemerkt, dass die Ionischen Inseln „Ihrem kontinentalen Charakter entsprechend, ...aber keine derselben wirklich endemischen Formen beherbergen... Er führt diese Annahme darauf zurück, dass das naheliegende Festland noch wenig erforscht sein. Gleichwohl beschreibt er zwei für die Wissenschaft neue Microlepidopteren: Ephestia leonhardi, die sich später als Synonym von Euzopherodes lutisignella (MANN, 1869) herausstellte und Argyresthia hilfiella von Kefalonia (Rebel, 1910: 426, 429). Zumindest A. hilfiella scheint endemisch auf Kefalonia vorzukommen, zumindest wird sie ausschließlich aus Griechenland geführt (vergl. Karsholt \& RAzowski, 1996). Später kommt noch eine weitere neue Art hinzu. Die Elachistide Agonopterix leucadensis wird durch ReBel (1932: 55) als eine Depressaria HAwORTH, 1811 nach einem einzelnen $\sigma^{\star}$ aus Nidri (Lefkada) publiziert. Offenbar wurde die Art seitdem nicht mehr gefunden.

Fast 70 Jahre später wird dann eine weitere, für die Wissenschaft neue Art veröffentlicht: Nemapogon scholzi Sutter, 2000, die von Zakynthos nach 5 Ex. und einem weiteren Ex. von Kreta bekannt wurde.

Das der Ansicht von ReBel nur bedingt gefolgt werden kann, zeigen die nachfolgenden Neubeschreibungen von Psychiden. So weisen Lefkada, Ithaka und Kefalonia durchaus endemische Taxa auf und es ist anzunehmen, dass noch weitere Neuentdeckungen folgen.

\section{Der Nationalpark Enos auf Kefalonia}

Kefalonia ist mit 734 Quadratkilometern die größte der Ionischen Inseln und weist mit dem Enos (auch Aenos oder Mega Soros oder Monte Negro) auch den höchsten Berg der Inselgruppe auf. Mit seiner Gipfelhöhe von $1628 \mathrm{~m}$ NN bildet er in einem Umkreis von ca. $75 \mathrm{~km}$ die höchste Erhebung und findet erst auf dem Festland im Akarnanika-Gebirge (Epirus) bzw. $90 \mathrm{~km}$ im Erymanthos-Gebirge auf der Peloponnes vergleichbare Höhen. Geologisch besteht die Insel hauptsächlich aus Kalken der Oberen Kreide sowie im Bereich des Berges Kokini Rachi, nördlich vom Enos gelegen, der Jura und Trias. 
Weitgehend ist die Insel mit Macchienvegetation bedeckt, die sich ausgeprägt als Andrachno-Quercetum und Oleo-Ceratonion-Zone innerhalb der mediterranen immergrünen Hartlaubzone findet, die auch auf den anderen Ionischen Inseln vorherrscht. Einige dieser Gesellschaften ragen auch in den Nationalpark hinein.

Landwirtschaftlich genutzte Flächen sind größtenteils um die Ortslagen herum und in niedrigen Höhen angelegt. Weit verbreitet ist die Beweidung mit Ziegen, die fast auf der gesamten Insel stattfindet, ausgenommen sind verschiedenartig geschützte Plantagen (z.B. Weinanbauflächen) und Flächen im Nationalpark. Der Weinanbau der bekannten Marke Robola findet bis in Höhen von $500 \mathrm{~m}$ NN statt.

Der Nationalpark Enos ist der kleinste griechische Nationalpark und wurde 1962 auf einer Fläche von 2862 ha eingerichtet. Die Gipfelregion ist befahrbar, da hier die Versorgung der Radarstation (ca. 1600 m NN) zu gewährleisten ist. Schutzziel sind die Ökosysteme mit den Vorkommen der Griechischen Tanne (Abies cephalonica), die hier in genetisch reiner Form vergesellschaftet mit Blaustern (Scilla bifolia subspec. nivalis), Griechischem Schaumkraut (Cardamine graeca), FingerLerchensporn (Corydalis solida), Balkan-Windröschen oder Blaue Anemone (Anemona blanda) und dem Doldenblütler (Geocaryum cynapioides $=$ Huetia cynapioides subspec. macrocarpa) vorkommt. In mittleren Höhenlagen ist die Fichte (Picea abies) dominant, während in unteren Lagen die Kermeseiche (Quercus coccifera) den Hauptbaumbestand bildet. Am Berg Roudi mit seinem höchsten Gipfel, dem Mount Yiouparie, im Nordwestbereich des Parkes ist die Macchi in typischer Art und Weise ausgebildet. Die im Nationalpark herrschende Tannenwaldzone (Abietion cephalonicae-Zone) ist die herausragende vegetationskundliche Besonderheit, die auch Gegenstand einer ausführlichen wissenschaftlichen Untersuchung war (KNAPP, 1965). Bedeutsam für Kefalonia ist, dass auf ihr endemische Pflanzentaxa, wie das Kefalonische Veilchen (Viola cephalonica), das Enos-Seifenkraut (Saponaria aenesia), Östliche Günsel (Ajuga orientalis subspec. aenesia) und Helmkraut (Scutellaria rubicuda subspec. cephalonica) sowie etliche Griechenlandendemiten wie z.B. Poa cephalonica vorkommen.

Die Säugetierfauna ist gut bekannt und weist mit einem Wildpferdvorkommen im Nationalpark eine Besonderheit auf. Weiterhin ist auch die Avi- sowie die Reptilien und Amphibienfauna gut erforscht (vergl. www.kefaloniathewaytogo.com, abgerufen am 22.09.2015). Von den Insekten gibt es nur wenige, verstreute Literaturangaben (z.B. PAPAPAVLOU \& KATSOUNI, 2008 zur Orthopterenfauna) und auch in der Köcherfliegenfauna Griechenlands gibt es nur einzelne Angaben (MALICKY, 2005). Der Kenntnisstand über die anderen Tiergruppen ist dagegen als völlig unzureichend einzuschätzen.
Bisher konnten im Nationalpark folgende Lepidopterenarten nachgewiesen werden: Der erste Nachweis einer Schmetterlingsart aus dem heutigen Nationalpark findet sich bei Rebel (1910: 425) für Pungeleria capreolaria (Denis \& SChiffermüller, 1775). Dann fand MAlicky (1992: 401) Catocala eutychea Treitschke, 1835 an den Osthängen des Enos.

Papapavlou \& Katsouni (2008) geben Pieris ergane (Geyer, 1828), Issoria lathonia (Linnaeus, 1758), Favonius quercus (LinnaEus, 1758), Hipparchia volgensis-Gruppe und Hyponephele cf. lupinus (O. CosTA, 1836) vom Enos und Roudi an.

In den Jahren 2001, 2002, 2003, 2005 und 2015 hat der Autor hauptsächlich am Tage an der Westflanke des Enos um 650 bis $1350 \mathrm{~m}$ und in der Gipfelregion bei $1600 \mathrm{~m} \mathrm{NN}$ gesammelt. Zwei Lichtfänge, d.h. einmal ambulant und einmal stationär, wurden dort durchgeführt und die Lichtfalle kam ebenfalls zum Einsatz. Beobachtet wurden Bankesia cephalonica spec. nov., Heliopsychidea graecella (Millière, 1866), Dyspessa ulula (BORKHAUSEN, 1790), Macroglossum stellatarum (Linnaeus, 1758), Pieris rapae (Linnaeus, 1758), Colias croceus (Geoffroy in Fourcroy, 1785), Lyceana phlaeas (Linnaeus, 1761), Issoria lathonia (Linnaeus, 1758), Vanessa atalanta (Linnaeus, 1758), Vanessa cardui (Linnaeus, 1758), Watsonalla uncinula (BoRkHAUSEN, 1790), Rhopotria asperaria (HÜBNER, 1817), Menophra abruptaria (THUNBERg \& SEBALDT, 1792), Peribatodes rhomboidaria (Denis \& SChIFFERMülleR, 1775), Peribatodes umbraria (HüBner, 1809), Cyclophora puppillaria (HüBNER, 1799), Idaea filicata (HüBNER, 1799), Idaea degeneraria (HüBNER, 1799), Coenotephria ablutaria (BoIsDuval, 1840), Thera variata (DENIS \& SCHIFFERMÜller, 1775), Thera britannica (Turner, 1925), Drymonia ruficornis (Hufnagel, 1766), Catocala eutychea TreitschKe, 1835, Ophiusa tirhaca (Cramer, 1773), Zethes insularis Rambur, 1833, Eutelia adulatrix (HÜBNER, 1809-1813), Caradrina clavipalpis (Scopoli, 1763), Apamea sicula (Turati, 1909), Agrotis puta (HüBner, 1803) und Arctia festiva (Hufnagel, 1766). Somit sind im Nationalparkgebiet bisher 35 Arten nachgewiesen.

\section{Spezieller Teil}

\subsection{Allgemeines}

Für die Beschreibung der einzelnen Fundnachweise werden aus Platzgründen verschiedene Wertigkeiten gesetzt. So wird bei den allgemein häufigen und verbreiteten Arten auf detaillierte Angaben verzichtet und diese nur in der Tab. 1 aufgeführt. Bei nicht allzu häufigen Arten werden neben den Fundorten zusätzlich Angaben zur Phänologie und lediglich bei faunistisch interessanten Arten weitergehende Informationen bzw. Kommentare gegeben. 
Für den speziellen Teil werden folgende, gebräuchliche Abkürzungen verwendet:

$\begin{array}{ll}\text { cult. } & \text { kultiviert } \\ \text { e.o. } & \text { ex ovo } \\ \text { e.l. } & \text { ex larva } \\ \text { e.p. } & \text { ex pupa } \\ \text { Ex. } & \text { Exemplar } \\ \text { FL. } & \text { Lichtfalle } \\ \text { Gen.det. } & \text { Genitaldetermination } \\ \text { LF. } & \text { Lichtfang } \\ \text { i.A. } & \text { in Anzahl (5 bis 9 Ex.) } \\ \text { i.M. } & \text { in Menge (ab 10 Ex.) } \\ \text { in litt. } & \text { brieflich mitgeteilt } \\ \text { oberh. } & \text { oberhalb } \\ \text { OESZ } & \text { Osteuropäische Sommerzeit } \\ \text { W, N, E, S } & \text { Himmelsrichtungen } \\ \text { Umg. } & \text { Umgebung }\end{array}$

\subsection{Fundortverzeichnis}

Nachfolgend wird die Darstellung der eigenen Fundorte gegeben. Im Text werden nur die vorangestellten Kurzformen der Fundorte verwendet. Die Bezeichnung der Lokalitäten wurde wesentlich der Karte „Kefalonia \& Ithaka" vom Verlag "tc travel maps“, Maßstab 1:80000, ISBN 3-933074-03-07 entnommen.

$\begin{array}{ll}\text { Lefkada } & \\ \text { Elithea } & \text { Umg. Elithea 1 km S, 600 m NN } \\ \text { Lefkada } & \text { S der Ortschaft, } 5 \mathrm{~m} \mathrm{NN} \\ \text { Kamilio } & \text { Umg. Kamilio S Kirche, } 500 \mathrm{~m} \mathrm{NN} \\ \text { Katohori } & \text { E der Ortschaft, } 150 \mathrm{~m} \mathrm{NN} \\ \text { Nikiana } & \text { N der Ortschaft, } 1 \mathrm{~m} \mathrm{NN} \\ \text { Vliko } & \text { SW der Ortschaft, } 50 \mathrm{~m} \mathrm{NN}\end{array}$

Ithaka

Anogi

Exogi

Frikes

Kalamos-

Quelle

Kioni

Lefki

Marathias

Monastery

Taxiarchon

Ormos

Kaminia
Piso Aetos Hafenanlage und Umg., 5-20 m NN

Platrithias Umg. $1 \mathrm{~km} \mathrm{~N}, 50 \mathrm{~m} \mathrm{NN}$

Stavros Ortschaft und Umg. S, 100-120 m NN

Vathi Ortschaft und $\mathrm{N}$ anschließende Maccie, 5-10 m NN

\section{$\underline{\text { Kefalonia }}$}

Agios Efimia Umg. SE, $5 \mathrm{~m} \mathrm{NN}$

Agios Nationalpark „Enos“, Umg. Agios Eleft-

Eleftherois herios NE, 650-700 $\mathrm{m} \mathrm{NN}$

Agon ca. $1 \mathrm{~km} \mathrm{NNE} \mathrm{der} \mathrm{Ortschaft,} 250 \mathrm{~m} \mathrm{NN}$

Argostoli nördliche Umg. von Argostoli N, 5-50 m NN

Assos Ortschaft und Umg. SE, 0-200 m NN

Aviathos lake Umg. Agios Nikolaos NW, 300 m NN

Cioupali NW - Hänge des Berges, 500 m NN

Defaranata Ortschaft und Umg. S, $350 \mathrm{~m} \mathrm{NN}$

Enos Nationalpark „Enos“, Gipfelbereich, $1.600 \mathrm{~m} \mathrm{NN}$

Konidarata Ortschaft N, $250 \mathrm{~m} \mathrm{NN}$

Kourouklata Ortschaft und Umg. S, 150 m NN

Livadi ca. 2,5 km NNE der Ortschaft, $50 \mathrm{~m} \mathrm{NN}$

Magganos Ortschaft S, $200 \mathrm{~m} \mathrm{NN}$

Monastery Kloster bei Poros, $200 \mathrm{~m} \mathrm{NN}$

Theotokou

Atrou

Monastiráki Umg. Monastiráki W bei Agios Nikolaos, $200 \mathrm{~m} \mathrm{NN}$

Myrto Bay oberhalb der Myrto Bay an den Westhängen des Skoloupas, $300 \mathrm{~m} \mathrm{NN}$

Ormos Bucht NE von Plagia, 0-10 m NN

Kakogilos

Patrikata

Ortschaft und Umg. NW, 400-550 m NN

Poros Ortschaft und Umg, $60 \mathrm{~m} \mathrm{NN}$

Razata Ortschaft $2 \mathrm{~km} \mathrm{E,} 430 \mathrm{~m} \mathrm{NN}$

Sami Ortschaft und nähere Umg., 0-5 m NN

Valsamata Ortschaft NE, $550 \mathrm{~m} \mathrm{NN}$

\subsection{Systematisches Verzeichnis (Lepidoptera)}

In der Tab. 1 sind alle Arten und ihre Nachweise für die einzelnen Inseln dargestellt. Nachfolgend wird auf spezielle Arten eingegangen, die erstmals beschrieben werden, Erstfunde für die Ionischen Inseln darstellen bzw. auch in faunistischer und phaenologischer Hinsicht interessant sind. Im Wesentlichen wird der Systematik und Reihenfolge von Karsholt \& RAzowsKi (1996) gefolgt. 
Hepialidae

Triodia adriaticus OSTHELDER, 1931

Neu für die Ionischen Inseln.

DAHL fand 2 Ex. am 24.09.2007 am Licht bei Roda, Robolla Beach (Korfu) (in litt. 2016; vergl. Foto unter www.lepiforum.de).

\section{Tineidae}

\section{Nemapogon scholzi SUTTER, 2000}

Die Art wurde von Zakynthos, südlich Vasilikos nach $40^{\top} o^{T}$ und einem +9 vom September 1997 und einem einem $\sigma^{\text {t }}$ von Kreta, Makrigialos vom Juni 1998 beschrieben. Alle Tiere wurden am Licht gefangen. Neuere Funde sind offenbar nicht bekannt.

\section{Psychidae}

In der Systematik und Reihenfolge wird an dieser Stelle ARNSCHEID \& WeIdLICH (2016) gefolgt.

\section{Eumasia parietariella (HEYDENREICH, 1851)}

Rebel (1910: 431) beschreibt ein Einzelstück von Kefalonia, welches auch bei GozMANY (1983: 255) erwähnt wird. Die Raupen in ihren typischen Säcken konnten auf den Inseln Kefalonia, Ithaka, und Lefkada an mehreren Stellen, teilweise i.A., nachgewiesen worden: Valsamata, Myrto Bay, Cioupali, Patrikata, Kourouklata, Konidarata, Magganos, Assos, Livadi, Argostoli, Anogi, KalamosQuelle, Platrithias, Stavros, Lefki, Frikes, Monastery Taxiarchon, Exogi, Vathi, Kioni, Piso Aetos, Lefkada, Vliko und Katohori. Die Imagines schlüpften zwischen dem 03.05. und Anfang Juni.

\section{Bankesia cephalonica spec. nov.}

Derivatio nominis: Die neue Art wird nach der Insel Kefalonia benannt, auf der sie offenbar endemisch vorkommt.

Holotypus: $0^{\top}$, 02.05.2003 (Flügelspanne 13,0 $\mathrm{mm}$. Fig. 1): Europa meridionalis, Greece-West/Kefalonia, Ionische Inseln, Nationalpark Enos-Gipfel, $1600 \mathrm{~m} \mathrm{NN}$, leg. Dr. M. Weiduich. Er befindet sich in coll. Museum für Naturkunde des Leibniz Institutes in Berlin.

Paratypen (die gezüchteten Exemplare jeweils mit Sack und Puppenhülle): Männchen: $1 \sigma^{\top}$ 01.05.2001, $1 \sigma^{\top}$ e.p. 06.05.2003 jeweils mit Sack und Puppenhülle, 17 o $0^{\circ}$

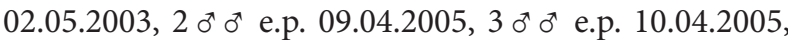

$2 \sigma^{\pi} \sigma^{\top}$ e.p. $11.04 .2005,1 \sigma^{\top}$ e.p. $14.04 .2005,4 \sigma^{\top}$ e.o. 25.01.2016: Europa meridionalis, Greece-West/Kefalonia, Ionische Inseln, Nationalpark Enos-Gipfel, 1600 m NN.

Weibchen: 1 o 01.05.2003, 1 ㅇ e.p. 01.05.2003, 1 क e.p. 04.05.2003, 3 ㅇ ㅇ e.p. $05.05 .2003,5$ q o e.p. 06.05.2003, 1 ㅇ e.p. $07.05 .2003,4$ 우 e.p. 11.04.2005, 2 우 e.p. 12.04.2005, 3 우 우 e.p. $13.04 .2005,1$ 우 e.p. $14.04 .2005,1$ 우 e.p. 15.04.2005, 2 우 e.p. 16.04.2005, jeweils mit Sack und Puppenhülle, 2 ㅇ o e.o. 25.01.2016, 1 ㅇ 27.01.2016: Europa meridionalis, Greece-West/Kefalonia, Ionische Inseln, Nationalpark Enos-Gipfel, 1600 m NN.

Säcke: 75 Säcke 30.04.-01.05.2001, darunter $12 \mathrm{mit}$ + Puppenhülle; 29 Säcke 14.-15.05.2002 darunter 1 mit $\sigma^{7}$ Puppenhülle und 4 mit $q$ Puppenhülle; 82 Säcke 01.05.2003 darunter 2 mit + Puppenhülle; 39 Säcke 05.05.2015 darunter 3 mit $0^{\top}$ Puppenhülle und 20 mit + Puppenhülle, 68 Säcke e.o. Januar 2016: Europa meridionalis, Greece-West/Kefalonia, Ionische Inseln, Nationalpark Enos-Gipfel, $1600 \mathrm{~m} \mathrm{NN}$, alles leg. Dr. M. WeIDLICH.

Das Typenmaterial umfasst somit $32 \sigma^{\pi} \sigma^{\top}$ und 28 o $\circ$, die gezüchteten Ex. jeweils mit Sack und Puppenhülle sowie 293 Säcke. Es befindet sich in den Sammlungen W. R. ARNSCHEID (Bochum/Deutschland), Naturkundemuseum der Leibniz Gesellschaft (Berlin/Deutschland), Tiroler Landesmuseen-Betriebsgesellschaft m.b.H. (Innsbruck/Österreich) und Z. ToKÁR (Michalovce/ Slowakei).

Diagnose: Männchen: Mittelgroße Falter mit einer Flügelspanne von 11,7 bis $13,4 \mathrm{~mm}$. Augen schwarz, rund und der Augenindex (Quotient aus Augenabstand bis maximalen Augendurchmesser) beträgt etwa das Doppelte des Augendurchmessers. Ocellen vorhanden, die Stirnschopfbehaarung relativ lang, länger als der Augendurchmesser, bestehend aus gelbbräunlichen Härchen, Labialpalpen sehr lang und dreigliedrig.

Die Fühlerlänge erreicht nicht ganz die Länge des Vorderflügelcostalrandes. Fühlergliederzahl 29-32, Basalfühlerglieder dicht bräunlichweiß beschuppt, Fühlerglieder leicht beschuppt und ventral quirlig lang behaart, wobei die Länge der Behaarung etwa das 1,5 fache der Fühlergliedlänge ausmacht (Bereich 15. bis 20. Fühlerglied). Körper ebenfalls bräunlich behaart, letztes Körpersegment etwas heller, gelblichbraun.

Vorderflügel schmal und langgestreckt, Färbung gelblich mit unterschiedlichen, unregelmäßig ausgebildeten bräunlichen Flecken, insgesamt matt golden schimmernd. Diskoidalfleck nur schwach ausgeprägt. Deckschuppen breit; vier- und fünfzackig (Klasse IV-V nach SAUTER, 1956). Vorderflügel mit Anhangszelle, ohne Eingeschobene Zelle, 10 Diskoidalzelladern, die alle, bis auf $\mathrm{r} 4$ und $\mathrm{r} 5$, welche sehr lang gestielt sind, getrennt voneinander entspringen. Bei einem Exemplar tritt $\mathrm{r} 2$ und r3 kurz gestiehlt auf. Fransen gescheckt, etwas 
heller als die Flügelfärbung, Fransenschuppen ein- bis dreizackig, lancettähnlich.

Hinterflügel einfarbig hell bräunlichgrau, Fransen etwas heller, am Apikalrand deutlich dunkler. Sie sind sehr lang und bestehen hauptsächlich aus dreizackigen, lancettähnlichen Schuppen. Die Hinterflügeladerung mit 6 Diskoidalzelladern, die alle getrennt voneinander entspringen.

Vordertibie mit langer Epiphyse, die fast 1/3 der Länge der Vordertibie erreicht und unter einem kräftigen Haarbüschel angeordnet ist. Mitteltibie mit einem, Hintertibie mit 2 Spornpaaren, alle Beine mit 5 Tarsengliedern.

Genitalarmatur dorso-ventral flach ausgebildet, Valven lang und nicht sehr schmal, Sacculus mit Clasper kurz und breit, anal abgerundet. Tegumendach deutlich eingekerbt, Vinculum breit und „V“-förmig, ohne Saccus. Phallus lang, deutlich gebogen und ohne Cornuti (Fig. 2).

Weibchen: Flügellos, weißgelb bis hellbräunlich, Körperlänge 3,9-4,1 mm in leicht gekrümmter, natürlicher Haltung (ohne Ovipositor), -durchmesser etwa 1,0-1,2 mm. Augen klein schwarz und rund, keineOcellen, 12-14 Fühlerglieder, Tarsen dreigliedrig. Körperform leicht gekrümmt, schüttere Körperbehaarung, Afterwollhaare silbrig und kranzförmig am 7. Segment vorhanden (Fig. 3).

Sack: Säcke in den Abmaßen sehr unterschiedlich, ein ausgeprägter Geschlechtsdimorphismus ist nicht immer $\mathrm{zu}$ erkennen, wenngleich die $\sigma^{\star} \sigma^{\star}$-Säcke meisten kleiner sind: Länge 7 bis $10,4 \mathrm{~mm}$ Breite 2 bis $3 \mathrm{~mm}$, Höhe 2,5 bis $3 \mathrm{~mm}$ (adulte Säcke von geschlüpften Imagines) (Fig. 9 a, e). Der Sack ist relativ einheitlich und sehr deutlich dreikantig ausgebildet, die Färbung ist dunkel- bis schwarzbraun. Detritus, Chitinteile, kleine Mollusken und verschiedene Reste von Abies cephalonica charakterisieren den Sack weiterhin.

Differentialdiagnose: Von der Gattung Bankesia TutT, 1899 wurden bisher vier Arten beschrieben: conspurcatella (Zeller, 1850), montanella (WALsingham, 1899), desplatsella Nel, 1999 und pallida (STAudinger, 1879). B. conspurcatella kommt in Westeuropa, hauptsächlich im mediterranen Raum, aber auch im altlantischen und subatlantischen Bereich Mitteleuropas nördlich bis nach Dänemark vor. $B$. desplatsella wurde aus Frankreich bekannt, $B$. montanella existiert endemisch auf Korsika und B. pallida ist nach $3 \sigma^{*} \sigma^{*}$ aus Amasia (heute Amasyi, in der Türkei) als eine Art von „Talaeporia“ beschrieben worden (Staudinger, 1879: 268). Rebel (1901: 229) transferiert diese Art in die Gattung Bankesia. Hierzu fehlen neuere Untersuchungen und es ist unklar, ob dieses aufrecht erhalten werden kann.

Die zoogeographisch der neuen Art am nächsten verbreitet ist B. conspurcatella in Calabrien (Italien) (vergl. WeIDLICH, 2015: 1921). Im Habitus ähnelt die neue Art der B. conspurcatella wie auch B. desplatsella, wenngleich diese im Durchschnitt kleiner sind. Unter- schiede gibt es aber in der Vorderflügelform, diese ist bei bei $B$. conspurcatella und $B$. montanella schmaler und sodurch gestreckter wirkend. B. montanella ist weiterhin durch eine weißliche-silbrige Färbung leicht von den beiden anderen Arten zu unterscheiden. Wenngleich die Schuppenklassen der Vorderflügeldeckschuppen identisch sind, ist im Gesamtbild der Anteil der schmaleren Schuppen bei B. cephalonica spec. nov. deutlich höher als bei den beiden anderen Arten.

Auch im Genitalbau lassen sich deutliche Unterschiede finden: Die Valven sind nicht so lang und schmal wie bei B. conspurcatella, B. desplatsella und B. montanella, sondern relativ kurz und kräftig. Der Clasper des Sacculus ist nicht lang und spitz ausgezogen wie bei den verwandten Arten, sondern kurz und kräftig sowie mit Cornuti versehen. Auch der Phallus zeigt eine andere Form, er ist bei der neuen Art deutlich gebogen im Vergleich zur gestreckten, schwach gebogenen Form bei den anderen Bankesia-Arten.

Die Weibchen unterscheiden sich hauptsächlich in der Fühlergliederzahl, B. consurcatella weist 5 bis 6 Glieder auf, B. montanella nur 3 bis 4 Glieder und B. cephalonica spec. nov. verfügt über lange Fühler mit 12-14 Gliedern (inclusive Scapus und Pedicellus). Verschmelzungen der Tarsenglieder auf 2 Segmente, wie sie bei B. conspurcatella und B. montanella des Öfteren zu beobachten ist, wurden bei B. cephalonica spec. nov. bisher nicht beobachtet. Die Weibchen von pallida und desplatsella sind bisher unbekannt.

Weiterhin zeigen auch die Säcke gewisse Unterschiede auf. Diejenigen von B. cephalonica spec. nov. sind im Durchschnitt größer und haben eine dunklere Färbung als die der anderen Bankesia-Arten.

Die morphologischen Differenzen werden unterstützt durch die vorliegenden genetischen Daten. Die DNA-Isolation und DNA-Sequenzierung erfolgten am CCanadian Centre for DNA Barcoding, Ontario, Canada nach den bei DEWAARD et al. (2008) beschriebenen Standardprotokollen. Mit Hilfe dieser Methoden wurde eine 658 Basenpaare umfassende Region der mitochondrialen Cytochrom C Oxidase I (COI, Barcodefragment 5, 658bp) einschließlich der 648 Basenpaare des Barcodes isoliert und vervielfältigt und der Berechnung paarweiser genetischer Distanzen mit Hilfe des Kimura 2 Parameter Modells (K2P) unterzogen (RATNASINGHAM \& HeBERT, 2013).

In den Jahren 2013, 2014 und 2015 wurden DNASequenzen von verschiedenen Psychiden-Taxa, u.a. im Rahmen des iBOL-Projekts „Psychidae of East- and South-Europe - POESE, untersucht. Darunter befanden sich B. cephalonica spec. nov. und die weiter unten beschriebenen Reisseronia-Taxa. Von B. cephalonica spec. nov. wurde ein Paratypus ơ, e.p. 14.04.2005: Europa meridionalis, Greece-West/Kefalonia, Ionische Inseln, Nationalpark Enos-Gipfel, $1600 \mathrm{~m} \mathrm{NN}$, leg. Dr. M. WeIDLICH untersucht. Das Ergebnis wurde mit den vorliegenden, zugänglichen Daten von B. conspurcatella und B. montanella verglichen. So zeigt die neue Art 
deutliche Differenzen und weist Unterschiede von 6-7 \% $\mathrm{zu}$ B. conspurcatella sogar $27 \% \mathrm{zu}$ B. montanella auf.

Die im nicht weit entfernten Epirus (Zagoria) vorkommende Pseudobankesia lichenaria WeIDLICH, 2016 unterscheidet sich von B. cephalonica nov. spec. um $36 \%$ (vergl. Tab. 2 und Fig. 4). Die Ergebnisse belegen, dass es sich hier um eine für die Wissenschaft neue Art handelt.

\section{Die Entdeckung der neuen Art und Angaben zur Ökologie und Biologie:}

Wer mit der Fähre von der Peloponnes (z.B. Killini oder Patras) nach Argostoli kommt, fährt an der Südküste von Kefalonia entlang. Steil und imposant erhebt sich der Enos aus dem Ionischen Meer. Insbesondere bei schönem Wetter bietet dieses Gebirgsmassiv einen überwältigen Eindruck. Erstmalig war der Autor auf dem Massiv am 30.04.2001 und der Blick reichte bei herrlichem Wetter und klarer Sicht bis zur südöstlich vorgelagerten Insel Zakynthos. Nachdem im Gipfelbereich vorerst ergebnislos die Felsen (anstehender Dolomit) abgesucht wurden, konnten dann unter Steinen die ersten Säcke der neuen Bankesia gefunden werden. Insgesamt wurden an diesem und am folgenden Tage über 70 Säcke gesammelt, darunter etliche mit geschlüpften, leeren weiblichen Puppenhüllen. Ein totes Männchen befand sich nahe der Bodenvegetation in einem Spinnennetz. Auch im nachfolgenden Jahr, so am 14.05., wurde wieder der Gipfel besucht. Zu diesem Zeitpunkt war die Imaginalphase erwartungsgemäß wie auch schon im vorigen Jahr vorbei und es konnten nur wenige leere Säcke gefunden werden. In die Planung für 2003 wurde abermals eine Exkursion zum Enos aufgenommen. Das Frühjahr gestaltete sich in diesem Jahr völlig anders. So fielen auf den Ionischen Inseln bedeutende Niederschläge. Infolgedessen war der Gipfel am 01.05.2003 noch weitestgehend mit Schnee bedeckt und die Geröllflur nur an wenigen Stellen zugänglich. Nach längerem Suchen konnte der Autor um 12.55 Uhr (OESZ) in den Geröllfluren unter Steinen ein Männchen sowie unmittelbar daneben ein frisch befruchtetes, eierlegendes 9 entdecken. Insgesamt wurden an diesem Tag etwa 80 Säcke gesammelt. Am nächsten Tag ging es in aller Frühe, noch in der Dunkelheit, abermals hinauf. Im ersten Sonnenlicht, kurz nach Sonnenaufgang, flogen etliche $0^{\top} o^{\top}$ dicht über die Geröllfelder von 06:45 bis 07:40 Uhr (OESZ). In den nächsten Tagen schlüpften weitere $0^{\top} o^{\top}$ und i $o$.

Am 06.04.2005 besuchte der Autor nunmehr das vierte Mal den Enos. Obwohl in diesem Jahr wesentlich weniger Schnee als 2003 hier oben lag, fanden sich etwa 30 Säcke, einer mit geschlüpfter of Puppenhülle. Von fliegenden $\sigma^{\top} \sigma^{\top}$ war keine Spur zu sehen.

10 Jahre später war der Autor abermals in der Gipfelregion, so am 05.05.2015 (Fig. 12). Die Straße hinauf, die vormals ein unbefestigter Weg war, wurde mit EU-Fördermitteln zu einer modernen Asphaltstraße ausgebaut, Kosten 3 Millionen Euro. Hier oben waren um
6 Uhr (OESZ) 13 Grad und es flogen Abertausende Tipuliden. Die Flugzeit der Bankesia war jedoch in diesem Jahr auch schon vorbei, denn unter den 39 gefundenen Säcken befanden sich 3 geschlüpfte Säcke mit ơ ơ und 20 mit i + Puppenhüllen.

Der Schlupf der Räupchen der nachfolgenden Generation vollzog sich vom 20. bis zum 22.05.2015. In einem Terrarium verlief die Zucht recht problemlos und im Spätherbst waren die Raupen erwachsen. Die Überwinterung erwies sich sich ziemlich schwierig und aus dieser Eizucht schlüpften im Januar 2016 nur wenige Imagines. Als Lebensraum ist bisher nur die Gipfelregion des Enos bekannt geworden. Dort leben die Raupen von Bankesia cephalonica spec. nov. sehr versteckt in verschiedenen Hohlräumen unter Steinen der in südliche Richtung geneigten, großenteils offenen Geröllhalden in Höhen um $1600 \mathrm{~m}$ NN. Sie ernähren sich offenbar nur von niederen Pflanzen, wie Algen, Flechten auch Moosen sowie von Detritus und verlassen den unmittelbaren Larvalhabitat nicht. Hier spinnen sie sich zur Verpuppung an die Unterseiten der Steine an. Etwas häufiger wurden die Säcke an den Stellen angetroffen, wo die Gesteinsflur noch zusätzlich durch verschiedene, hier meist in Gruppen stehende Abies cephalonica-Bäume beschattet werden. Das dort deutlich feuchtere Mikroklima lässt einen stärkeren Moosbewuchs an den Steinen zu.

Die Imagines sind im Freiland zwischen dem 01. und 02.05. beobachtet wurden. Bei der Zucht, ausser bei der Eizucht, schlüpften die Männchen vom 09.04 bis zum 06.05. und die Weibchen im Zeitraum vom 11.04. bis 07.05. Die Phänologie ist augenscheinlich sehr witterungsabhängig und die Imagines erscheinen bereits unmittelbar nach der Schneeschmelze. Bei geringen Niederschlägen im Winter und entsprechend höheren Temperaturen im Frühjahr ist es durchaus möglich, dass die ersten Tiere bereits Ende März schlüpfen. Die Eiablage erfolgt in den Sack und die Art ist univoltin.

Vergesellschaftet ist B. cephalonica spec. nov. mit Heliopsychidea graecella (MILLIÈre, 1866).

\section{Typhonia spec.}

\section{Neu für die Ionischen Inseln.}

Nur auf Kefalonia in der Umg. von Argostoli gefunden: 05.05.2015 zwei Raupen östlich von Razata. Von der Peloponnes ist T. christenseni (HätTENSCHWILER, 1990) bekannt.

\section{Penestoglossa dardoinella (MıLLIÈRE, 1863)}

Neu für die Ionischen Inseln.

An wenigen Stellen auf Ithaka und Kefalonia nachgewiesen: Frikes: ein Sack am 27.04.2000; Stavros: 11 Säcke vom 03.-07.05.2001, Schlupf je 1 ㅇ am 23. und 25.08.2001, 12 Säcke am 10.05.2002; Vathi: 1 Sack am 05.05.2001; Perahori: 4 Säcke am 11.05.2002, Schlupf von 
$20^{*}$ und 2 i zwischen dem 14.08. und Anfang 09.2002; Magganos: Eine Raupe am 14.05.2002, am 04.08.2002 schlüpfte ein + ; Lefki ein Sack ein Sack am 03.05.2003. und Myrto Bay ein leerer Sack am 06.04.2005.

\section{Luffia cf. lapidella (GOEZE, 1783)}

Bei Argostoli (Kefalonia) mehrere leere Säcke von BEIER gefunden (ReBEL, 1932: 55). Vom Autor auf Ithaka ab 1993 an der Kalamos-Quelle, Frikes, Exogi und Lefki und auf Kefalonia nahe der Myrto Bay und Konidarata nachgewiesen (auschließlich Sackfunde).

\section{Psyche crassiorella (BRUAND, 1850)}

\section{Neu für die Ionischen Inseln.}

Die Säcke mehrfach i.A. bei Lefkada, Nikiana, Vliko und Katohori ab 2000. Daraus schlüpften ein ơ am 18.05.2003 und 2 우 우 am 07.05.2015 (Katohori) sowie ein $\sigma^{*}$ am 06.05.2005 und ein ㅇ am 21.05.2012 (Vliko).

\section{Peleponnesia glaphyrella (REBEL, 1906)}

Rebel (1932: 55, 1938: 79) vermerkt Funde von Lefkada: $1 \sigma^{\top}$ am 17.04.1929, leg. Beter bei Enkluvi und 2 leere Säcke von Spartilla.

Ab 2001 wurden die Säcke relativ verbreitet und stellenweise auch häufig auf Kefalonia (Agios Efimia, Myrto Bay, Komitata, Argostoli, Konidarata, Poros, Magganos, Monastery Theotokou Atrou, Kourouklata, Agon) und Ithaka (Stavros, Lefki, Marathias, Vathi) gefunden. Die $\sigma^{\top} \sigma^{\top}$ fliegen im ersten Morgenlicht, so am 01.05.2003 ein Ex. um 7.05 Uhr am Aviathos lake und am 07.04.2005 an der Ormos Kakogilos 6 Ex. zwischen 07:35 und 08:00 Uhr (OESZ). Sie stellen mit dem ersten Sonnenstrahlen ihre Flugtätigkeit ein. Bei der Zucht schlüpften die Imagines zwischen dem 13.04. und 04.05.

\section{Reisseronia ionica spec. nov.}

Die Entdeckung der neuen Art: Bereits im Jahr 1993, beim ersten Besuch von Ithaka mit der Familie, fielen uns einige Säcke auf, die an Proutia betulina (Zeller, 1839) und Psyche crassiorella (BRUAND, 1850) erinnerten. Diese befanden sich angesponnen an Hauswänden und wurden als Belege eingesammelt. Daraus schlüpften aber keine Imagines mehr, denn es war bereits Mitte Juli gewesen. Erst 7 Jahre später und in den Folgejahren darauf wurde umfangreiches Material eingetragen und Imagines gezüchtet.

Die Nominatform wurde beim nächsten Besuch der Inselgruppe im Jahre 2001 auf Kefalonia entdeckt. Am 02.05.2001 wurden einige wenige Säcke bei Patrikata und Agon, ebenfalls an Felsen angesponnen, gefunden. Ein Jahr später befanden sich am 08.05.2002 auf Lekada an den Felswänden bei Vliko eine große Zahl an Reisseronia-Säcken, unter denen vier geschlüpfte $\sigma^{\top} \sigma^{\star}$-Säcke mit Puppenhüllen waren.

Derivatio nominis: Die neue Art wird nach der Inselgruppe, den Ionischen Inseln benannt, auf der sie offenbar endemisch vorkommt.

Holotypus: $\sigma^{\top}$, Europa meridionalis, Greece-West/Kefalonia, Ionische Inseln, Skoloupas, Myrto Bay, 300 m NN, e.l. 21.05.2005, leg. Dr. M. Weidlich (Flügelspanne $12,0 \mathrm{~mm}$ ) (Fig. 5 a). Er befindet sich in coll. Museum für Naturkunde des Leibniz Institutes in Berlin.

Paratypen (die gezüchteten Exemplare jeweils mit Sack und Puppenhülle): Männchen: $4 \sigma^{x} \sigma^{x}, 14.05$. und $28 \sigma^{x} \sigma^{x}$, 16.05.2002 (Anflug); $28 \sigma^{\star} \sigma^{\star}$, e.p., 15.05.-12.06.2002;

$12 \sigma^{\top} \sigma^{\star}$, e.l./e.p. 06.05.-02.06.2003; $81 \sigma^{\star} \sigma^{\star}$, e.1. 30.04.-Anf. 06.2005: Europa meridionalis, Greece-West/Kefalonia, Ionische Inseln, Skoloupas, Myrto Bay, 300 m NN; $4 \sigma^{\top} \sigma^{\top}$, e.l./e.p. 06.-30.05.2015: Europa meridionalis, GreeceWest/Kefalonia, Ionische Inseln, Umg. Livadi $2 \mathrm{~km} \mathrm{~N}$, $150 \mathrm{~m} \mathrm{NN}$;

4 ơ ơ, e.p. 14.05.2015, Assos, ca. $1 \mathrm{~km} \mathrm{E,} 200 \mathrm{~m} \mathrm{NN}$, alles leg. Dr. M. WEIDLICH.

Weibchen: 11 ㅇ ㅇ: e.l und e.p. 16.05 bis Anf.07.2002; 17 ㅇ ㅇ: e.l und e.p. 08.05 bis Ende 06.2005: Europa meridionalis, Greece-West/Kefalonia, Ionische Inseln, Skoloupas, Myrto Bay, 300 m NN; 5 ㅇ : e.l./e.p., 20.05.01.06.2015 Europa meridionalis, Greece-West/Kefalonia, Ionische Inseln, Umg. Livadi $2 \mathrm{~km} \mathrm{~N}, 150 \mathrm{~m} \mathrm{NN}$, alles leg. Dr. M. WeIDLICH.

Säcke: 3 Säcke, 02.05.2001: Europa meridionalis, GreeceWest/Kefalonia, Ionische Inseln, $1 \mathrm{~km} \mathrm{NW}$ Patrikata, 400 m NN; 2 Säcke, 02.05.2001, 6 Säcke, 06.04.2005: Europa meridionalis, Greece-West/Kefalonia, Ionische Inseln, $1 \mathrm{~km} \mathrm{NNE} \mathrm{Agon,} 200 \mathrm{~m} \mathrm{NN;} 16$ Säcke, 13.05.2002, 11 Säcke, 04.05.2015, Assos, ca. 1 km E, 150-200 m NN; 227 Säcke 14. bis 16.05.2002; 68 Säcke 01.05.2003, 165 Säcke, 06.04.2005: Europa meridionalis, Greece-West/Kefalonia, Ionische Inseln, Skoloupas, Myrto Bay, $300 \mathrm{~m} \mathrm{NN}$; 1 Sack, 06.04.2005, Argostoli, $10 \mathrm{~m} \mathrm{NN}$; 21 Säcke 04.05.2015, Europa meridionalis, Greece-West/Kefalonia, Ionische Inseln, Umg. Livadi 2 km N, 150 m NN; 10 Säcke, 04.05.2015, Umg. Defaranata S, $350 \mathrm{~m} \mathrm{NN}$, alles leg. Dr. M. WeIDLICH.

Das Typenmaterial umfasst somit $162 \sigma^{\top} o^{\top}$ und 33 우, die gezüchteten Ex. jeweils mit Sack und Puppenhülle sowie 530 Säcke. Es befindet sich in den Sammlungen W. R. Arnscheid (Bochum/Deutschland), Naturkundemuseum der Leibniz Gesellschaft (Berlin/Deutschland), Senckenberg Gesellschaft für Naturforschung (Müncheberg/Deutschland), Tiroler Landesmuseen-Betriebsgesellschaft m.b.H. (Innsbruck/ Österreich) und Z. TokÁr (Michalovce/Slowakei). 
Diagnose: Männchen: Mittelgroße Falter mit einer Flügelspanne von 10,6 bis 13,3 mm, Färbung einfarbig schwarzbraun. Augen schwarz, rund und der Augenindex beträgt 1,0 bis 1,1 . Nebenaugen (Ocellen) nicht vorhanden. Kopfbehaarung schwarzbraun, Labialpalpen kurz und rudimentär. Die Fühlerlänge relativ kurz, sie erreicht die Hälfte der Länge des Vorderflügelcostalrandes. Fühlergliederzahl 21-23, Fühlerglieder dicht beschuppt, Kammzähne mit Wimpern versehen, deren Länge etwa der Kammzahnbreite entspricht. Die Kammzähne erreichen fast das Vierfache der Länge der Fühlerglieder (mittlerer Fühlerbereich).

Vorderflügel breit, Färbung einfarbig schwarzbraun, Diskoidalfleck nicht erkennbar. Deckschuppen schmal: ein- bis dreizackig, die dreizackigen Schuppen selten (Klasse I-II nach SAUTER, 1956). Vorderflügel mit EingeschobenerZelle, ohne Anhangszelle, 8 Diskoidalzelladern, die alle, getrennt voneinander der Diskoidalzelle entspringen. Fransen einfarbig schwarzbraun, leicht kupfrig schimmernd, lancettförmig aber breit, entspricht der Klasse 2 c nach Hauser (2012: 200).

Hinterflügelfärbung wie die der Vorderflügel, 5 Diskoidalzelladern, die alle getrennt voneinander entspringen. Hinterflügel mit haar- und lancettförmigen, zweizackigen und selten dreizackigen Schuppen bedeckt. Fransenschuppen lancettförmig, aber schmaler als die der Vorderflügelfransen. Körper schwarzbraun behaart. Vordertibie ohne Epiphyse, Mitteltibie und Hintertibie mit jeweils einem Spornpaar, alle Beine mit 5 Tarsengliedern.

Genitalarmatur Reisseronia-typisch und von rundlicher Gestalt.

Valven überragen das Tegumendach, welches zweifach leicht eingekerbt ist. Clavus deutlich ausgebildet mit Cornuti. Vinculum breit und rundlich gestaltet ohne Saccus. Anelli poyramidenförmig, Phallus gerade, kräftig ohne Cornuti (Fig. 8 a, d).

Weibchen: Flügellos, weiß bis hellbräunlich, auch rötlichbraun, Körperlänge 6-7,2 mm in gestreckter, natürlicher Haltung (ohne Ovipositor), -durchmesser etwa $2 \mathrm{~mm}$. Augen klein schwarz und rund, keine Ocellen, Fühler rudimentär, 2 bis 3 gliedrig, Tarsen dreigliedrig. Schüttere Körperbehaarung, Afterwollhaare silbrig und kranzförmig am 7. Segment vorhanden.

Sack: Säcke in den Abmaßen sehr unterschiedlich, ein Geschlechtsdimorphismus ist bei ihnen in den letzten Raupenstadien zu erkennen: Männchen: Länge 9-11 mm, Breite: 2,5-3,5 mm (Fig. 9 b); Weibchen: Länge 12-16 mm, Breite 3-4,5 mm (Fig. $9 \mathrm{f}$ ) (jeweils adulte Säcke von geschlüpften Imagines ohne überstehendes Baumaterial gemessen). Der Sack ist sehr unregelmäßig aufgebaut und erinnert an den von Psyche- und Proutia-Arten. Er besteht hauptsächlich aus Grashalmen, Blattresten und untergeordnet aus Blütenund Samenteilchen. Die Färbung reicht von mittelgrau bis bräunlich.
Differentialdiagnose: Bisher wurden 14 Reisseronia-Arten beschrieben. Darauf entfallen auf Reisseronia Sieder, 1956 sensu stricto zehn und auf den Subgenus Tsikalasia Hauser, 1996 vier Arten. Reisseronia s. str. zeichnen sich durch das Fehlen einer Epiphyse an den Tibien der $0^{x} \sigma^{x}$, die bei den Arten von Tsikalasia vorhanden ist.

Bei R. ionica spec. nov. sind keine derartigen Epiphysen ausgebildet und die Taxa R. gertrudae SiEDER, 1962 sowie R. imielinella Malkiewicz, Sobczyк \& LARYsz, 2013 sind parthenogenetisch. Im Vergleich müssen somit R. tarnierella (BRUAND, 1850), R. hofmanni (HeylaERTs, 1879), R. nigrociliella (REBEL, 1934), R. staudingeri (Heylaerts, 1879), R. pusilella (Rebel, 1940). R. magna HättensChWILER, 1982, R. arnscheidi WeIDlich, 2006 und die bisher nur vom asiatischen Teil der Türkei (Brussa) bekannt gewordenen $R$. flavociliella (MANN, 1864) diskutiert werden. Diese Arten, von denen die Säcke bekannt geworden sind, verfügen über eine einheitliche Form, bei der das eingebaute Material hauptsächlich aus Grasteilen besteht, die längs und enganliegend angeordnet sind. Im habituellen Vergleich werden deutliche Unterschiede in der Färbung zu R. staudingeri, welche silbergraue Vorderflügel aufweist, deutlich. Weitere Unterschiede zwischen R. tarnierella, R. flavociliella, R. hofmanni, R. nigrociliella, R. pusilela, R. magna (Fig. 4 c) und R. arnscheidi arbeiten HäTtensCHWILER (1982) und WEIDLICH (2006) heraus. Sie bestehen hauptsächlich in der Größe der Falter, der Anzahl der Antennenglieder bei $\sigma^{\star} \sigma^{\star}$ und $\circ$ o 9 und der Ausbildung der weiblichen Tarsenglieder. $R$. ionica spec. nov. unterscheidet sich von $R$. tarnierella- $\sigma^{*} \sigma^{*}$ wesentlich in der Flügelspanne (6-7 mm), in der Anzahl der Antennenglieder (16-18) sowie der Anzahl der 우 ㅇ Tarsenglieder (0, nur Krallen). R. nigrociella- $\sigma^{\top} \sigma^{\top}$ verfügen über kürzere Fühler mit nur 15-17 Gliedern und die ㅇ ㅇ über 4-5 Tarsalglieder.

Im Unterschied zu R. pusilella fallen wiederum die hier kurzen Fühler auf mit nur 15-17 Gliedern und die nicht so stark reduzierten Antennenglieder (5-6) bei den 우 우. Im Vergleich zu R. magna existieren die deutlichsten Unterschiede bei den Weibchen, die hier größer sind $(10 \mathrm{~mm})$ und über gut ausgebildete Beine mit 5 Tarsengliedern verfügen. Am ehestens ähneln die R. ionica spec. nov.-Merkmale denen von $R$. arnscheidi, von der sie aber durch die rundliche Vorderflügelform leicht differenziert werden kann.

Dazu ergänzt werden kann, dass sich alle ReisseroniaFalter über eine ausgeprägt rundliche Vorderflügelform definieren, bis auf die griechischen R. magna und R. mali$c k y i$, die über eine ähnliche Flügelform mit einem relativ spitzen Apex der Vorderflügel verfügen.

Auch in der bisher bekannten zoogeographischen Verbreitung differieren die diskutierten Arten deutlich. In Griechenland kommen nur R. nigrociliella, R. pusiellla (Festland) und R. magna (Peloponnes) und die R. Tsikalasia malickyi (endemisch auf Kreta) vor. 3 Arten unterscheiden sich aber auch durch ihre späte Flugzeit im Jahr von $R$. ionica spec. nov., die bei $R$. nigrociliella und erst im Juni und bei den anderen drei Arten erst im 
späten Juli beginnt. Lediglich bei $R$. pusilella beginnt die Flugzeit Ende April (vergl. WeidLich, 2008: 477).

Einer der gravierendsten Unterschiede lässt sich bei den Säcken feststellen. Wie bereits oben erwähnt, fallen die bisher bekannten Reisseronia-Säcke durch eine sehr einheitliche und vergleichbare Form auf. Die von $R$. ionica spec. nov. sind völlig anders strukturiert und ähneln sehr den Säcken von Proutia betulina ( $\left.o^{\star} o^{\top}\right)$ und teilweise auch denen von $P$. crassiorella und $P$. casta (PAllas, 1767) ( $\sigma^{\top} \sigma^{\star}$ und 우 우) (vergl. Taf. 9).

Für die molekular genetischen Untersuchungen lagen Imagines mit folgenden Daten vor:

$1 \sigma^{*}$, Greece, Kefalonia, Myrto Bay, e.l. 02.05.2005 (BC TMLF Lep 11169) (R. ionica nov. spec),

1 O$^{\star}$, Romania, Mţ. Câpâțenei, Chiaculata, e.l. 29.05.2011 (BC POESE 057) (R. arnscheidi),

1 ㅇ, Bulgaria, Umg. Sandanski, Liljanovo, e.1. 29.07.2015 ((BC POESE 059) (R. nigrociliella).

Im Ergebnis zeigt sich, dass die genetischen Differenzen beträchtlich sind. $R$. ionica spec. nov. unterscheidet sich um $6,55 \%$ von $R$. nigrociliella und um $7,59 \%$ von R. arnscheidi.

Biologie und Ökologie: An den Hängen des Skoloupas oberhalb der Myrto Bay (Fig. 13) flogen am 14.05.2002 auf ein frisch geschlüpftes 우 von Vliko (Lefkada) zwischen 09:25 und 10:10 Uhr (OESZ) insgesamt $6 \sigma^{\top} o^{\top}$ an. Einen Tag später waren auch die geschlüpften $\sigma^{\star} \sigma^{\top}$-Säcke i.M. dort zu finden. Am 16.05. war der Autor abermals vor Ort. Gleich bei der Ankunft um 09:05 Uhr begann wieder der Anflug auf zwei +9 von Vliko. Insgesamt flogen bis 09:40 Uhr $29 \sigma^{\star} o^{\star}$ an und eine Kopula wurde zugelassen. Die Weibchen stellten das Locken zu diesem Zeitpunkt ein. Wenig später fand sich ein im Schatten einer Felswand ein fliegendes $\sigma^{\star}$ um 10:08 Uhr (alles OESZ). Aus den Säcken der wohl befruchteten Weibchen konnte später kein Schlupf der F1 (Räupchen) festgestellt werden. Die Beobachtungen erfolgten bei strahlendem Sonnenschein und Temperaturen um 15 Grad Celsius.

Bei der Zucht fraßen die Raupen verschiedene Pflanzen, wie Hieracium spec. und Plantago spec. zumeist im angewelkten Zustand. Der Schlupf der $\sigma^{*} \sigma^{*}$ ist zwischen dem 06.05. und 12.06. beobachtet worden, der der 우 우 vom 08.05. bis sogar Anfang Juli. Beide Geschlechter schlüpften in den ersten Morgenstunden. Die Phänologie von $R$. ionica spec. nov. ist jahrweise relativ konstant, zieht sich aber über einen längeren Zeitraum hin. Die Eiablage erfolgt in den Sack und die Art ist univoltin.

Vergesellschaftet ist $R$. ionica spec. nov. quasi mit allen auf der Insel nachgewiesenen Psychidenarten, ausser mit B. cephalonica spec. nov. (vergl. Tab. 1).

Die Habitate bilden zumeist Felsabbrüche und -fluren, aber auch an Trockenmauern (Dolomit und Kalkstein), die xerothermen Charakter und Maccia-Vegetation aufweisen und häufig nach SE ausgerichtet sind. Zumeist liegen diese Standorte ab dem Nachmittag im Schatten.

\section{Reisseronia ionica odysseus subspec. nov.}

Derivatio nominis: Die neue Subspies wird nach Opysseus, dem Helden der griechischen Mythologie benannt, der nach der Ilias von Homer von dieser Insel stammte.

Holotypus: ${ }^{\star}$, Europa meridionalis, Greece-West/Ithaka, Ionische Inseln, Umg. Lefki $3 \mathrm{~km} \mathrm{S,} \mathrm{200-300} \mathrm{m} \mathrm{NN,} \mathrm{e.l.}$ 29.05.2003, leg. Dr. M. WeidLICH (Flügelspanne 8,9 mm) (Fig. 5 b). Er befindet sich in coll. Museum für Naturkunde des Leibniz Institutes in Berlin.

Paratypen (die gezüchteten Exemplare jeweils mit Sack und Puppenhülle): Männchen: $24 \sigma^{\top} \sigma^{\star}$, e.l. 15.05.03.06.2000; $11 \sigma^{\star} o^{\top}$, e.p. 06.05.-09.06.2001; $3 \sigma^{\top} o^{\top}$, e.l. 20.05.-03.06.2002; $4 \sigma^{\star} \sigma^{\star}$, e.l./e.p. 17.05.-06.06.2003; $16 \sigma^{\top} \sigma^{\top}$, e.l./e.p. 05.05.-Anf.06.2005; $19 \sigma^{\star} \sigma^{\star}$, e.p./e.l. 11.05.-07.06.2015: Europa meridionalis, Greece-West, Ithaka, Umg. Lefki $3 \mathrm{~km} \mathrm{~S} \mathrm{200-300} \mathrm{m} \mathrm{NN;} 6 \sigma^{\top} \sigma^{*}$, e.l. Ende 05.2001; $3 \sigma^{\top} \sigma^{\top}$, e.p. 15.-21.2002; $13 \sigma^{-1} \sigma^{-1}$, e.l. 26.05.-10.06.2003; $4 \sigma^{\star} \sigma^{\star}$, e.l 20.-30.05.2005: Europa meridionalis, Greece-West, Ithaka, Vathi-Zentrum,

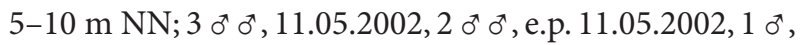
e.l. 14.05.2002; 1 क , e.p. 16.05.2003: Europa meridionalis, Greece-West, Ithaka, Perahori 1,5 km oberh., $350 \mathrm{~m} \mathrm{NN}$; $15 \sigma^{\top} \sigma^{\star}$, e.l. 11.-26.05.2000, $7 \sigma^{\star} \sigma^{\star}$, e.p. 09.-30.05.2001; $8 \sigma^{\star} \sigma^{\star}$, e.l. 28.05.-06.06.2003; $6 \sigma^{\star} \sigma^{\star}$, e.l. 03.-21.05.2005; $2 \sigma^{\top} \sigma^{\star}$, e.l. 21.05.2015: Europa meridionalis, Greece-West, Ithaka, Umg. Stavros S, 100-120 m NN;

$9 \sigma^{\top} \sigma^{\star}$, e.1. 22.-29.05.2000: Europa meridionalis, GreeceWest, Ithaka, Frikes, 5-20 m NN; $2 \sigma^{\star} \sigma^{\star}$, e.l. 30.05.2003, $1 \sigma^{\star}$, e.l. 31.05.2003: Europa meridionalis, GreeceWest, Ithaka, Kalamos-Quelle, $150 \mathrm{NN} ; 3 \sigma^{\star} o^{\star}$, e.p./ e.l. 18.-28.05.2001; $13 \sigma^{\top} \sigma^{\star}$, e.p./e.1. 12.05.-05.06.2002; $8 \sigma^{\star} \sigma^{*}$, e.p. 09.05.-01.06.2003; $2 \sigma^{*}$, e.l. 15.05.2005, $1 \sigma^{\star}$, e.l. 28.05.2005; $20 \sigma^{\top} \sigma^{\star}$, e.l. 20.05.-01.06.2015: Europa meridionalis, Greece-West, Ithaka, Exogi unterhalb, $300 \mathrm{~m} \mathrm{NN}$, alles leg. Dr. M. WEIDLICH.

Weibchen: 12 우 우, e.l. 20.-28.05.2000; 21 우 우, e.p. 09.05.-30.05.2001; 1 ㅇ, e.l. 28.05.2003; 7 ㅇ 우, e.l. Anf.05. -Anf.06.2005; 16 우 우, e.p./e.l. 21.05.-05.06.2015 (Fig. 6): Europa meridionalis, Greece-West, Ithaka, Umg. Lefki $3 \mathrm{~km} \mathrm{~S} \mathrm{200-300} \mathrm{m} \mathrm{NN;} 2$ 우, e.l. 21.05.2003, 1 ㅇ, e.l. 07.06.2003: Europa meridionalis, Greece-West, Ithaka, Perahori 1,5 km oberh., $350 \mathrm{~m} \mathrm{NN}$;

36 우 우, e.1. 15.05.-03.06.2000; 16 우 우, e.p. 11.-30.05.2001; 1 우 e.p. 10.05 .2002 , 1 ㅇ e.l. $01.06 .2002 ; 1$ 우 e.l. 22.05.2003, 1 우 e.l. 30.05 .2003 ; 1 우 e.l. 30.05 .2005 ; 1 우, 08.06.2015: Europa meridionalis, Greece-West, Ithaka, Umg. Stavros S, 100-120 m NN; 1 ㅇ, e.l. 02.06.2001, 1 ㅇ, e.l. 21.05.2003, 5 우 우, e.l. Anf.06.2005; 7 우우, e.p./e.l. 11.-23.05.2015: Europa meridionalis, GreeceWest, Ithaka, Exogi unterhalb, $300 \mathrm{~m} \mathrm{NN}$, alles leg. Dr. M. WeIDLICH. 
Säcke: 53 Säcke, 13.07.1993; 29 Säcke, 26.04.2000; 640 Säcke 03.-06.05.2001; 73 Säcke, 09.05.2002; 37 Säcke, 03.05.2003; 154 Säcke, 07.04.2005; 83 Säcke, 06.05.2015: Europa meridionalis, Greece-West, Ithaka, Umg. Lefki 3 km S 200-300 m NN; 6 Säcke, 03.05.2001; 29 Säcke, 13.05.2002; 18 Säcke 04.05.2003; 11 Säcke, 08.04.2005: Europa meridionalis, Greece-West, Ithaka, VathiZentrum, 5-10 m NN; 75 Säcke, 11.05.2002, 20 Säcke 04.05.2003; Europa meridionalis, Greece-West, Ithaka, Perahori 1,5 km oberh., $350 \mathrm{~m} \mathrm{NN}$;

60 Säcke, 28.04.2000; 8 Säcke, 03.-7.05.2001; 29 Säcke, 10.05.2002; 40 Säcke, 03.05.2003; 24 Säcke, 08.04.2005; 15 Säcke, 06.05.2015: Europa meridionalis, Greece-West, Ithaka, Umg. Stavros S, 100-120 m NN;

7 Säcke, 27.04.2000; 10 Säcke, 04.05.2001: Europa meridionalis, Greece-West, Ithaka, Frikes, 5-20 m NN; 2 Säcke, 08.04.2005: Europa meridionalis, Greece-West, Ithaka, Kioni 0,5 km NW, 30 m NN; 1 Sack 09.04.2005, 40 Säcke, 07.05.2015: Europa meridionalis, Greece-West, Ithaka, Piso Aetos, $5-20 \mathrm{~m} \mathrm{NN}$; 3 Säcke, 03.05.2003: Europa meridionalis, Greece-West, Ithaka, Kalamos-Quelle, 150 m NN; 34 Säcke, 07.05.2001; 49 Säcke, 12.05.2002; 40 Säcke, 03.05.2003; 29 Säcke 08.04.2005; 35 Säcke 06.05.2015: Europa meridionalis, Greece-West, Ithaka, Exogi unterhalb, $300 \mathrm{~m} \mathrm{NN}$, alles leg. Dr. M. WEIDLiCH.

Das Typenmaterial umfasst somit $208 \sigma^{\top} \sigma^{\star}$ und 132 우 웅 die gezüchteten Ex. jeweils mit Sack und Puppenhülle sowie 1654 Säcke. Es befindet sich in den Sammlungen W. R. ARnscheid (Bochum/Deutschland), Naturkundemuseum der Leibniz Gesellschaft (Berlin/Deutschland), Senckenberg Gesellschaft für Naturforschung (Müncheberg/Deutschland), Tiroler Landesmuseen-Betriebsgesellschaft m.b.H. (Innsbruck/ Österreich) und Z. ToKÁr (Michalovce/Slowakei).

Diagnose: Männchen: Mittelgroße Falter mit einer Flügelspanne von 8,9 bis $11,4 \mathrm{~mm}$, Färbung einfarbig schwarzbraun. Augen schwarz, rund, und der Augenindex (Quotient auf aus Augenabstand bis maximalen Augendurchmesser beträgt 1,0 bis 1,1. Nebenaugen (Ocellen) nicht vorhanden. Kopfbehaarung schwarzbraun, Labialpalpen kurz und rudimentär.

Die Fühlerlänge kurz, erreicht etwa die Hälfte der Länge des Vorderflügelcostalrandes. Fühlergliederzahl 21-22, Fühlerglieder dicht beschuppt, Kammzähne mit Wimpern versehen, deren Länge etwa der Kammzahnbreite entspricht. Die Kammzähne erreichen fast das Vierfache der Länge der Fühlerglieder (mittlerer Fühlerbereich).

Vorderflügel breit, Färbung einfarbig schwarzbraun, Diskoidalfleck nicht erkennbar. Deckschuppen schmal: ein- und zweizackig, selten dreizackige Schuppen (Klasse I-II nach SAUTER, 1956). Vorderflügel mit Eingeschobener Zelle, ohne Anhangszelle, 8 Diskoidalzelladern, die alle, getrennt voneinander der Diskoidalzelle entsprin- gen. Fransen einfarbig schwarzbraun, leicht kupfrig schimmernd, lancettförmig

Hinterflügelfärbung wie die der Vorderflügel, 5 Diskoidalzelladern, die alle getrennt voneinander entspringen. Hinterflügel mit haarförmigen, lancettförmigen, zweizackigen und selten dreizackigen Schuppen bedeckt. Fransen leicht kupfrig schimmernd, Fransenschuppen lancettförmig, aber schmaler als die der Vorderflügelfransen. Körper schwarzbraun behaart.

Vordertibie ohne Epiphyse, Mitteltibie und Hintertibie mit jeweils einem Spornpaar, alle Beine mit 5 Tarsengliedern. Genitalarmatur Reisseronia-typisch und von rundlicher Gestalt. Die Valven überragen das Tegumendach, welches zweifach leicht eingekerbt ist. Clavus deutlich ausgebildet mit Cornuti. Anelli relativ klein und schmal und spitz ausgebildet. Vinculum breit und rundlich gestaltet ohne Saccus. Phallus gerade, kräftig und erreicht fast die Valvenlänge, ohne Cornuti (Fig. 8 b, e).

Weibchen: Flügellos, gelblich bis hellbräunlich, tw. rötlichbraun, Körperlänge 6-7 mm in gestreckter, natürlicher Haltung (ohne Ovipositor), -durchmesser etwa $2 \mathrm{~mm}$. Augen klein schwarz und rund, keine Ocellen, Fühler rudimentär, 2 gliedrig, Tarsen dreigliedrig. Schüttere Körperbehaarung, Afterwollhaare silbrig und kranzförmig am 7. Segment vorhanden.

Sack: Säcke in den Abmaßen sehr unterschiedlich, ein Geschlechtsdimorphismus ist bei ihnen auch in den letzten Raupenstadien zu erkennen: Männchen: Länge 6-9 mm, Breite: 2,0-3,5 mm (Fig. 9 c); Weibchen: Länge 12-15, Breite 3-4 mm (Fig. 9 g) (jeweils adulte Säcke von geschlüpften Imagines ohne überstehendes Baumaterial). Der Sack ist sehr unregelmäßig aufgebaut und erinnert an den von Psyche- und Proutia-Arten. Er besteht wie bei $R$. ionica spec. nov. hauptsächlich aus Grashalmen, Blattresten und untergeordnet aus Blüten- und Samenteilchen. Auch kleine Mineralteilchen und Kalkplättchen wurden zum Bau verwendet. Ihre Färbung reicht von mittelgrau bis bräunlich.

Biologie und Ökologie: Im Freiland wurden geschlüpfte männliche Säcke ab dem 03.05. angetroffen (Stavros und Kalamos-Quelle). Bei Perahori flogen am 11.05.2002 bei leicht windigem, bedecktem Wetter (17 Grad Celsius) zwischen 13:35 und 13:40 Uhr $4 o^{\star} o^{\star}$ an einem Felshang. Einen Tag später konnte der Schlupf zweier $\sigma^{*} \sigma^{*}$ aus bei Exogi eingetragenem Zuchtmaterial zwischen 14:47 und 15:30 Uhr (beides OESZ) beobachtet werden. Bei der Zucht ist der Schlupf der o $^{\top} \sigma^{\top}$ zwischen dem 05.05. und 10.06., der der o 9 im Zeitraum vom 09.05. bis 08.06. nachgewiesen werden. Die Raupen ernähren sich ebenfalls wie die von $R$. ionica spec. nov. von niedrigen Pflanzen.

Auf Ithaka kommt R. ionica odysseus subspec. nov. verbreitet vor und besiedelt die Insel vom Meeresspiegelniveau bis auf $350 \mathrm{~m} \mathrm{NN}$. Lebensräume bilden wie bei $R$. ionica spec. nov. xerotherme Felsabbrüche und -fluren 
in der Maccia-Vergetation und auch stellenweise lebt die subspec. nov. in großen Populationen z.B. bei Lefki (Fig. 14). Bemerkenswert ist die häufig SW ausgerichtet Lage der Felsabbrüche, die die Hypothese stützen kann, dass odysseus subspec. nov. kein Frühflieger sondern ein Nachmittagflieger ist (vergl. oben).

\section{Reisseronia ionica lefkadensis subspec. nov.}

Derivatio nominis: Die neue Art wird nach der Insel Lefkada im Ionischen Meer benannt, auf der sie offenbar endemisch vorkommt.

Holotypus: $0^{\star}$, Europa meridionalis, Greece-West, Lefkada, Ionische Inseln, Katohori. $150 \mathrm{~m} \mathrm{NN}$. e.o. 15.05.2013, leg. Dr. M. Weidlich (Flügelspanne 8,2 mm) (Fig. 5 d). Er befindet sich in coll. Museum für Naturkunde des Leibniz Institutes in Berlin.

Paratypen (die gezüchteten Exemplare jeweils mit Sack und Puppenhülle): Männchen: $21 \sigma^{\top} \sigma^{\top}$, e.l./e.p. 10.05.16.06.2002; $5 \sigma^{\star} \sigma^{*}$, e.l./e.p. 17.05.-01.06.2003; $5 \sigma^{\star} \sigma^{*}$, e.l./e.p. 28.04.-19.05.2005: Europa meridionalis, GreeceWest, Lefkada,Vliko 0,5 km S, $70 \mathrm{~m} \mathrm{NN} ; 13 \sigma^{7} \sigma^{\star}$, e.l./e.p. 07.-31.05.2003; $22 \sigma^{*} \sigma^{*}$, e.p./e.1. 03.-17.05.2012; $41 \sigma^{*} \sigma^{*}$, e.o. 15.05.-09.06.2013 (Fig. 7); 7 o $^{\top}{ }^{\top}$, e.p. 10.-18.05.2015: Europa meridionalis, Greece-West, Lefkada,Umg. Katohori, 150 m NN, alles leg. Dr. M. WEIDLICH.

Weibchen: 42 우, e.1./e.p. 11.-30.05.2002; 8 우, e.1./ e.p. 09.-27.05.2002: Europa meridionalis, Greece-West, Lefkada,Vliko $\quad 0,5 \mathrm{~km} \mathrm{~S}, 70 \mathrm{~m} \mathrm{NN} ; 14$ ㅇ,$\quad$ e.l./e.p. 21.-14.06.2003; 26 우 우 e.o. 15.05.-05.06.2013; 1 우 e.p. 10.05 .2012 ; 1 ㅇ , e.p. $16.05 .2015,1$ 우 , e.p. 21.05.2015: Europa meridionalis, Greece-West, Lefkada,Umg. Katohori, 150 m NN, alles leg. Dr. M. WEIDLICH.

Säcke: 147 Säcke, 08.05.2002; 25 Säcke 05.04.2003; 17 Säcke, 09.04.2005; 24 Säcke, 21.04.2012: Europa meridionalis, Greece-West, Lefkada,Vliko 0,5 km S, 70 m NN; 57 Säcke, 08.05.2003; 22 Säcke, 09.04.2005; 14 Säcke, 21.04.2012; 46 Säcke, 22.04.2012; 69 Säcke, e.o. 06.2013; 16 Säcke, 03.-04.05.2015: Europa meridionalis, GreeceWest, Lefkada, Umg. Katohori, 150 m NN; 4 Säcke, 09.04.2005: Europa meridionalis, Greece-West, Lefkada, Umg. Elithea $1 \mathrm{~km} \mathrm{S,} 600 \mathrm{~m} \mathrm{NN}$; 1 Sack, 09.04.2005: Europa meridionalis, Greece-West, Lefkada, Umg. Kamilio S Kirche, $500 \mathrm{~m}$ NN, alles leg. Dr. M. WeIdLICH.

Das Typenmaterial umfasst somit $115 \sigma^{\star} \sigma^{\star}$ und 93 ㅇ 우, die gezüchteten Ex. jeweils mit Sack und Puppenhülle sowie 442 Säcke. Es befindet sich in den Sammlungen W. R. ArNscheId (Bochum/Deutschland), Naturkundemuseum der Leibniz Gesellschaft (Berlin/Deutschland), Senckenberg Gesellschaft für Naturforschung (Müncheberg/Deutschland), Tiroler
Landesmuseen-Betriebsgesellschaft m.b.H. (Innsbruck/ Österreich) und Z. TokÁr (Michalovce/Slowakei).

Diagnose: Männchen: Kleine Falter mit einer Flügelspanne von 8,1 bis $10,0 \mathrm{~mm}$, Färbung einfarbig schwarzbraun. Augen schwarz, rund und der Augenindex beträgt 1,0 bis 1,1 . Nebenaugen (Ocellen) nicht vorhanden. Kopfbehaarung schwarzbraun, Labialpalpen kurz und rudimentär.

Die Fühlerlänge relativ kurz, erreicht knapp die Hälfte der Länge des Vorderflügelcostalrandes. Fühlergliederzahl 20-22, Fühlerglieder dicht beschuppt, Kammzähne mit Wimpern versehen, deren Länge etwa der Kammzahnbreite entspricht. Die Kammzähne erreichen fast das Vierfache der Länge der Fühlerglieder (mittlerer Fühlerbereich).

Vorderflügel breit, Färbung einfarbig schwarzbraun, Diskoidalfleck nicht erkennbar. Deckschuppen schmal: ein- bis dreizackig, die dreizackigen Schuppen selten (Klasse I-II nach SAUTER, 1956). Vorderflügel mit Eingeschobener Zelle, ohne Anhangszelle, 8 Diskoidalzelladern, die alle getrennt voneinander der Diskoidalzelle entspringen. Fransen einfarbig schwarzbraun, leicht kupfrig schimmernd, lancettförmig, einzackig.

Hinterflügelfärbung wie die der Vorderflügel, 5 Diskoidalzelladern, die alle getrennt voneinander entspringen. Hinterflügel mit haarförmigen, lancettförmigen, zweizackigen und selten dreizackigen Schuppen bedeckt. Fransenschuppen lancettförmig, aber schmaler als die der Vorderflügelfransen. Körper schwarzbraun behaart. Vordertibie ohne Epiphyse, Mitteltibie und Hintertibie mit jeweils einem Spornpaar, alle Beine mit 5 Tarsengliedern. Genitalarmatur Reisseronia-typisch und von rundlicher Gestalt.

Valven überragen das Tegumendach, welches zweifach leicht eingekerbt ist. Clavus deutlich ausgebildet mit Cornuti. Anelli auffallend breit und stumpf, Vinculum breit und rundlich gestaltet ohne Saccus. Phallus gerade, kräftig und erreicht fast die Valvenlänge, ohne Cornuti (Fig. $8 \mathrm{c}, \mathrm{f}$ ).

Weibchen: Flügellos, gelblich bis hellbräunlich, tw. rötlichbraun, Körperlänge 6-7 mm in gestreckter, natürlicher Haltung (ohne Ovipositor), Körperdurchmesser etwa $2 \mathrm{~mm}$. Augen klein schwarz und rund, keine Ocellen, Fühler rudimentär, zweigliedrig, Tarsen dreigliedrig. Schüttere Körperbehaarung, Afterwollhaare silbrig und kranzförmig am 7. Segment vorhanden.

Sack: Säcke in den Abmaßen sehr unterschiedlich, ein Geschlechtsdimorphismus ist bei ihnen ebenfalls in den letzten Raupenstadien zu erkennen: Männchen: Länge 7-9 mm, Breite: 2,5-3,5 mm (Fig. 9 d); Weibchen: Länge 12-18 mm, Breite 3-4 mm (Fig. 9 h) (jeweils adulte Säcke von geschlüpften Imagines ohne überstehendes Baumaterial).

Der Sack ist sehr unregelmäßig aufgebaut und erinnert ebenfalls an die von Psyche- und Proutia-Arten. Er 
besteht, wie bei $R$. ionica spec. nov. und $R$. ionica odysseus subspec. nov., hauptsächlich aus Grashalmen, Blattresten und untergeordnet aus Blüten- und Samenteilchen. Die Färbung reicht von mittelgrau bis bräunlich.

Differentialdiagnose von $R$. ionica spec. nov., $R$. ionica odysseus subspec.nov. und $R$. ionica lefkadensis subspec. nov.: Die drei Taxa unterscheiden sich in der Größe der $o^{\top} o^{\star}$ voneinander. $R$. ionica spec. nov. ist deutlich größer als als odysseus subspec. nov., wenngleich es einen Überschneidungsbereich zwischen 10,6 und 11,4 mm Flügelspanne gibt. R. lefkadensis subspec. nov. ist die kleinste der drei genannten Taxa, welche aber auch mit einem Überschneidungsbereich mit odysseus subspec. nov. zwischen 8,9 bis $10,0 \mathrm{~mm}$ ausgestattet ist. Frisch geschlüpfte $o^{\star} o^{\star}$ erscheinen etwas dunkler schwarzbraun (Fig. 7) als die beiden anderen Taxa.

Im männlichen Genitalbereich lassen sich auch geringfügige Unterschiede feststellen. Vor allem in der Form der Anelli, die bei lefkadensis subspec. nov. deutlich breiter als bei den beiden anderen Taxa ausgebildet sind.

Genetische Untersuchungen wurden ebenfalls an den drei beschriebenen Reisseronia-Taxa in den Jahren 2013 und 2014 von CCDB Ontario, Canada durchgeführt. Als Untersuchungsmaterial lagen $50^{\star} o^{\star}$ mit folgenden Daten vor:

Kefalonia, Myrto Bay, e.l. 02.05.2005 (BC TMLF Lep 11169), e.l. 04.05.2005 (BC TMLF Lep 07220), Ithaka, Umg. Exogi, e.l.15.05.2005 (BC TMLF Lep 11168), Ithaka, Umg. Lefki, e.l. 19.05.2005 (BC TMLF Lep 07221) und Lefkada, Umg. Katohori, e.l. 16.05.2013 (BC TMLF Lep 11167).

Im Ergebnis unterscheiden sich sich die drei Taxa wie folgt:

Die beiden $\sigma^{\top} \sigma^{\star}$ der Population von Kefalonia unterscheiden sich um 2,4 \% von den beiden Taxa der Inseln Ithaka und Lefkada. Diese wiederum liegen genetisch nahe beieinander und weisen lediglich 0,8\% Unterschiede in der mtDNA auf. Aufgrund dieser Analyse in Kombination mit den morphologischen Merkmalen sowie dem Anflugverhalten kann geschlussfolgert werden, dass die drei Taxa einer Art angehören und die Populationen von Ithaka wie auch von Lefkada als subspezifisch gelten.

Interessant ist ausserdem, dass bezogen auf $R$. nigrociliella, die genetischen Differenzen der Populationen der von Ithaka geringer und die von Lekada nochmals geringer sind als die von Kefalonia.

Bisher ungeklärt ist, warum R.ionica odysseus subspec. nov. eine andere Biologie aufweist. Ihre $\sigma^{*} \sigma^{*}$ fliegen nachmittags und auch die $q \circ$ locken in diesem Zeitraum. R. ionica spec. nov. und R. ionica lefcadensis subspec. nov. sind dagegen Morgenflieger, d.h. die $\sigma^{\top} o^{\star}$ fliegen vormittags, wie auch die $9+9$ in dieser Zeit aktiv sind. Dieses wäre ein bisher selten beobachtetes Verhalten bei Psychiden ein und derselben Art. Vielleicht ist davon auszugehen, dass es sich um zwei Flugzeiten handelt, eine am Morgen/Vormittag und eine am Nachmittag. Eine derartige Lebensweise ist bei Loebelia crassicornis
(STAUdinger, 1871) nachgewiesen worden (WEIDLICH, 2012a: 84). Weitere Beobachtungen müssen hier Klarheit verschaffen.

Die 우 und die Säcke der drei Taxa lassen sich kaum voneinander differenzieren.

Biologie und Ökologie: Auf Lefkada wurden die ersten geschlüpften männlichen Säcke im Freiland am 05.05. gefunden (Katohori). Insgesamt gesehen, konnte bei der Zucht der Schlupf der $\sigma^{\top} \sigma^{\star}$ zwischen dem 05.05. und 10.06., der 우 우 im Zeitraum vom 09.05. bis zum 08.06. nachgewiesen werden. Die Raupen ernähren sich ebenfalls wie die von $R$. ionica spec. nov. und $R$. ionica odysseus subspec. nov. von niedrigen Pflanzen.

Eine Eizucht aus Katohori verlief relativ problemlos. Die Eiräupchen schlüpften ab dem 01.06.2012. Den Winter überstanden die Raupen in einem Terrarium mit wenig Verlusten. Der Schlupf begann am 15.05. und zog sich bis zum 09.06.2013 hin. Gefüttert wurden die Raupen, mit Achillea millefolium, Plantago lanceolata, Trifolium spec. sowie Gräsern.

Auf Lefkada wurde R. ionica lefkadensis subspec. nov. nur an 4 Lokalitäten angetroffen. Ausschlaggebend für die eingeschränkte Verbreitung auf dieser Insel ist wahrscheinlich, dass nur an wenigen Stellen festes Kalkgestein ansteht, welches bevorzugt wird. Weit verbreitet sind hier weichere Sedimente. Die Habitate bilden ebenfalls Maccia-Bereiche in hier wiederum SE-ausgerichteten Felsabbrüchen (Fig. 15). Die Unterart wurde zwischen 70 und $600 \mathrm{~m} \mathrm{NN}$ beobachtet. Auf dem gegenüber liegendem Festland und auch im nahen Akarnanika-Gebirge wurde sie bisher nicht gefunden. Erwähnenswert ist, dass in Nachbarschaft an feuchteren Standorten nahe dem Festland und in einem Olivenhain Psyche crassiorella (BRUAND, 1850) vorkommt, die Ithaka und Kefalonia offenbar fehlen (siehe oben).

\section{Heliopsychidea graecella (MıLıIèRE, 1866)}

\section{Neu für die Ionischen Inseln.}

Vom Autor auf dem Enos-Gipfel gefunden: am 30.04. und 01.05.2001 6 Säcke: Schlupf eines + am 09.05.2001. Auch in den anderen Jahren (2002, 2005 und 2015) immer wieder vereinzelt dort Säcke gefunden. Ausserdem flogen am 08.05.2005 bei Stavros 5 Ex. zwischen 11:56 und 12:15 Uhr (OESZ) im Sonnenschein. Die nächsten Vorkommen der in Griechenland weit verbreiteten aber nicht oft beobachteten Psychide befinden sich im Akarnanika-Gebirge gegenüber Lefkada auf dem Festland (WEIDLICH, 2005 unveröffentlicht).

\section{Canephora hirsuta (PoDA, 1761)}

Neu für die Ionischen Inseln.

Nur von 4 Fundorten auf Ithaka und Lefkada, ab 2001, bekannt: Exogi, Frikes, Lefki und Vliko. 


\section{Pachythelia villosella (OCHSENHEIMER, 1810)}

Neu für die Ionischen Inseln.

Relativ verbreitet auf allen drei untersuchten Inseln und die Säcke meistens i.A. an folgenden Fundorten ab 1993: Anogi, Platrithias, Frikes, Exogi, Lefki, Vathi, Agios Efimia, Myrto Bay, Livadi, Vliko und Katohori. Diverse $0^{\pi} \sigma^{\pi}$ schlüpften zwischen den 07. und 21.05.

\section{Oiketicoides lutea (STAUDINGER, 1871)}

Rebel (1932:54) verweist auf einen Einzelfund von BEIER aus dem Jahre 1929 (als „Amicta febretta Boyer") von Krane (Kefalonia). Nach aktuellem Kenntnisstand kommt in Griechenland nur O. lutea vor. Dazu liegen folgende neuere Nachweise vor: Frikes: 7 Raupen am 27.04.2000, ein Sack am 04.05.2001, Stavros: ein Sack am 07.05.2001 und Lefki ein Sack am 06.05.2015.

\section{Apterona helicoidella (VALLot, 1827)}

Neu für die Ionischen Inseln.

Auf den drei vom Autor untersuchten Inseln weit verbreitet, so dass sich eine Aufzählung der Fundorte erübrigt; kommt in der parthenogenetischen Form vor.

\section{$\underline{\text { Incertae sedis }}$}

\section{„Epichnopteryx pulla Esp.“}

Rebel (1910: 426) vermerkt für dieses Taxon: „Ein ơ von Kephalonia (Argostoli, anfangs Mai, Hilf) läßt keinen Dorn an der Vorderschiene erkennen, gehört also wohl zu dieser Art".

\section{„? Psychidea bombycella Schiff."}

Nach Rebel $(1932: 54,55)$ ein einzelner Sackfund, den er als „wahrscheinlich hierher" gehörend einschätzt. Gesicherte Nachweise aus Griechenland liegen von dieser Art, d.h. Bijugis bombycella nicht vor und die nächsten, belegten Funde stammen aus Albanien (WeidLICH, 2013: 330). Jedoch können die Säcke von Heliopsychidea graecella denen von Bijugis bombycella (DeNis \& SCHIFFERMüLLER, 1775) durchaus ähneln.

\section{Yoponomeutidae}

\section{Argyresthia hilfiella REBEL, 1910}

Die Art wurde von Rebel nach einem $q$ von Charakti auf Kefalonia beschrieben. Offenbar gibt es keine neueren Funde und die Art muss nach bisherigen Erkenntnissen als endemisch gelten.

\section{Depressariidae}

\section{Agonopterix leucadensis (REBEL, 1932)}

Nach einem o aus Nidri (Lefkada) wurde die Art von Rebel (1932: 55) beschrieben. Auch von dieser wohl endemischen Art sind keine neuen Funde bekannt geworden.

\section{Coleophoridae}

\section{Coleophora niveicostella ZELLER, 1839}

Neu für die Ionischen Inseln.

Säcke in Anzahl bei Katohori und Vliko am 09.04.2005, aus denen am selben Tag 2 Ex. schlüpften.

\section{Zygaenidae}

Da es auch hier keine Gesamtübersicht über die griechischen Funde gibt, wird die Literatur ausgewertet und in Tab. 2 dargestellt. Finden sich bei De Freina \& WitT (2001) direkte Hinweise für die Ionischen Inseln, dann wird dieses Bestimmungswerk auch angeführt.

\section{Zygaena ephialtes (LINNAEUS, 1767)}

Von Erber auf Korfu gefunden (Staudinger, 1871: 105), auch in der gelben var. coronillae EsPER. Später von dort auch von Rebel (1912: (14)) und von Hynd (1981: 283), sie nennen jedoch nur die rote Form, nachgewiesen. Hofmann (2003) beschäftigt sich eingehend mit dem Z. ephialtis-Komplex im Mittelmeerraum. Ihm lag Material u.a. von Korfu, Lefkada, Ithaka und Kefalonia vor. Im Ergebnis der Untersuchungen wurde die subspec. odysseus Hofmann, 2003 beschrieben, die sich durch Individuen mit gelber Färbung und überwiegend 5 fleckigen Vorderflügeln auszeichnet (HoFmanN, 2003: 75, 109). Dieses Taxon kommt nur auf Kefalonia vor und fliegt dort von Anfang Juni bis Anfang Juli (Hofmann, 2003: 76). Jetzt liegt ein Einzelnachweis mit früherer Flugzeit vom 14.05.2002 bei Magganos (Kefalonia) vor.

Auf den benachbarten Inseln Lefkada und Ithaka kommen nur ausschließlich monochrom-rote Ex. vor. Dagegen gibt es auf Korfu rot-gelb gemischte Formen (vergl. Hofmann, 2003: 75). De Freina \& Piatkowski (2006: 258) führen die subspec. tymphestica HoLIK, 1948 für Lefkada (Sivota und Kalamitsi) an.

\section{Zygaena filipendulae (LINNAEUS, 1758)}

Von Korfu durch De la Garde $(1899: 9,11)$ und Rebel (1910: 426; 1912: (12)) genannt sowie in der subspec. 
(var.) ochsenheimeri ZELLER, 1847 von Lefkada mitgeteilt (Rebel, 1933: 91). Nach De Freina \& Witt (2001: 282) ist diese Unterart als Synonym von subspec. maior (ESPER, 1794) nur in Südfrankreich beheimatet. Ausserdem von Kefalonia (Argostoli) durch Rebel (1910: 426) und Vasillikadas, Defaranata, Skopoulas, Livadi (WeIDLICH) sowie von Zakynthos durch EMBACHER (2000: 66) bekannt. Neue Funde ab 2001 liegen auch von Ithaka vor, wo die Art stellenweise häufig vorkommt: Stavros, Vathi, Frikes, Marathias vom Monastir Taxiarchon und Lefki (WeIDLICH). Phänologisch wurde die Art, zwischen dem 03. und 14.05. (Ithaka und Kefalonia) und 28.05. (Zakynthos) beobachtet. Die Art kommt auf den Ionischen Inseln teilweise in aussergewöhnlich großen Ex. (bis $39 \mathrm{~mm}$ ) vor, welches bereits von REBEL (1912: (14)) bemerkt wird.

\section{Sesiidae}

Das Vorkommen etlicher Arten auf den Ionischen Inseln konnte bei DE FreinA (1999) nicht genau recherchiert werden, insbesondere bei den Angaben zu den beiden benachbarten Inseln Kefalonia und Zakynthos. Deshalb wurden diese Arten in Tab. 1 mit einem Fragezeichen versehen, aber trotzdem zum Faunenbestand gezählt. Weiterhin sind die Vorkommen von Tinthia myrmosaeformis (HERRICH-SCHÄFFER, 1846), Synansphecia muscaeformis (EsPER, 1783) und Chamaesphecia alysoniformis (HERRICH-SCHÄFFER, 1846) derzeit noch fraglich (LAŠTŮVKA in litt, 2016).

\section{Synansphecia umbrifera (STAUDINGER, 1871)}

Die von Korfu beschriebene Art (StAudinger, 1871: 96) und auch von ReBel (1924: 42) von dieser Insel genannt, zeichnet sich durch eine halobionte Lebensweise aus. Sie kommt nur hier und an den Küsten Mittelgriechenlands sowie auf der Peloponnes vor (DE FreINA 1999: 186, Tafel 14, 399, 418).

\section{Tortricidae}

\section{Obraztsoviana maculosana (HAWORTH, 1811)}

Neu für die Ionischen Inseln.

Durch LF. ein Einzelstück am 06.05.2005 an der Ormos Kakogilos festgestellt (LF.) (Gen.det. BLACKSTEIN).

\section{Aethes spec.}

Von der Gattung Aethes Billberg, 1820 waren bisher von den Ionischen Inseln 2 Arten bekannt (STAUDINGER, 1871:218): tesserana (DENIS \& SCHIFFERMÜLLER, 1775) und francillana (FABRICIUs, 1794). Jetzt erschien ein am 05.05.2003 bei Vathi am Licht. Eine Genitaluntersuchung von Tokár erbrachte Ähnlichkeiten zu A. rubiginana (WALSINGHAM, 1903), die aber bisher nur auf Sizilien, Italien und Gibraltar gefunden wurde (www.lepiforum.de). Deshalb wird das Taxon nicht in den Faunenbestand aufgenommen.

\section{Tortricodes alternella (DENIS \& SCHIFFERMÜLLER, 1775)}

\section{Neu für Griechenland.}

2 Ex. erschienen am 05.04.2005 bei Monastiráki (LF.) und ein Ex. nahe der Ormos Kakogilos (LF.) (Gen.det. BLACKSTEIN).

\section{Cnephasia cupressivorana (STAUDINGER, 1871)}

Neu für die Ionischen Inseln.

Von Staudinger (1871: 215) als eine „v.?“, also Varietät von wahlbomiana (= Pseudosciaphila branderiana (Linnaeus, 1758) beschriebenes Taxon aus Griechenland. C. cupressivorana ist nur genitaliter sicher zu bestimmen. Nunmehr auch von den Ionischen Inseln nachgewiesen, 5 Ex. erschienen beim Monastery Theotokou (04.04.2005) und bei Monastiráki (05.04.2005) am Licht (Gen.det. BLACKsTEIN).

\section{Lobesia botrana (DENIS \& SCHIFFERMüLLER, 1775)}

Neu für die Ionischen Inseln.

Ein Einzelstück erhielt der Verfasser am 05.05.2003 bei Vathi am Licht.

\section{Epinotia thapsiana (ZELLER, 1847)}

Nach Rogenhofer von Erber auf Korfu gefunden (STAUDINGER, 1871: 222). Jetzt ein ebenfalls Einzelstück am 05.05.2003 bei Vathi am Licht.

\section{Blastesthia tessulatana (STAUDINGER, 1871)}

Neu für die Ionischen Inseln.

Von Vathi durch LF. am 05.05.2003 und von Monastiráki am 05.04.2005 in je 1 Ex. nachgewiesen (LF.).

\section{Phalonidia albipalpana (ZeLLER, 1847)}

Neu für die Ionischen Inseln.

Unter www.lepiforum.de wird ein Nachweis für Lefkada erbracht (vergl. Foto vom 24.07.2014, leg. GRAF). 


\section{Prochoreutis stellaris (ZelLeR, 1847)}

Neu für die Ionischen Inseln.

M. Friedrich fand die Art auf Korfu bei Dassia (29.08.2011) (vergl. Foto unter www.lepiforum.de).

\section{Pterophoridae}

Oxyptilus distans (ZelLeR, 1847)

Neu für die Ionischen Inseln.

Es liegt ein Einzelstück von Vathi vor: LF. 25./26.04.2000.

Stangeia siceliota (ZELLER, 1847)

Neu für die Ionischen Inseln.

Ebenfalls ein Einzelstück von Vathi: LF. 04.05.2001.

\section{Pterophorus pentadactyla (LINNAEUS, 1758)}

Neu für die Ionischen Inseln.

Am Tage ein Ex. an einer Felswand ruhend: 14.05.2002 Myrto Bay.

\section{Pyralidae}

Hypotia infulalis LEDERER, 1858

\section{Neu für Griechenland.}

KARSHOLt \& Razowski (1996) geben die Art noch nicht für Griechenland an. Jetzt von GRAF am 24.07.2014 bei Lefkada gefunden (vergl. Foto unter www.lepiforum.de).

\section{Oncocera semirubella (SCOPOLI, 1763)}

Neu für die Ionischen Inseln.

Ein Einzelstück fand DAHL am 24.09.2007 am Robella Beach am Licht (Roda, Korfu, in litt. 2016).

Anerastia lotella (HÜBNER, 1813) wird bei STAUDINGER (1871:211) für Korfu angegeben. KARsholT \& Razowsky (1996) stellen Nachweise aus Griechenland als fraglich dar, weshalb die Art auch nicht in die Faunenliste aufgenommen wird.

\section{Crambidae}

Eucromius superbellus (ZelLeR, 1849)

Neu für die Ionischen Inseln.

Bei Kalamaki (Zakynthos) am 01.06.2012 nachgewiesen (EMBACHER in litt. 2016).

\section{Hydriris ornatalis (DUPONCHEL, 1832)}

Neu für die Ionischen Inseln.

Auf Korfu, Robolla Beach von DAHL gefunden (vergl. Foto unter www.lepiforum.de).

\section{Mecyna asinalis (HüBNER, 1819)}

Neu für die Ionischen Inseln.

Im Internet unter www.lepiforum.de fand sich der Erstnachweis für die Inselgruppe: Korfu, Ostküste, 01.-07.06.2008, leg. KRUmm.

\section{Palpita vitrealis (RossI, 1794)}

Neu für die Ionischen Inseln.

DAHL fand 2 Ex. am 24. und 26.09.2007 am Robolla Beach am Licht (Roda, Korfu) (in litt. 2016).

\section{Metasia ophialis (TREITSCHKE, 1829)}

Neu für die Ionischen Inseln.

Ein + flog am 06.05.2015 am Ormos Kaminia ans Licht.

\section{Lasiocampidae}

Malacosoma neustria (LINNAEUS, 1758)

Neu für die Ionischen Inseln.

Bei Vathi erschienen die Männchen i.A. (11.-12.05.2002) und am Ormos Kakogilos i.M. (15.-16.05.2002) am Licht. Die Raupen Ende April bis Mitte Mai sehr verbreitet und stellenweise häufig auf Kefalonia, Ithaka und Lefkada. Auch von Lefkada (Evgiris) aus dem Jahre 2003 gemeldet (De Freina \& Piatkowski, 2006: 250).

\section{Lemoniidae}

\section{Lemonia taraxaci (DenIs \& SCHIFFERMülLER, 1775)}

Neu für die Ionischen Inseln.

Entlang der Adriaküste südlich bis Griechenland kommt die subspec. strigata ReBeL, 1910 vor. Am 26.04.2000 eine Raupe bei Stavros eingetragen; Schlupf eines 우 am 12.10.2000.

\section{Sphingidae}

Marumba quercus (DeNIS \& SCHIFFERMüLLER, 1775)

Neu für die Ionischen Inseln. 
Zwei Einzelstücke bei Vathi (11.07.1993) und Ormos Kakogilos (15.05.2002) am Licht. Später im Jahr 2003 auf Lefkada (Evgiros) nachgewiesen (DE Freina \& Piatkowski, 2006: 252) und jetzt auch auf Zakynthos (Foto unter www.lepiforum.de, leg. PAPÉ).

\section{Hyles euphorbiae (LINNAEUS, 1758)}

STAudinger (1871: 89) führt Funde von Erber auf: „auf den von ihm besuchten Inseln“, so auch sehr wahrscheinlich von Korfu. Mittels LF. am 01.05.2003 bei Livadi nachgewiesen (1 Ex.).

\section{Hesperiidae}

Bei der Darstellung der Tagfalterarten aller dieser Familien in Tab. 1 wird weitgehend auf PAMPERIs (2009) verwiesen und es werden ältere Literaturquellen nicht gesondert aufgeführt. Jüngere Nachweise werden ergänzt und auch die Funde des Autors werden dargestellt.

\section{Muschampia proto (OCHSENHEIMER, 1808)}

In der Literatur für Vido bei Korfu (MATHEw, 1898: 112), für Korfu von BALDOCK \& BRETHERTON (1981:9), Embacher (2000: 67), PAMperis (2009: 624), PARker (2010: 41), DAHL (in litt. 2016), für Kythira von ReBEL (1937: 95) und PAMPERIs (2009: 624) genannt.

Die Raupen wurden teilweise in großer Zahl an Phlomis fruticosa am 12.05.2002 und 03.05.2003 bei Exogi, am 14.05.2002 bei Vasillikadas, am 04.05.2003 am Monastery Taxiarchon und Marathias sowie am 05.05.2015 bei Argostoli gefunden. Die Raupen spinnen die Blätter an den Spitzentrieben zusammen, fressen und verpuppen sich dort auch später. Die Falter schlüpften Anfang bis Ende August.

\section{Gegenes pumilio (HoffMANNSEgG, 1804)}

Zuerst von Staudinger (1871:88) genannt. Auch PAmperis (2009: 664) und PARker (2010: 41) geben mehrere Funde von Korfu an. EMBACHer (2000: 67) nennt die Art von Zakynthos (bei PAMPERIs nicht verzeichnet). Neue Funde gelangen auf Ithaka: 1 Ex. am 03.05.2001 an der Kalamos-Quelle und 2 Ex. am 04.05.2001 bei Frikes.

\section{Gegenes nostrodamus (FABRICIUS, 1793)}

Wird von MATHEW (1898: 112) von der kleinen Insel Vido bei Korfu angegeben. BALdock \& BRETHERTON (1981: 9) und PAMPERIs (2009: 667) stufen diese Funde als fraglich ein, zumal die häufigere und verbreitete G. pumilio von
Mathew nicht genannt wird. Deshalb wird die Art nicht zum Faunenbestand gerechnet.

\section{Pieridae}

\section{Colias myrmidone (ESPER, 1780)}

Norris (1891: 179) führt die Art für Korfu an, später von PAMPERIs (2009) aber nicht mehr genannt. Die Art wird deshalb nicht zur Fauna gerechnet.

\section{Colias caucasica StaUdinger, 1871}

PAMPERIS (2009: 112,113) verweist auf fragliche Mitteilungen von Korfu. Obwohl die Art in Nordwestgriechenland vorkommt, wird sie auch nicht in der Faunenliste aufgeführt.

\section{Colias alfacariensis RıвBE, 1905}

Von PAmperis (2009: 114) von Korfu verzeichnet und fraglich von Kefalonia/Zakynthos. Nun ein erster Fund auf Ithaka: am 04.05.2015 an den Westhängen des Skoloupas.

\section{Lycaenidae}

\section{Cupido argiades (PALLAS, 1771)}

Von Korfu als fraglich und nicht bestätigt gemeldet (PAMPERIS: 2009: 201), somit nicht zu Fauna gezählt.

\section{Scolitantides orion (PALLAS, 1771)}

Bereits von Staudinger (1871: 50) von Korfu genannt, von PAmperis (2009: 230) noch mit einem Fragezeichen für Korfu versehen. Jetzt 1 Ex. am 06.05.2015 bei Lefki auf Ithaka gefunden.

\section{Nymphalidae}

\section{Issoria lathonia (LINNAEUS, 1758)}

Offenbar nur wenig auf den Ionischen Inseln beobachtet. Papapavlou \& Katsouni (2008: 28) nennen ein Ex. vom Enos, PAMPeris (2009: 410) gibt Korfu und Kephalonia an, Zakynthos als fraglich. Der Autor kann ebenfalls eine Einzelbeobachtung von Enos mitteilen: 30.04.2001. 


\section{Brintesia circe (FABRICIUS, 1775)}

Je ein Einzelfund am 17.07.1993 und am 12.05.2002 bei Vathi. Sonst nur noch von Korfu erwähnt (PAMPERIS, 2009: 516 und DAHL in litt. 2016).

\section{Drepanidae}

\section{Habrosyne pyritoides (HuFNAGEL, 1766)}

Neu für die Ionischen Inseln.

Am 04.10.2007 fand DAHL ein Einzelstück am Robolla Beach (Roda, Korfu in litt. 2016).

\section{Polyploca ridens (FABRICIUS, 1787)}

Neu für die Ionischen Inseln.

Ein Einzelfund am 26.04.2000 bei Vathi am Licht.

\section{Watsonalla uncinula (BORKHAUSEN, 1790)}

Neu für die Ionischen Inseln.

Mehrfach auf den drei Inseln, teilweise i. A. im Zeitraum vom 30.04. bis 14.05. festgestellt: Vathi (erster Fund am 02.05.2001), Ormos Karini, Monastery Taxiarchon, Monastiráki, Aviathos lake, Nationalpark „Enos“ und Katohori/Vliko. Auch von PAPÉ auf Zakynthos festgestellt (Foto unter www.lepiforum.de).

\section{Geometridae}

\section{Godella aestimaria (HÜBNER, 1809)}

STAUdinger (1871: 159) meldet die Art von Korfu und neuerdings fand ЕмBACHER sie ebenfalls auf der Insel: Moraitika zwischen dem 11. und 17.07.1986 die Art (in litt. 2016).

\section{Neognopharmia stevenaria (BoISDUVAL, 1840)}

Neu für die Ionischen Inseln.

Zuerst 2001 auf Ithaka (Vathi) gefunden, dort mehrfach und teilweise i.A. zwischen dem 03.05. und 12.05. am Licht.

\section{Rhoptria asperaria (HÜBNER, 1817)}

Neu für die Ionischen Inseln.

Ebenfalls ab 2001 beobachtet. Mehrfach und stellenweise häufig am Licht, einzeln auch am Tage: Vathi, Exogi, Monastery Taxiarchon, Agios Eleftherios und bei Livadi (24.04.-14.05. mit einem Häufigkeitsmaximun Anfang Mai, so über 200 Ex. am 04.05.2003 bei Vathi).

\section{Pachycnemia hippocastanaria (HüBNER, 1799)}

Neu für die Ionischen Inseln.

Es erschienen bei Assos am 01.05.2001 über 50 Ex. am Licht. Ein Jahr später am 13.05.2002 dort nur ein Ex. Ein Einzelstück am 06.05.2015 am Ormos Kaminia (LF.).

\section{Nychiodes dalmatica (WAGNER, 1909)}

Neu für die Ionischen Inseln

Eine selten beobachtete Art. Je $1 \sigma^{\text {t }}$ bei Vathi am Licht: 03.05.2001 und 12.05.2002.

\section{Menophra abruptaria (Thunberg \& SeBALDT, 1792)}

Neu für die Ionischen Inseln.

Jeweils einzeln, selten i.A. ab 2001 durch Lichtfang festgestellt: Agios Eleftherios, Assos, Aviathos lake, Vathi, Ormos Kakogilos, Ormos Kaminia und Katohori (30.04.-12.05.).

\section{Menophra berenicidaria (TURATI, 1924)}

Neu für die Ionischen Inseln.

EMBACHER fand die Art zuerst auf Zakynthos bei Argassi (25.-27.05.1998, in litt. 2016). Ein Einzelstück ( $\overbrace{}^{\top}$ ) befand sich am 11.05.2002 in der Lichtfalle am Monastery Taxiarchon.

\section{Peribatodes umbraria (HüBNER, 1809)}

Neu für die Ionischen Inseln.

Relativ häufig zwischen dem 03.05. und 15.05. bei Vathi, Exogi, Monastery Taxiarchon, Ormos Kaminia, Vliko (bis zu 100 Ex. am 08.05.2002), Assos, Agios Eleftherios, Ormos Kakogilos nachgewiesen (LF., FL.). Neuerdings auch auf Korfu gefunden (vergl. Foto, leg. VonTz unter www.lepiforum.de).

\section{Peribatodes correptaria (ZeLleR, 1847)}

Neu für die Ionischen Inseln.

Relativ häufig zwischen dem 07.04. und 12.05. bei Vathi, Exogi, Monastery Taxiarchon, Vliko, Katohori, Assos und am Aviathos lake am Licht ab 2001 nachgewiesen. Auch auf Korfu gefunden (Fotos leg. DAHL und STRUTZBERG unter www.lepiforum.de).

\section{Ascotis selenaria (DenIs \& SCHIFFERMÜLLER, 1775)}

\section{Neu für die Ionischen Inseln.}

Wenige Einzelfunde ab 2001 gelangen am Licht zwischen dem 25.04. und dem 04.05. bei Vathi und am Aviathos lake. 


\section{Ectropis crepuscularia (DenIs \& SCHIFFERMüLLER, 1775)}

Neu für die Ionischen Inseln.

Ein Einzelnachweis auf Korfu: Robolla Beach 25.09.2007 (leg. DAHL in litt. 2016).

\section{Ematurga atomaria (LINNAEUS, 1758)}

Neu für die Ionischen Inseln.

Ebenfalls ein Einzelnachweis auf Korfu mit gleichen Daten wie bei E. crepuscularia (leg. DAHL in litt. 2016).

\section{Campaea honoraria (DeNIS \& SCHIFFERMüLlER, 1775)}

Neu für die Ionischen Inseln.

Zuerst auf Ithaka (Vathi) $1 \sigma^{\star}$ am 25./26.04.2000, 1 o 09.05.2002 und $1 o^{*}$ am 03.05.2003 durch LF. ermittelt Weiterhin $1 \sigma^{*}$ am Aviathos lake am 30.04.2003 und ein Pärchen am 04.05.2015 (LF).

\section{Pungeleria capreolaria (DeNIS \& SCHIFFERMüLleR, 1775)}

Die Falter sind i.A. aus dem Apollo-Tannenwald des Enos nachgewiesen worden, leg. Hilf (REBEL, 1910: 425). Fehlt bei Skou \& Sinvonen (2015: 397); Rebel (1910) wird auch nicht zitiert.

\section{Kemtrognophos onustaria (HERRICH-SCHÄFFER, 1852)}

Neu für die Ionischen Inseln.

Lediglich zwei Einzelstücke auf Lefkada und Ithaka: 08.05.2002 Vliko und 09.05.2002 Vathi gesammelt.

\section{Pseudoterpna pruinata (HufNAGEL, 1767)}

Zuerst von Erber auf Korfu gefunden (STAUdinger, 1871: 141). Ab 2001 mehrfach am Licht wie auch am Tage zwischen dem 03.05. und 12.05. bei Vathi gefunden.

\section{Euchristis indigenata (VILLERS, 1789)}

Neu für die Ionischen Inseln.

Ein + erschien am 08.05.2002 bei Vliko am Licht. Neuerdings auch von GrAF am 24.07.2014 bei Lefkada beobachtet (vergl. Foto unter www.lepiforum.de).

\section{Cosymbia suppunctaria (ZeLLER, 1847)}

Neu für die Ionischen Inseln.

Wiederum ein Einzelfund: 13.05.2002 bei Assos (LF.)

\section{Scopula submutata (TREITSCHKE, 1828)}

Neu für die Ionischen Inseln.

Mehrfach nachgewiesen, so bei Vathi, Exogi, Assos, Aviathos lake und Ormos Kakogilos ab 2000 jeweils am Licht sowie 1 Ex. tagsüber an Felsen bei Poros am 16.05.2002. Beobachtete Flugzeit: 25.04. bis 16.05.

\section{Scopula vigilata (WAGNER, 1926)}

Neu für die Ionischen Inseln.

Mehrfach bei Vathi am Licht zwischen dem 25.04. und 12.05. ab 2000 .

\section{Scopula rubiginata (HufNAGEL, 1767)}

Neu für die Ionischen Inseln.

Von DAHL am 24.09.2007 bei Roda, Robolla Beach gefunden (vergl. Foto unter www.lepiforum.de).

\section{Scopula marginepunctata (GoEZE, 1781)}

Neu für die Ionischen Inseln.

Verbreitet auf Kefalonia, so Myrto Bay (am Tage), am Ormos Kakogilos, am Aviathos lake, Livadi, Assos und auf Ithaka nur bei Vathi. Ab 2001 zwischen dem 30.04. und 15.05., zumeist am Licht.

\section{Scopula imitaria (HÜBNER, 1799)}

Staudinger (1871: 154), De la Garde (1899: 11) und Rebel (1910: 424) erwähnen die ersten Nachweise von Korfu, Kefalonia und Zakynthos; bei Hausmann (2004: 315) nicht aufgeführt. Immer nur einzeln am Licht, so bei Exogi, Assos, am Aviathos lake; bei Vathi etwas häufiger zwischen dem 06.04. und 13.05. Auch von DAHL (in litt. 2016) und von KRUMm (vergl. Foto unter www.lepiforum.de) auf Korfu gefunden.

\section{Scopula minorata (BoISDUVAL, 1833)}

Von Embacher (2000: 68) und von HausmanN (2004: 332) von Korfu aufgeführt. Die circummediterran verbreitete Art konnte auch mit zwei Einzelstücken von Ithaka (Vathi) belegt werden: 25./26.04.2000 und 02.05.2001 LF. Die beiden Ex. sind etwas größer und heller als die aus einer Serie vom 03.06.1983, leg. M. WEIDLICH aus Melnik bei Sandanski (Bulgarien).

Später auch auf Zakynthos (Vassilikos) von EMBACHER (in litt. 2016) gefunden (29.-30.05.2005). 


\section{Glossotrophia confinaria (HERRICH-SCHÄFFER, 1847)}

Bereits von STAUdinger (1871: 152) von Korfu genannt, fehlt bei Hausmann (2004: 345). Ein Ex. tags an den Felsen der Westhänge des Skoloupas und ein weiteres Ex. am Licht bei Assos (13.05.2002).

\section{Idaea filicata (HÜBNER, 1799)}

Auf den Ionischen Inseln weit verbreitet und häufiger als I. rusticata (DENIS \& SCHIFFERMüLLER, 1775). Bereits Rebel (1910: 424) verzeichnet die Art von Korfu, Zakynthos (von Hausmann, 2004: 87, 91 nicht aufgeführt) und Kefalonia. In mehreren Generation u.a. vom 05.04.-01.05. und 11.07.-16.07. verbreitet auf Lefkada, Ithaka und Kefalonia gefunden, meistens LF. (WEIDLICH). Auch von EMBACHER für Argassi (Zakynthos) mitgeteilt (in litt. 2016).

Idaea elongaria (RAMBUR, 1833)

Neu für die Ionischen Inseln.

EMbacher gibt zwei Funde von Zakynthos bekannt: Argassi (22.-27.05.1998) und Vassilikos (29.-30.05.2004) (in litt. 2016).

\section{Idaea albitorquata (PÜNGELER, 1909)}

Neu für die Ionischen Inseln.

Die Art kommt auf dem Balkan und der Türkei in der subspec. madoniensis HausmanN, 1993 vor. Ab 2000 relativ häufig bei Vathi zwischen dem 25.04. und 12.05. am Licht beobachtet.

\section{Idaea camparia (HERRICH-SCHÄFFER, 1852)}

Eine häufige Idaea-Art auf den Inseln, bereits von Kefalonia durch ReBEL (1910: 424) unter sodaliaria genannt, fehlt bei Hausmann (2004: 170). Емbacher fand die Art auf Zakynthos (Argassi) im Jahre 1998 (in litt. 2016). Funde ab 2000 vom 25.04. bis 13.05 bei Vathi, Exogi, Ormos Kakogilos, Assos und Vliko.

\section{Idaea subsericeata (HAWORTH, 1809)}

Hausmann (2004: 173) bildet Korfu als Fundort ab. Weiterhin am Aviathos lake am 30.04.2003 mittels LF. in 1 Ex. nachgewiesen.

\section{Idaea trigeminata (HAWORTH, 1809)}

Bereits von Rebel (1910: 424), nicht bei HausmanN (2004: 185) dargestellt, sowie EMBACHER (11.-17.07.1986) (in litt. 2016) für Korfu genannt. Weitere Funde bei Assos (3 Ex. am 01.05.2001) und Monastery Taxiarchon (1 Ex. am 11.05.2002) jeweils LF.

\section{Idaea distinctaria (BoIsDUVAL, 1840)}

Hausmann (2004: 213) zeigt die Art von Zakynthos. Vom Autor relativ verbreitet auf Ithaka (Vathi, Exogi), Kefalonia (Assos) und Lefkada (Vliko) zwischen dem 25.04. und 13.05. ab dem Jahr 2000 nachgewiesen.

\section{Idaea ostrinaria (HÜBNER, 1813)}

Mehrfach in der Literatur für Argostoli (REBEL, 1910: 424) und Nidri/Lefkada (REBel, 1932: 54) genannt, fehlt bei Hausmann (2004: 208). Häufig bei Vathi und in Anzahl bei Assos zwischen dem 25.04. und 13.05. (LF.).

\section{Idaea rubraria (STAUDINGER, 1901)}

\section{Neu für die Ionischen Inseln.}

Meist einzeln am Licht bei Vathi ab 2001 zwischen dem 04. und 12.05. und ein Ex. am Ormos Kaminia: 06.05.2015 (alles am Licht).

\section{Idaea straminata (BoRKHAUSEN, 1794)}

STAUDinger (1871: 150) nennt sie bereits von Korfu, fehlt bei Hausmann (2004: 233). Ein Ex. am 15.05.2002 am Ormos Kakogilos am Licht.

\section{Rhodostrophia calabra (Petagna, 1787)}

Schon bei Staudinger (1871: 157) und Rebel (1910: 424) von Korfu und Kefalonia benannt, fehlt bei HausmanN (2004: 376). Von dort neuerdings ebenfalls gemeldet (vergl. Foto, leg. Ginzinger unter www.lepiforum.de). Neue Funde ab 2001 bei Vathi und Vliko (03.05.-12.05. tags und am Licht).

\section{Casilda antophilaria (HÜBNER, 1813)}

Auf Korfu kommt nach Hausmann (2004: 433) die subspec. rosearia Treitschke, 1828 vor und bereits von dort durch BuRESCH \& IltsCHEW (1915: 46) als var. rosearia publiziert. Auch durch StAUdINGER (1871: 168) von der Insel mitgeteilt. Neuerdings am 20.07.2014 bei Lefkada beobachtet (vergl. Foto, leg. Graf unter www.lepiforum.de). 


\section{Orthonama obstipata (FABRICIUS, 1794)}

Rebel (1910: 425, 1912: (14)) verzeichnet die Art für Korfu, bei Hausmann \& Vidialepp (2012: 82) nicht genannt. Vier Einzelfunde dieser Wanderfalterart am Licht: am 01.05.2001 bei Assos, am 03.05.2001 und 11.05.2002 bei Vathi sowie am 07.05.2001 bei Exogi.

\section{Xanthorhoe fluctuata (LINNAEUS, 1758)}

Neu für die Ionischen Inseln.

Mehrere Einzelfunde durch Lichtfang bei Vathi, Exogi, am Aviathos lake und Ormos Kakogilos zwischen dem 06.04. und dem 07.05. ab 2001.

\section{Catorhoe hortulanaria (STAUDINGER, 1879)}

Neu für die Ionischen Inseln.

Die Art wurde von Staudinger nach Ex. aus Kleinasien (= Türkei) beschrieben. Nach Hausmann \& ViIDALEPP (2012: 125) ist sie auch von einigen griechischen Inseln bekannt geworden, westlich bis zur Peloponnes. Der Neufund von Vathi; 1 ㅇ vom 05.05.2003 (LF.) (Fig. 10), stellt nunmehr den westlichsten Nachweis der Art dar (det. HausmanN).

\section{Catarhoe permixtaria (HeRRICH-SCHÄFFER, 1856)}

Neu für die Ionischen Inseln.

Nur im Jahr 2002 nachgewiesen: vom 09. bis 11.05. fünf Ex. bei Vathi, je ein Ex. am 11.05. beim Monastery Taxiarchon und am 13.05.2002 bei Assos (LF.).

\section{Protorrhoe unicata (GUENÉE, 1857)}

Aus der Literatur mit $1 \mathrm{Ex}$. von Argostoli (Rebel, 1910: 425) und 2 o o vom 01.06.1929 von Nidri (Rebel, 1932: 54) bekannt, bei Hausmann \& ViIdAlepP (2012: 120) nicht verzeichnet. Die Art ist auf Kefalonia, Ithaka und Lefkada relativ weit verbreitet und stellenweise häufig: Assos, Livadi, am Aviathos lake, Vathi, Vliko und Katohori jeweils am Licht (30.04.-09.05.).

\section{Earophila badiata (DenIS \& SCHIFFERMüLLER, 1775)}

Zuerst für Griechenland von WeIDLICH (2008: 480) publiziert, fehlt bei HAUSMANN \& ViIDALEPP (2012: 179). Neuerdings von Korfu, Peloponnes und vom Grenzgebiet Nordgriechenland/Mazedonien mitgeteilt (vergl. HausMANN \& VIDIALEPP (2012: 179).

\section{Coenotephria ablutaria (BoIsDuval, 1840)}

Die Art wurde zuerst für Lefkada/Nidri unter Larentia salicata ablutaria BoISDUVAL von ReBEL (1932: 54) genannt. Nach Hausmann \& ViIdalepp (2012: 322) kommt an den Westküsten des Balkans die subspec. probaria bis Korfu vor. Auf dem Peloponnes lebt die subspec. hangayi (Vojnits, 1986), welche sich genetisch nur um 1,6 Prozent (mtDNA) von der probaria unterscheidet (HAUSMANN, 2011: 80). Nachweise der Art liegen von Lefkada (s.o.), Ithaka und Kefalonia vor: Vliko, Vathi, Exogi, Aviathos lake, Agios Eleftherios, Livadi, Monastiráki, Ormos Kakogilos und Konidarata (05.04. bis 05.05.).

\section{Thera variata (DENIS \& SCHIFFERMÜLLER, 1775)}

Hilf fand die Art zuerst bei Argostoli (Rebel, 1910: 425). Es könnte sich jedoch auch um die erst später beschriebene britannica handeln. Dieser Fund fehlt bei Hausmann \& ViIdAlePP (2012: 233); die Populationen in Griechenland gehören der subspec. balcanicola LATTIN, 1951 an. Ein $\sigma^{*}$ erschien am 14.05.2002 bei Agios Eleftherios im Nationalpark „Enos“ am Licht.

\section{Thera britannica (TURNER, 1925)}

Neu für die Ionischen Inseln.

Sympatrisch mit variata vorkommend: Agios Eleftherios ein $\sigma^{*}$ am 14.05.2002 ebenfalls am Licht. HausmanN \& VIIDALEPP (2012: 236) führen als nächstgelegen Fundort die Peloponnes an.

\section{Eupithecia irriguata (HÜBNER, 1813)}

Neu für die Ionischen Inseln.

In Griechenland kommt ausschließlich die subspec. staudingeri ВонАтSCH, 1893 vor, die dunkler und kontrastärmer als die Nominatform ist (Mironov, 2003: 226, 227, Tafel 11). Dieses trifft auch für die 4 Ex., die am 05.04.2005 bei Monastiráki am Licht erschienen zu (det. Mironov).

\section{Eupithecia insigniata (HüBNER, 1790)}

Neu für die Ionischen Inseln.

Zuerst in großer Zahl am Monastery bei Poros am 05.04.2005 festgestellt. Dann erschien ein Ex. am 07./08.04.2005 bei Vathi am Licht.

\section{Eupithecia schiefereri BOHATSCH, 1893}

Mironov (2003: 118) zeigt die Art von Korfu. Jetzt ein neuer Fund auf Kefalonia: 1 Ex. am 04.05.2015 an einer Hauswand in Patrikata. 


\section{Eupithecia dodoneata GUENÉE, 1858}

Neu für die Ionischen Inseln.

Wenige Ex. am Licht ab 2005 auf Ithaka (Vathi), Kefalonia (Ormos Kakogilos, Aviathus lake) und Lefkada (Katohori); Flugzeit vom 06.04. bis 05.05.

\section{Chloroclytis v-ata (HAWORTH, 1809)}

Neu für die Ionischen Inseln.

Mehrfach in Anzahl bei Vathi am Licht zwischen dem 11.07. und 16.07.1993. Später auch mehrfach von DAHL auf Korfu gefunden (24.-25.09.2007, in litt. 2016).

\section{Chesias capriata Prout, 1904}

Rebel (1912: (14)) nennt die Art unter" Chesias spartiata Hrbst." von Korfu. Nach Hausmann \& ViIdAlepp (2012: 477) kommt C. legatella (Denis \& SCHIFFERMÜLLER, 1775) in Griechenland nicht vor. Vielmehr könnte die Angabe auf eine Verwechslung mit C. capriata beruhen, welches dann die einzige Angabe für Griechenland bisher bedeuten würde. C. capricata wurde bisher an der Adriaküste südlich bis Montenegro gefunden (Hausmann \& ViIDALEPP, 2012: 478). Somit wird die Art nicht in die Faunenliste aufgenommen.

\section{Oulobophora internata (PüNGELER, 1888)}

Neu für die Ionischen Inseln.

Ab 2003 in insgesamt 11 Ex. durch LF. am Aviathos lake, Monastiráki, Ormos Kakogilos und Vathi nachgewiesen (05. bis 30.04.). Dies sind die bisher westlichsten Funde in Europa.

\section{Notodontidae}

\section{Drymonia ruficornis (HufNAGEL, 1766)}

Neu für die Ionischen Inseln.

Ab 2002 recht verbreitet und teilweise i.M. am Licht festgestellt: Agios Eleftherios, Aviathos lake, Monasteraki, Ormos Kakogilos und Vathi (LF.) (05.04.-30.04.).

\section{Noctuidae}

In der Tabelle wird wesentlich auf HACKer (1989) zurückgegriffen, da hier eine monografische Bearbeitung vorliegt. Alte Quellen werden nur ausnahmsweise geführt, z.B. wenn sie in der Verbreitungskarte von HACKER fehlen, währenddessen die jüngeren Nachweise differenziert genannt werden.

\section{Acronicta psi (LINNAEUS, 1758)}

Neu für die Ionischen Inseln.

Die weit verbreitete Art wurde ab 2000 auch auf Ithaka festgestellt. Im Zeitraum vom 25.04. bis 17.05. flog die Art relativ häufig ans Licht; auch am Monastery Taxiarchon. Die Ex. sind relativ groß, auffällig kräftig gezeichnet, insbesondere das Psi-Zeichen und die Wurzelstrieme.

\section{Pechipogo plumigeralis (HÜBNER, 1825)}

Zuerst von MaLICKy (1992: 402) mit einem ơ am 27.05.1977 von Argasi (Zakynthos) gemeldet. Je ein ? am 04.05.2001 und am 10.05.2002 bei Vathi am Licht.

\section{Catocala eutychea TREITSCHKE, 1835}

Die Art wurde von Treitschke nach Ex. von Korfu beschrieben (Staudinger, 1871: 139), Kattioulas \& KoutsafikTIS (1977: 119) nennen sie für Kythira und von Hacker (1989: 375, Korfu fehlt in der Verbreitungskarte) wird die Art für Griechenland als allgemein verbreitet genannt. Später von EMBACHER auch auf Korfu gefunden (in litt. 2016). Ebenfalls von Lefkada bekannt (Malicky, 1992: 401 und Piatkowski, 2006: 354). Malicky (1992: 401) führt mehrere Fundorte auf Kefalonia an: Sami, Kulurata und die Osthänge des Enos. Die Flugzeit beginnt Anfang Mai und danach ist die Art eine der häufigsten Eulenfalter, die am Tage an Felsen und aus dem Gebüsch aufgescheucht wurden. Am Licht teilweise sehr häufig; am Ormos Kakogilos am 15.05.2002 über 90 Ex. in der Lichtfalle. Auf Ithaka bei Stavros und Vahti festgestellt. Auch von Krumm auf Korfu gefunden (vergl. Foto unter www.lepiforum.de).

\section{Grammodes bifasciata (PetAgna, 1786)}

Neu für die Ionischen Inseln.

Relativ selten in Griechenland beobachtet. Das erste Ex. erschien am 05.05.2001 bei Vathi am Licht. Piatkowski (2006: 355) nennt Funde wenig später zwischen dem 20. und 25.09.2001 von Evgiros auf Lefkada.

\section{Catephia alchymista (DenIs \& SchiffermülleR, 1775)}

Zuerst von Piatkowski (2006:356) auf Lefkada zwischen dem 22. und 26.05.2000 nachgewiesen. Dann jeweils Einzelfunde am 02.05.2001, 07.05.2001 und 10.05.2002 am Licht bei Vathi. Am 13.05.2002 erschien ein weiteres Ex. bei Assos am Licht. 


\section{Araeopteron ecphaea (HAMPSON, 1914)}

\section{Erstfund für Europa.}

Offenbar zuerst für Europa von DAHL nachgewiesen, welcher ein Ex. am Robolla Beach nahe Roda auf Korfu am 25.09.2007 fand und fotografierte (Fig. 11) (vergl. http://www.lepiforum.de/lepiwiki.pl?Araeopteron_ Ecphaea). Die Art wurde aus Nigeria/Faro beschrieben. Obwohl sie noch bei KARSHOLT \& RAzOwsKI (1996) fehlt hat sie sich inzwischen im südeuropäischen Mittelmeerraum angesiedelt. Nach der [Fauna Europaea] kommt die Art in Spanien, den Balearen, Italien, Sizilien, auf Malta und in Griechenland einschließlich Kreta vor (vergl. www.lepiforum.de).

\section{Zebeera falsalis (HERRICH-SchäFFER, 1839)}

Neu für die Ionischen Inseln.

Zuerst bei Vathi am 09.05.2002 nachgewiesen. Insgesamt 8 Ex. dann bis zum 12.05. im Jahre 2002 und 05.-12.05.2003 nachgewiesen. Ein Ex. erschien am 13.05.2002 bei Assos am Licht. Ein weiteres Ex. noch am 06.05.2015 am Ormos Karini (FL.). HACKER (1989: 342) führt die nächsten Fundorte aus dem Westen der Peloponnes an.

\section{Thysanoplusia circumscripta (FreYER, 1831)}

Neu für die Ionischen Inseln.

Das erste Ex. für die Ionischen Inseln wurde durch LF. am 07.05.2001 bei Exogi nachgewiesen. Ein weiteres Ex. bei Livadi am Licht (01.05.2003). Auf dem Peloponnes offenbar weiter verbreitet und nicht selten (vergl. HACKER, 1989: 366) und war deshalb auch hier zu erwarten.

\section{Glossodice polygramma (DUPONCHEL, 1842)}

Neu für die Ionischen Inseln.

Zuerst am 11. und 12.05.2002 bei Vathi (Ithaka) (3 Ex.) aufgefunden. Ausserdem ein Ex. am 11.05.2002 am Monastery Taxiarchon, weitere 3 Ex. am 13.05.2002 bei Assos und ein Ex. am 15.05.2002 am Ormos Kakogilos (LF, FL.).

\section{Metachrostis velox (HÜBNER, 1813)}

Neu für die Ionischen Inseln.

DAHL beobachtete ein Einzelexemplar am 24.09.2007

(Robolla Beach bei Roda, Korfu, in litt. 2016).

\section{Cucullia blattariae (ESPER, 1790)}

Neu für die Ionischen Inseln.

Ein Einzelnachweis am 25./26.04.2000 bei Vathi am Licht.

\section{Calophasia platyptera (ESPER, 1788)}

Neu für die Ionischen Inseln.

Ein Ex. erschien am 30.04.2003 am Aviathos lake am Licht. Von Lefkada meldet Piatkowski (2006: 363) ebenfalls die Art (31.05.-03.06.2003).

\section{Omphalophana anatolica (LEDERER, 1857)}

Neu für die Ionischen Inseln.

Hauptsächlich auf Ithaka (Umg. Vathi, Monastery Taxiarchon) zwischen dem 25.04. und 03.05. (10 Ex. 2000 und 2003) festgestellt. Auch auf Lefkada (Vliko) in einem Ex. am 08.05.2002 und auf Kephalonia am Aviathos lake in einem Ex. am 04.05.2015 nachgewiesen (alles LF.).

\section{Amephana dalmatica (ReBEL, 1919)}

Neu für die Ionischen Inseln.

Ab 2000 vom 25.04. bis 05.05. am Licht beobachtet: Vathi (5 Ex.) und Livadi (3 Ex.).

\section{Caradrina wullschlegeli PüngeLER, 1903}

Neu für die Ionischen Inseln.

Die Populationen des Balkans und Kleinasiens gehören der subspec. schwingenschussi (Boursin, 1936) an. Nach HACKer (1989: 300, 421; 1996a: 256) nur wenige Funde in Griechenland, jetzt ein Ex. am 25.-26.04.2000 bei Vathi (LF.).

\section{Caradrina flavirena (GUENEE, 1852)}

Neu für die Ionischen Inseln.

Ab 2000 mehrfach am Licht bei Vathi, Agios Eleftherios, Ormos Kakogilos und Livadi zwischen dem 25.04. und 07.05. Auch von Piatkowski (2006: 364) für Lefkada aufgeführt.

\section{Hoplodrina superstes (OCHSENHEIMER, 1816)}

Neu für die Ionischen Inseln.

In der Umg. von Katohori befanden sich am 22.04.2012 drei Ex. in der FL. HACKER (1989: 291; 1996a: 255) listet alle griechischen Funde auf mit einer Flugzeit von Mitte Juli bis Anfang September. In tieferen Lagen vermutet er zwei Generationen, welches mit den frühen Funden im April durchaus bestätigt werden kann. 


\section{Hoplodrina ambigua (DENIS \& SCHIFFERMüLLER, 1775)}

Neu für die Ionischen Inseln.

Durch LF. mehrfach nachwiesen ab dem Jahre 2000; so bei Vathi, Monastery Taxiarchon, Ormos Kaminia, Vliko, Assos und am Ormos Kakogilos (26.04.-15.05.).

\section{Charanycha trigrammica (HufnageL, 1766)}

Neu für die Ionischen Inseln.

In Griechenland relativ wenig beobachtet, vergl. HACKER (1989: 289). Bei Vliko am 09.05.2002 fand sich die Art i.M. und am 22.04.2012 mit 6 Ex. in der FL.

\section{Chloantha hyperici (DenIS \& SCHIFFERMülLER, 1775)}

Neu für die Ionischen Inseln.

In Griechenland weit verbreitet. Der erste Nachweis stammt vom 03.05.2001 am Licht bei Vathi und dort auch weitere Ex. vom 03.-04.05.2003. Ausserdem ein Ex. am Aviathos lake am 30.04.2003 und 2 Ex. bei Livadi am 01.05.2003, jeweils am Licht. Piatkowski (2006: 370) nennt sie von Lefkada.

\section{Conistra vaccinii (LINNAEUS, 1761)}

Neu für die Ionischen Inseln.

In Mitteleuropa weit verbreitet und häufig, wird jedoch nach Süden immer seltener, wie schon HACKER (1989: 195) bemerkt. Nur zwei Einzelstücke bei Monastiráki am 05.04.2005 und am Ormos Kakogilos am 06.04.2005 beobachtet (LF.).

\section{Lithophane ledereri (STAUDINGER, 1892)}

Neu für die Ionischen Inseln.

Je ein Ex. beim Lichtfang am 30.04.2003 am Aviathos lake und Monastiráki am 05.04.2005. Bisher nur wenig aus Griechenland bekannt (vergl. HACKer, 1989: 170). Der Autor fand die Art mehrfach 2001 bis 2007 im Notia Pindos (Weidlich, 2008: 486) und 2002 auf dem Peloponnes im Taigetos-Gebirge (Weidlich, unveröffentlicht) sowie 2006 bis 2010 in der Zagoria des Voria Pindos (WeIdLich, 2012b: 1250).

\section{Abromia sicula (TURATI, 1909)}

Neu für die Ionischen Inseln.

Nach Hacker (1989: 256) kommt in Griechenland die subspec. syriaca (Osthelder, 1933) vor. Zuerst in Anzahl am 02.05.2001 bei Vathi, später dort i.A. und auch am 08.05.2002 (häufig). Weiterhin am 11.05.2002 und am Monastery Taxiarchon (i.A.) nachgewiesen. Ein
Ex. erschien am 14.05.2002 bei Agios Eleftherios, ein weiteres Ex. am 30.04.2003 am Aviathos lake sowie am 22.04.2012 bei Vliko (LF., FL.).

\section{Oligia latruncula (DeNIS \& SCHIFFERMÜLLER, 1775)}

Neu für die Ionischen Inseln.

Jeweils ein Einzelstück am 10.05.2002 bei Vathi, am 11.05.2002 in der Lichtfalle beim Monastery Taxiarchon und am 15.05.2002 am Ormos Kakogilos am Licht. In Griechenland nur wenig verbreitet (vergl. HACKER, 1989: 268).

\section{Hada plebeja (LINNAEUS, 1761)}

Nach Staudinger (1871: 123) schon von Erber auf Korfu gefunden. Fehlt bei HaCker (1989: 92).

\section{Hecatera bicolorata (HufNAGEL, 1766)}

Neu für die Ionischen Inseln.

Das erste Ex. wurde am 08.05.2002 auf Lefkada bei Vliko gefangen. Im darauffolgenden Jahr ein weiteres Ex. auf Ithaka bei Vathi (04.05.2003). PiATKowski (2006: 375) berichtet ebenfalls über ein Vorkommen bei Evgiros auf Lefkada (31.05.-03.06.2003).

\section{Conisania luteago (DenIS \& SCHIFFERmüLleR, 1775)}

Neu für die Ionischen Inseln.

Ein Ex. am 11.05.2002 in der Lichtfalle beim Monastery Taxiarchon, ein weiteres Ex. am 30.04.2003 am Aviathos lake sowie ein Ex. am 04.05.2003 bei Vathi am Licht.

\section{Hadena adriana (SCHAWERDA, 1921)}

Neu für die Ionischen Inseln.

1 o am Licht bei Vathi 25.04.2000. In Griechenland nur wenig beobachtet (HACKER, 1989: 110; 1996b: 154) und der nächste Fundort befindet sich auf der Peloponnes (vergl. Fotos, leg. Friedrich unter www.lepiforum.de).

\section{Hadena magnolii (BoISDUVAL, 1829)}

\section{Neu für die Ionischen Inseln.}

Je ein Ex. am 25.04.2000 bei Vathi (LF.) und am 26.04.2000 bei Stavros tags an einer Felswand. Zwei Ex. am 10.05., ein $o^{t}$ am 12.05.2002 bei Vathi (LF.) und 8 Ex. am 11.05.2002 beim Monastery Taxiarchon in der Lichtfalle. Die Ex. der Insel sehen kontrastreicher und bunter aus, als die vom griechischen Festland. 


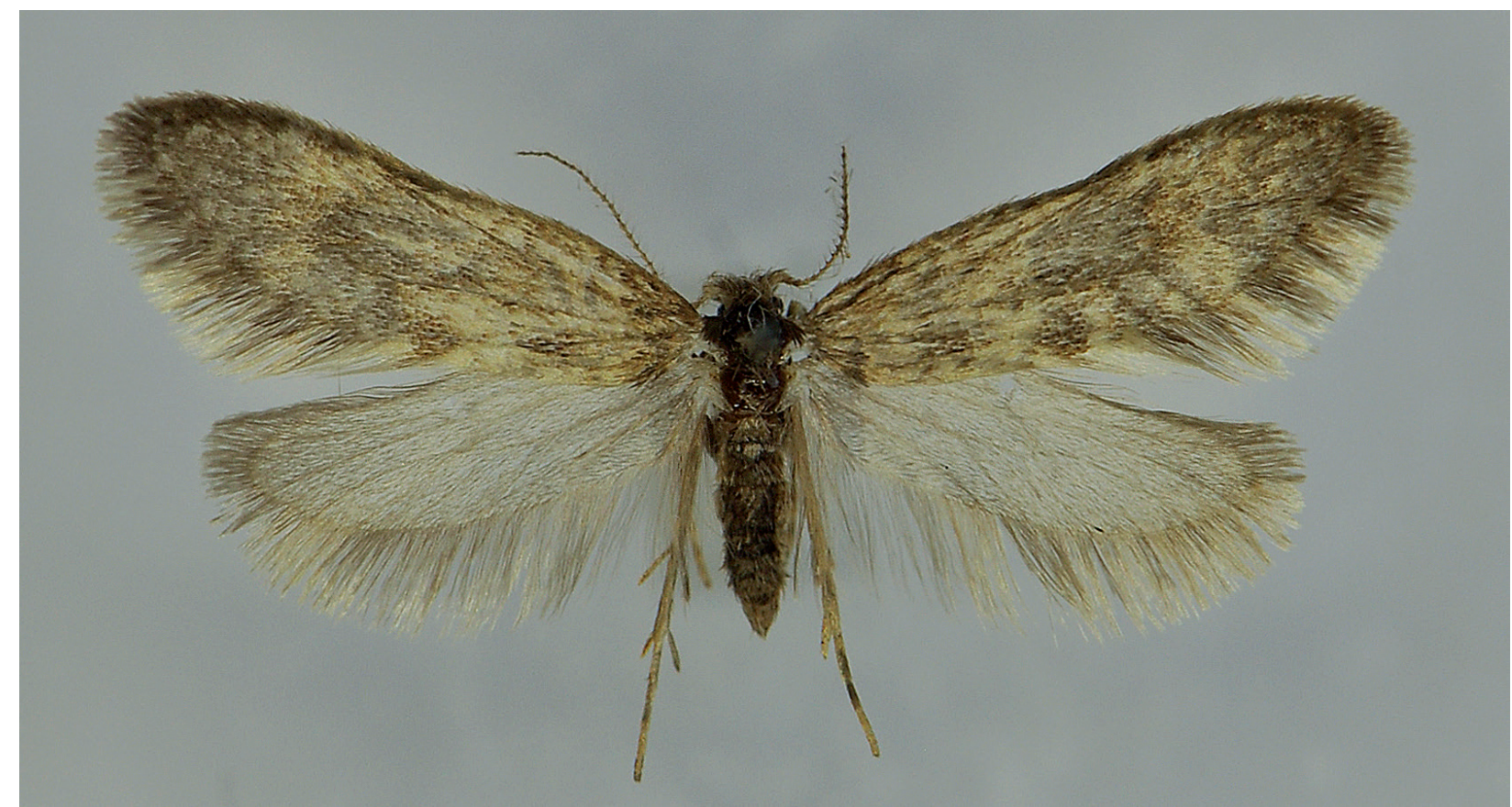

Fig. 1: Bankesia cephalonica spec. nov., Holotypus: $0^{\star}, 02.05 .2003$ (Flügelspanne 13,0 mm): Europa meridionalis, Greece-West/ Kefalonia, Ionische Inseln, Nationalpark Enos-Gipfel, 1.600 m NN, leg. Dr. M. WeidLich (Foto: M. Weidlich).

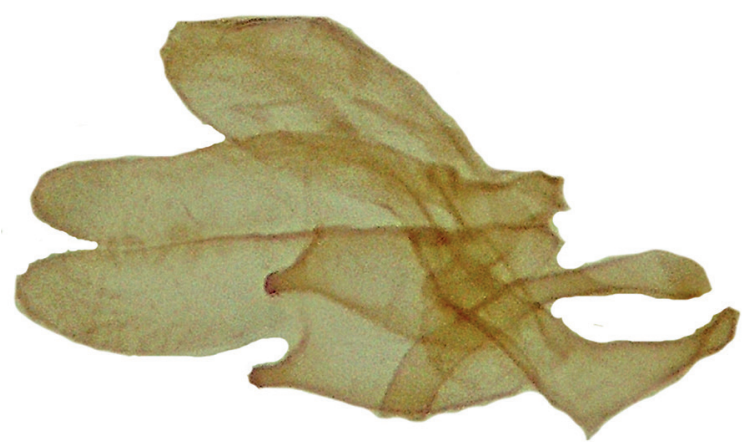

Fig. 2: Bankesia cephalonica spec. nov., Paratypus: $\sigma^{\star}, 02.05 .2003$, Genital, Lateralansicht: Europa meridionalis, Greece-West/ Kefalonia, Ionische Inseln, Nationalpark Enos-Gipfel, $1.600 \mathrm{~m} \mathrm{NN}$, leg. Dr. M. Weidlich (Foto: W. Arnscheid).

Fig. 3: Bankesia cephalonica spec. nov., Paratypus: ㅇ, e.o. 25.01.2016: Europa meridionalis, Greece-West/Kefalonia, Ionische Inseln, Nationalpark Enos-Gipfel, $1.600 \mathrm{~m} \mathrm{NN}$, leg. Dr. M. Weidlich.

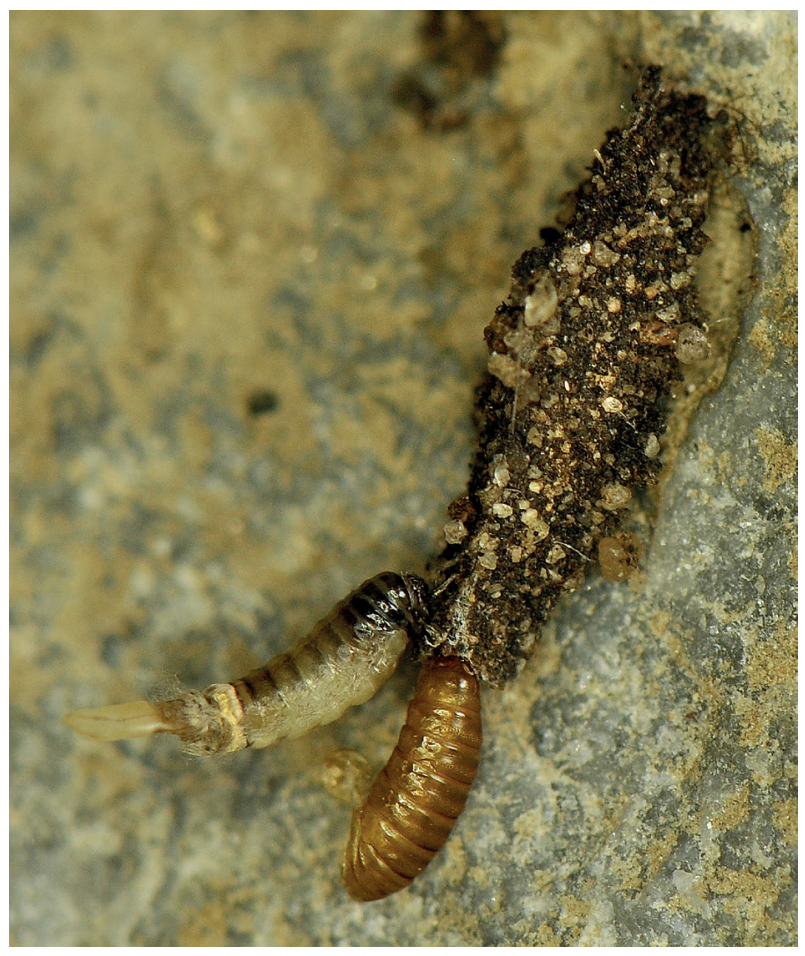

TTPSY007-08|Bankesia_conspurcatella_England TTPSY008-08|Bankesia_conspurcatella_England

LEEUA045-11|Bankesia_conspurcatella_Denmark _ PHLAl1019-14|Bankesia_cephalonica_Greece -TIPSY496-12|Bankesia_montanella_France_Corse PHLAH322-12|Pseudobankesia_lichenaria_Greece -POESE092-15]Dahlica_lichenella_Germany

$$
\longmapsto .05
$$

Fig. 4: Genetische Unterschiede zu B. conspurcatella und B. montanella in der Darstellung als Distanzmatrix $(1,0=100 \%)$ gemäß Tab. 2. 


\section{Hadena perplexa (DENIS \& SCHIFFERMÜLLER, 1775)}

Neu für die Ionischen Inseln.

Ein 우 am Licht bei Assos am 01.05.2001.

\section{Hadena syriaca (OSTHELDER, 1933)}

Neu für die Ionischen Inseln.

Lediglich ein Ex. durch LF. am 08.05.2002 bei Vliko.

\section{Mythimna alopecuri (BoIsduval, 1840)}

Neu für die Ionischen Inseln.

HACKer (1989: 134) publiziert den Erstfund für Griechenland von Delfi. Nun erschien das erste Ex. auf den Ionischen Inseln: ein + am 02.05.2001 bei Vathi am Licht. Neuerdings auch von Grevena gemeldet (siehe Foto, leg. PAPÉ unter www.lepiforum.de).

\section{Mythimna riparia (RAMBUR, 1829)}

Neu für die Ionischen Inseln.

Ein Ex. am 29.09.2007 am Hauslicht am Rabolla Beach bei Roda (Korfu) (siehe Foto, leg. DAHL unter www. lepiforum.de und in litt. 2016).

\section{Mythimna unipuncta (HAWORTH, 1809)}

Neu für die Ionischen Inseln.

Die ersten Funde stammen vom 03.05.2001, es kamen 2 Ex. ans Licht bei Vathi.

Später nicht wieder beobachtet. PiatKowski (2006: 378) meldet die Art von Lefkada (20.-25.09.2001).

\section{Orthosia incerta (HUFNAGEL, 1766)}

Neu für die Ionischen Inseln.

Am 05.04.2005 i.M. bei Monastiráki am Licht.

\section{Orthosia miniosa (DenIS \& SCHIFFERmülleR, 1775)}

Neu für die Ionischen Inseln.

Je ein Einzelstück am 30.04.2003 am Aviathos lake (LF.) und am 22.04.2012 bei Katohori (FL.). Nach HACKER (1989: 122) nur wenig in Griechenland beobachtet, dort 4 Fundorte verzeichnet.

\section{Orthosia cerasi (FABRICIUS, 1775)}

Neu für die Ionischen Inseln.
Nachgewiesen für Ithaka (zuerst 2000) und Kefalonia, stellenweise häufig bis zu 40 Ex. am Licht: Vathi, Aviathos lake, Monastiráki, Ormos Kakogilos (05.04. bis 30.04.).

\section{Orthosia dalmatica (WAGNER, 1909)}

Neu für die Ionischen Inseln.

Ein Einzelstück kam am 30.04.2003 am Aviathos lake ans Licht. Bei HACKer (1989) noch nicht für Griechenland angegeben, erst später dann von mehreren Autoren gemeldet.

\section{Noctua pronuba (LINNAEUS, 1758)}

Neu für die Ionischen Inseln.

Mehrfach bei Assos (zuerst am 01.05.2001), Ormos Kakogilos, Vathi, Gerahori (tags), Lefki (tags), Monastery Taxiarchon, Ormos Kaminia, Vliko zwischen dem 30.04. und dem 15.05. am Licht. Piatkowski (2006: 379) nennt sie ebenfalls von Lefkada.

\section{Agrotis ipsilon (HufNAGEL, 1766)}

Neu für die Ionischen Inseln.

Ab 2001 mehrfach zwischen dem 02.05. und 13.05. durch Lichtfang bei Vathi, Monastery Taxiarchon, Ormos Kaminia, Assos und am Aviathos lake nachgewiesen. Auch auf Lefkada durch PiATKowski (2006: 382) nachgewiesen.

\section{Agrotis spinifera (HÜBNER, 1808)}

Neu für die Ionischen Inseln.

DAHL beobachtete und fotografierte ein Ex. am 29.09.2007 am Robolla Beach bei Roda (Korfu) (in litt. 2016 sowie im www.lepiforum.de).

\section{Lymantridae}

\section{Ocneria rubea (DenIS \& SCHIFFERMüLLER, 1775)}

Neu für die Ionischen Inseln.

Ebenfalls von DAHL am 24.09.2007 bei Roda, Robolla Beach (Korfu) gefunden (in litt. 2016 und vergl. Foto unter www.lepiforum.de).

\section{Nolidae}

\section{Meganola togatulalis (HÜBNER, 1798)}

Im Jahr 2000 und 2003 bei Evgiros (Lefkada) nachgewiesen (De Freina \& Piatkowski, 2006: 246). Weiterhin 
1 Ex. am 10.05. und 2 Ex. am 11.05.2002 bei Vathi und 1 Ex. am 11.05.2002 beim Monastery Taxiarchon (Ithaka) am Licht.

\section{Nola cicatricalis (TREITSCHKE, 1835)}

Neu für die Ionischen Inseln.

Ein Einzelstück erschien am 05.04.2005 bei Monastiráki am Licht.

\section{Nola subchlamydula StaUdingeR, 1871}

Aus „Attica“ von Staudinger (1871: 108) beschrieben und später von ReBel (1924: 42) nach Funden von STERNECK aus dem Jahre 1910 von Korfu vermerkt. In den letzten Jahren auch auf Lefkada (Evgiros) im Jahr 2001 (De Freina \& Piatkowski, 2006: 247) und Kefalonia festgestellt: am 30.04.2003 erschienen 8 Ex. am Aviathos lake am Licht.

\section{Nycteola revayana (ScOPOLI, 1772)}

Neu für die Ionischen Inseln.

Ein Einzelstück erschien am 06.04.2005 am Ormos Kakogilos am Licht.

\section{Nycteola columbana (TURNER, 1925)}

Neu für die Ionischen Inseln.

Durch Lichtfang bei Vathi am 09.05.2002 (1 Ex.), bei Assos am 13.05.2002 (2 Ex.) und am Ormos Kakogilos am 15.05.2002 (1 Ex.) nachgewiesen.

\section{Arctiidae}

\section{Eilema depressa (ESPER, 1787)}

Bereits von Staudinger (1871: 109) von Korfu nach Rogenhofer genannt. Neuerdings ein Ex. bei Assos am 01.05.2001, später 3 Ex. am 15.05.2002 am Ormos Kakogilos, alle Falter am Licht.

\section{Eilema costalis (ZeLLER, 1847)}

Neu für die Ionischen Inseln.

Verbreitet und nicht selten auf Ithaka (Vathi, Exogi, Monastery Taxiarchon), Lefkada (Vliko) und Kefalonia (Assos, Livadi). Die Art wurde ab 2000 im Zeitraum vom 25.04. bis 12.05. beobachtet (LF).

\section{Amata marjana (STAUDER, 1913)}

Neu für die Ionischen Inseln.

Ein frisch geschlüpftes Ex. am 14.05.2002 bei Magganos.

\section{Dysauxes famula (FrEYER, 1836)}

StAudinger (1871: 106) gibt für Korfu „Naclia Punctata F." an. Nach aktuellem Kenntnisstand kommen Dysauxis punctata (FABricIUs, 1781) wie auch Dysauxis ancilla (LinnaEus, 1767) nicht in Griechenland vor (De Freina \& Witt, 1987: 194, 195, 632, Fibiger et al., 2011: 215, 216). Köstler (1991: 102 und Hund, 2002, vergl. Foto unter www.lepiforum.de) bestätigen dann das Vorkommen von D. famula auf Korfu. Ein Ex. erschien am 12.05.2002 bei Vathi und 3 Ex. am Ormos Kakogilos am 15.05.2002 am Licht.

\section{Utetheisa pulchella (LINNAEUS, 1758)}

Neu für die Ionischen Inseln.

DAHL beobachtete am 30.09.2007 ein Ex. am Robolla Beach, Roda auf Korfu (in litt. 2016).

\section{Spilosoma lubricipeda (LINNAEUS, 1758)}

Neu für die Ionischen Inseln.

Mehfach von DAHL am 24. und 25.09.2007 am Robolla Beach, Roda auf Korfu nachgewiesen (in litt. 2016).

\section{Arctia festiva (HufNAGEL, 1766)}

Staudinger (1871: 111) nennt die Art von Kefalonia. Der Autor beobachtete ein Ex. (05.05.2005) bei Monastiráki durch LF. und 2 Ex. am frühen Tage (05.05.2015) in der Vegetation bei Agios Eleftherios.

\subsection{Systematisches Verzeichnis (Trichoptera)}

In seiner Griechenlandfauna behandelt MALICKY (2005) auch umfassend die Köcherfliegenfauna der Ionischen Inseln. Darin gibt er für Korfu 29 Arten, für Lefkada14 Arten, für Kefalonia 23 Arten, für Zakynthos 8 Arten und für Kythira 9 Arten an (MALICKy, 2005: 34, 35). Für Ithaka werden keine Angaben gemacht, obwohl hier an der PlathitryasQuelle sicherlich eine spärliche Köcherfliegenfauna zu vermuten ist. Auf Korfu lebt eine endemische Trichopterenart: Hydropsyche perseus MALICKY, 2001 (MALICKY, 2005: 34, 101, 198).

Bei seinen Aufsammlungen brachte der Autor auch Material von Lefkada und vor allem von Kefalonia aus 


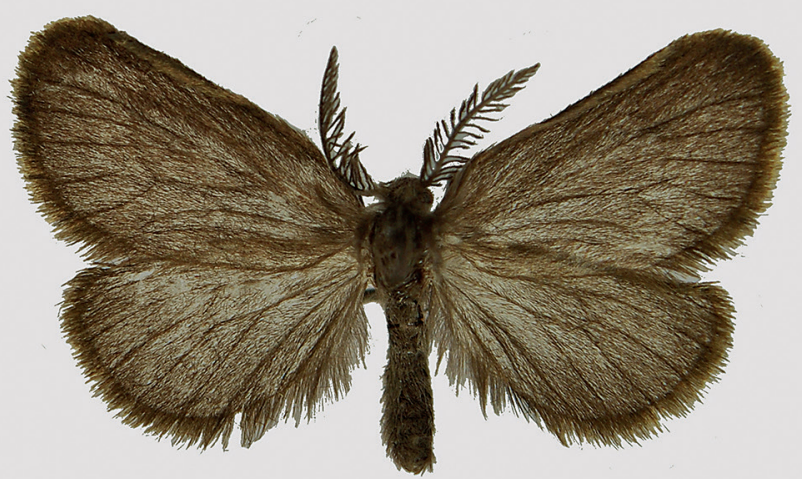

$\mathbf{a}$

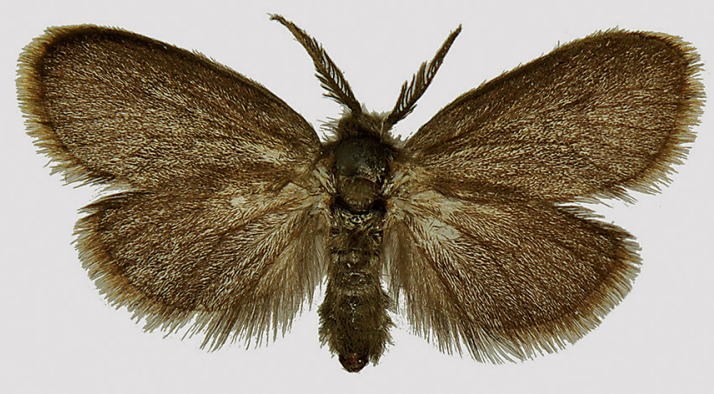

c

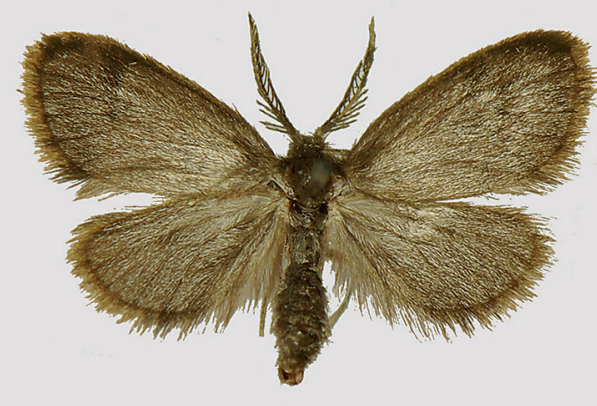

b

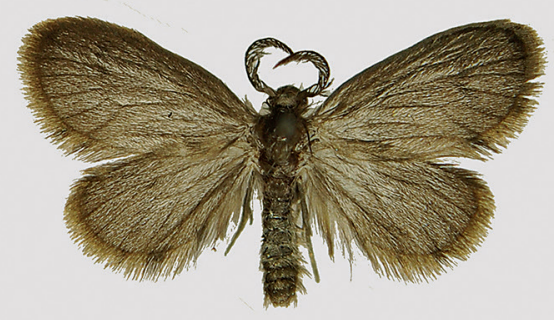

d

Fig. 5: $\sigma^{\star}$. a Reisseronia ionica spec. nov., Holotypus: e.l. 21.05.2005: Europa meridionalis, Greece-West/Kefalonia, Ionische Inseln, Skoloupas, Myrto Bay, $300 \mathrm{~m}$ NN, leg. Dr. M. Weidlich (Flügelspanne: $12 \mathrm{~mm}$ ). - b Reisseronia ionica odysseus subspec. nov., Holotypus: e.l. 29.05.2003: Europa meridionalis, Greece-West/Ithaka, Ionische Inseln, Umg. Lefki, 3 km S, 200-300 m NN, leg. Dr. M. Weidlich. - c Reisseronia magna ơ : e.l. 27.07.1985, Europa meridionalis, Greece, Peloponnes, Monemvasia/Lakonia, leg. G. Christensen, cult. M. Weidlich. - d Reisseronia ionica lefkadensis subspec. nov., Holotypus: e.o.15.05.2013: Europa meridionalis, Greece-West/Lefkada, Ionische Inseln, Katohori. 150 m NN., leg. Dr. M. WeIdLICH (alle Fotos M. WeIdLICH).

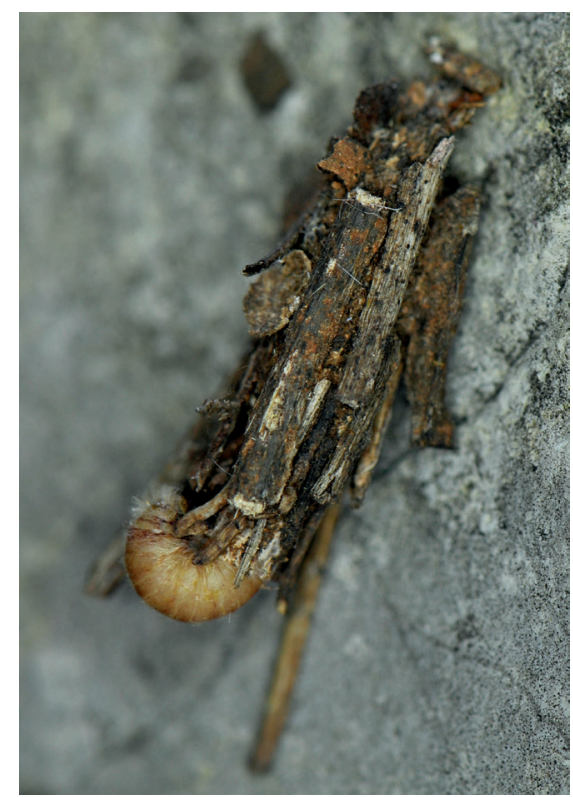

Fig. 6: Reisseronia ionica odysseus subspec. nov., Paratypus: o , e.l. 05.06.2015: Europa meridionalis, Greece-West/ Ithaka, Ionische Inseln, Umg. Lefki $3 \mathrm{~km} \mathrm{S,} \mathrm{200-300} \mathrm{m,} \mathrm{leg.}$ Dr. M. Weidlich (Foto: M. WeIDlich).

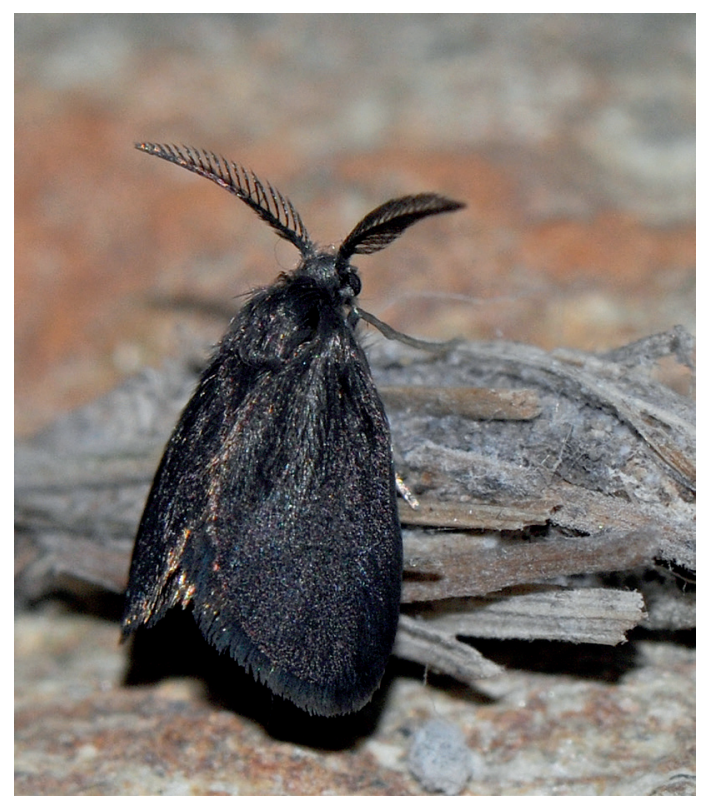

Fig. 7: Reisseronia ionica lefkadensis subspec. nov., Paratypus: $\sigma^{\star}$, e.o. 23.05.2013: Europa meridionalis, Greece-West/Lefkada, Ionische Inseln, Umg. Katohori, 150 m, leg. Dr. M. WeIdLICH (Foto: M. WeIdLICH). 


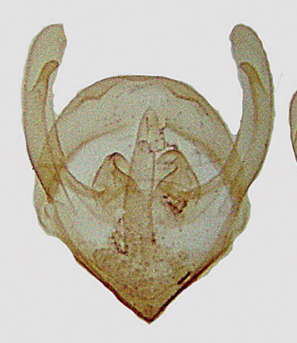

$\mathbf{a}$

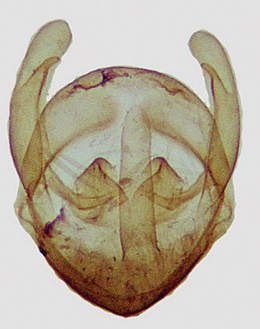

d

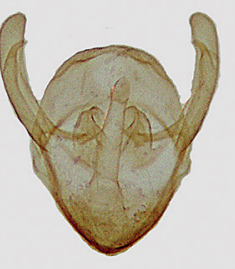

b

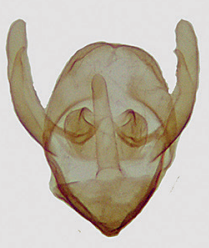

c

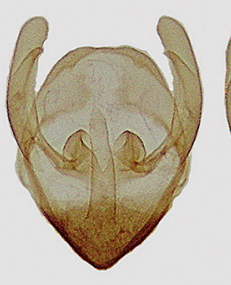

e

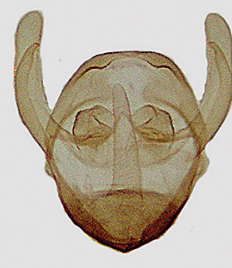

f
Fig. 8: $0^{\top}$ - Genital. - a Reisseronia ionica spec. nov., Paratypus: e.l. 03.05.2005: Europa meridionalis, Greece-West/Kefalonia, Ionische Inseln, Skoloupas, Myrto Bay, 300 m NN. - b Reisseronia ionica odysseus subspec. nov., Paratypus: e.p. 17.05.2002: Europa meridionalis, Greece-West/Ithaka, Ionische Inseln, Umg. Exogi unterh., 300 m. - c Reisseronia ionica lefkadensis subspec. nov., Paratypus: e.p. 10.05.2003: Europa meridionalis, Greece-West/Lefkada, Ionische Inseln, Umg. Katohori, $150 \mathrm{~m}$. - d Reisseronia ionica spec. nov., Paratypus: e.p. 05.05.2015: Europa meridionalis, Greece-West/Kefalonia, Ionische Inseln, Umg. Livadi $2 \mathrm{~km} \mathrm{~N}, 150 \mathrm{~m} \mathrm{NN}$. - e Reisseronia ionica odysseus subspec. nov., Paratypus: e.p. 05.05.2005: Europa meridionalis, Greece-West/Ithaka, Ionische Inseln, Umg. Lefki $3 \mathrm{~km} \mathrm{~S}$, 200-300 m. - f Reisseronia ionica lefkadensis subspec. nov., Paratypus: e.l. 26.05.2013: Europa meridionalis, Greece-West/ Lefkada, Ionische Inseln, Umg. Katohori, $150 \mathrm{~m}$, alles leg. Dr. M. Weidlich (alle Fotos W. R. Arnscheid).

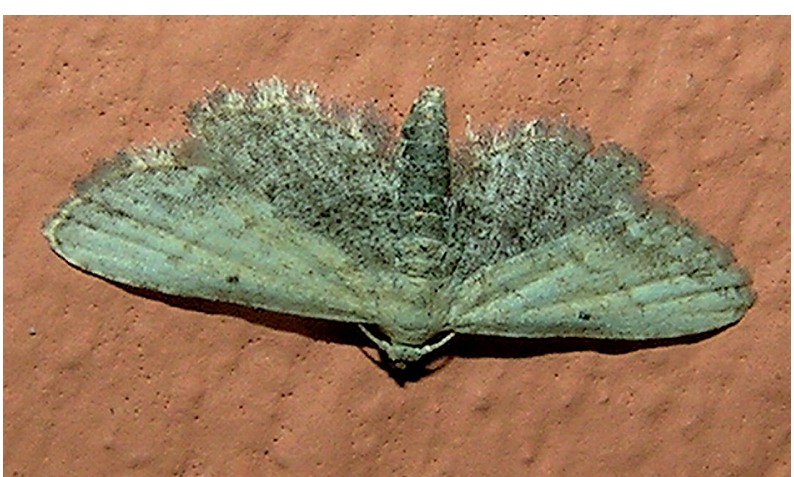

Fig. 11: Araeopteron ecphaea (Hampson, 1914), Robolla Beach nahe Roda auf Korfu, Erstfund für Europa (leg. und Foto vom 25.09.2007, DAHL).

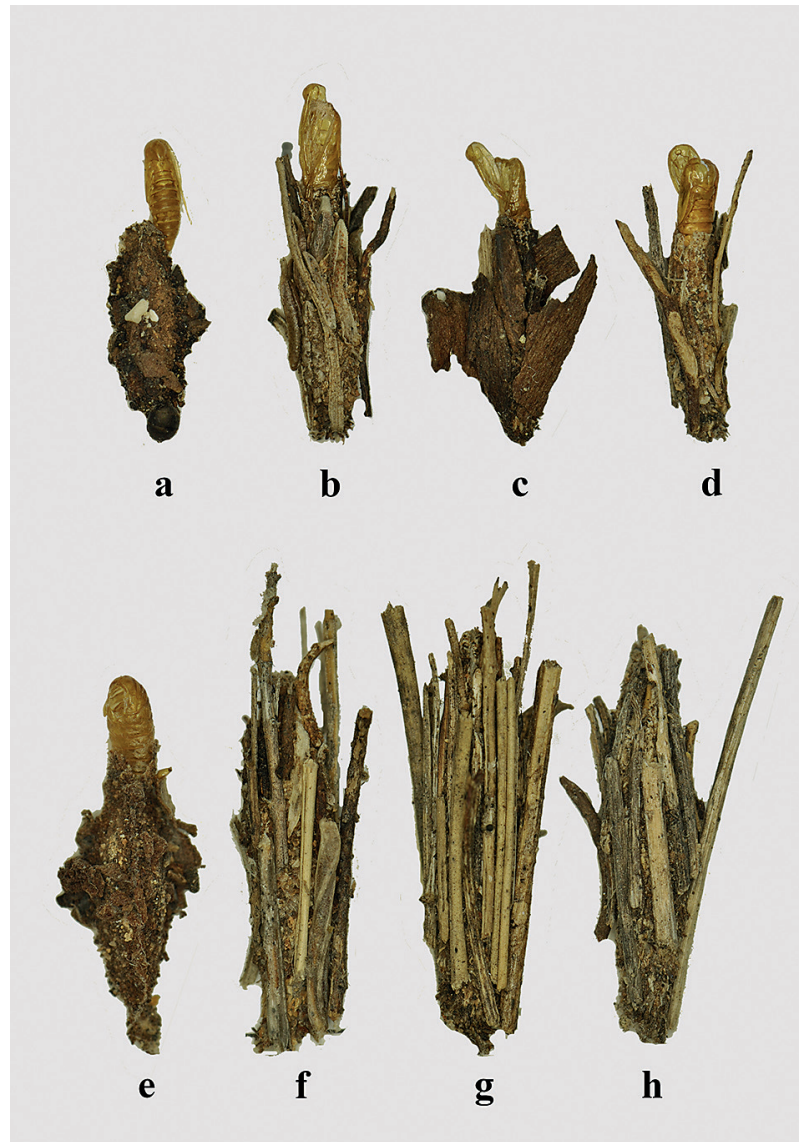

Fig. 9: Säcke. - a Bankesia cephalonica spec. nov., Paratypus:

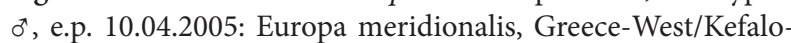
nia, Ionische Inseln, Nationalpark Enos-Gipfel, $1.600 \mathrm{~m} \mathrm{NN}$, (Sacklänge ohne Puppenhülle: 7,7 mm). - b Reisseronia ionica spec. nov., Paratypus: $\sigma^{\star}$, e.p. 16.05.2002: Europa meridionalis, Greece-West/Kefalonia, Ionische Inseln, Skoloupas, Myrto Bay, $300 \mathrm{~m} \mathrm{NN}$. - c Reisseronia ionica odysseus subspec. nov., Paratypus: $\sigma^{*}, 03 .-06.05 .2001$ : Europa meridionalis, GreeceWest/Ithaka, Ionische Inseln, Umg. Lefki $3 \mathrm{~km} \mathrm{S,} \mathrm{200-300} \mathrm{m,}$ - $\mathrm{d}$ Reisseronia ionica lefkadensis subspec. nov., Paratypus: ${ }^{\star}$, 08.05.2002: Europa meridionalis, Greece-West/Lefkada, Ionische Inseln, Umg. Vliko, $70 \mathrm{~m}$. - e Bankesia cephalonica spec. nov., Paratypus: क , 04.04.2005: Europa meridionalis, Greece-West/Kefalonia, Ionische Inseln, Nationalpark EnosGipfel, 1.600 m NN. - f Reisseronia ionica spec. nov., Paratypus: , 16.05.2002: Europa meridionalis, Greece-West/Kefalonia, Ionische Inseln, Skoloupas, Myrto Bay, 300 m NN. - g Reisseronia ionica odysseus subspec. nov., Paratypus: ㅇ, 03.-06.05.2001: Europa meridionalis, Greece-West/Ithaka, Ionische Inseln, Umg. Lefki 3 km S, 200-300 m. - h Reisseronia ionica lefkadensis subspec. nov., Paratypus: 9 , 05.05.2003: Europa meridionalis, Greece-West/Lefkada, Ionische Inseln, Umg. Katohori, 150 m, alles leg. Dr. M. Weidich (alle Fotos: M. WeidLich). 
den Jahren 2003 (ausgewertet bei MaLICKy, 2005) und 2015 als Beifänge mit. Die Untersuchungen ergab ein Spektrum von 10 Arten (alles det. MEY).

\section{Polycentropodidae}

\section{Polycentropis ierapetra MaLICKY, 1972}

Auf den Ionischen Inseln nur von Korfu und Kefalonia mit der subspec. dirfis MALICKy, 1974 bekannt, u.a. ein $1 o^{\top}$ vom Aviathos lake am 30.04.2003, leg WEIDLICH (Malicky, 2005: 83, 184). Neue Funde ebenfalls am Aviathos lake vom 04./05.05.2015 (1 1 und 3 ㅇ ㅇ , LF. und FL.). Auf Zakynthos und Kythira kommt die subspec. kalliope MALICKY, 1976 vor (MALICKY, 2005: 84, 184).

\section{Psychomyiidae}

\section{Tinodes braueri McLaCHLAN, 1878}

MaLICKy gibt nur wenige Funde von Korfu und Kefalonia an (Malicky, 2005: 88, 189). Nun ein o und 5 우 우 am Aviathos lake am 04./05.05.2015 (LF. und FL.).

\section{Hydropsychidae}

\section{Hydropsyche instabilis CURTIS, 1834}

Von den Ionischen Inseln nur von Kefalonia bekannt, u.a. $3 o^{\star} o^{\star}$ vom Aviathos lake am 30.04.2003, leg. Weidlich (Malicky, 2005: 97, 194). Neue Funde am selben Fundort: $14 \sigma^{\star} o^{\star}$ und 5 우 am 04./05.05.2015 (LF. und FL.).

\section{Limnephilidae}

\section{Micropterna testacea GMELIN, 1789}

Von den Ionischen Inseln nur von Kefalonia bekannt Nationalpark „Enos“, Osthang, 1977, leg. MALICKY und Aviathos lake, 2003, leg. Weidlich (Malicky (2005: 119, 219). Am 06.05.2015 flogen am frühen Morgen zwischen 6 und 8 Uhr (OESZ) auf dem EnosGipfel im Nationalpark etliche Exemplare zwischen Abertausenden Tipuliden.

\section{Stenophylax mitis McLaCHLAN, 1875}

MALICKY (2005: 122, 222) erwähnt ein einzelnes ơ von Lefkada (Eglouri) und wenige Funde von Kefalonia. Neuerdings der Zweitfund für Lefkada, ein Pärchen am 04.05.2015 bei Katohori in der Lichtfalle.

\section{Leptoceridae}

Triaenodes ochreellus McLACHLAN, 1875

Die Art kommt in ihrer subspec. lefkas nur auf den Ionischen Inseln, dem gegenüber liegenden Festland und der Peloponnes vor. Von Kefalonia nur wenige Funde von Kulurata, leg. MALICKY und Aviathos lake, leg. Weidlich (MAlicky, 2005: 127, 128, 231). Aktuell erschienen $2 \sigma^{*} \sigma^{*}$ am 04.05.2015 wiederum am Aviathos lake am Licht.

\section{Auswertung der faunistischen Angaben}

In der vorliegenden Arbeit werden die Daten zur Schmetterlingsfauna der Ionischen Inseln zusammengetragen. Im Ergebnis ergibt sich bei den Lepidopteren eine Gesamtartenzahl von 638. Weiterhin wird beschrieben, dass der lepidopterologische Kenntnisstand über die einzelnen Inseln sehr unterschiedlich ist.

Die historisch schon lange gut untersuchte festlandsnahe Insel Korfu weist den höchsten Artenbestand auf, der sich derzeit auf 432 beläuft. Die noch nähere an das Festland gelegene Insel Lefkada zeigt dagegen, abhängig vom geringeren Erforschungsgrad, mit 187 Arten eine deutlich geringere Artenzahl auf. Das festlandsfernere und kleine Ithaka verfügt über 188 Arten. Von der großen und naturräumlich verschieden ausgestatteten Insel Kefalonia sind 258 Arten bekannt geworden. Von Zakynthos liegen Daten von 117 und von Kythira 58 Schmetterlingsarten vor. Meganisi mit 8 Arten und Vido mit 2 und Paxos mit 3 sowie Sapientza und Elafinissos mit einer und Antikythira mit 4 Arten fallen dagegen deutlich ab und weisen somit auf besonders große Beobachtungslücken hin. Speziell bei den Tagfaltern ergibt sich heute folgender Wissensstand: Inselgruppe Ionische Inseln 99 Arten $(+1)$, Korfu 96 Arten $(+1)$, Lefkada $44(+2)$, Ithaki $45(+42)$, Kefalonia 61 (+2), Zakynthos 39, Kythira 43 Arten, Vido 2 Arten, Meganisi 3 Arten, Sapientza und Elafinissos mit jeweils einer und Antikythira mit 4 Arten.

Für die übrigen Gruppen ist eine Vergleich wenig aussagekräftig und es könnte lediglich die Famile Psychidae herausgehoben werden, die mit zwei aus der Literatur bekannten Arten sowie zwei incertae sedis, derzeit über 13 Arten auf den Inseln verfügt.

Es ist wahrscheinlich, dass sich die Gesamtartenzahl der Ionischen Inseln bei intensiver Erforschung noch um mehrere Hundert Arten erhöhen wird.

$\mathrm{Zu}$ den drei bisher bekannten endemischen Taxa der Ionischen Inseln, eine Art N. scholzi kommt ausserdem auch noch auf Kreta vor, werden eine neue Art Bankesia cephalonica spec. nov. und weitere drei neue Taxa von einer weiteren Psychidenart bekanntgegeben (Reisseronia ionica spec. nov.). Es ist anzunehmen, dass insbesondere auf dem Enos noch weitere endemische Arten vorkommen. 


\section{Danksagung}

Ich möchte mich bei meinen Freunden und Kollegen ausdrücklich bedanken, die beim Zustandekommen dieser Arbeit behilflich waren. Insbesondere gilt dies für W. R. Arnscheid (Bochum/Deutschland), der mich in der Diskussion und in der kritischen Durchsicht des Manuskriptes vielfältig unterstützte und für die Arbeit wichtige Genitalpräparate und -fotos anfertigte. Weiterhin danke ich H. BLACKsTeIn (Rathenow/ Deutschland) und Z. TokÁr (Michalovce/Slowakei) für Genitaldeterminationen, Dr. P. Huemer (Innsbruck/Österreich) für organisatorische Unterstützung für die DNA-Analysen in Canada, A. DAHL (Spörkelnbruch/Deutschland), G. EMBACHER (Salzburg/ Österreich) und Dr. C. PAPÉ (Lörrach/Deutschland) für die Bereitstellung weiterer faunistischer Daten, Prof. Dr. Z. LAšTŮvKA, (Brno/Tschechische Republik) für Hinweise zu den Sesiidae, V. Mironov (Moskau/ Rußland) für die Determination einiger Eupithecien und nicht zuletzt Dr. W. MEY (Berlin/Deutschland) für systematisch-taxonomische Hinweise und kritische Durchsicht des Trichopterenteils.

\section{Literatur}

Arenberger, E. 1983: Records of the Lepidoptera of Greece based on the collections of G. Christensen and L. Gozmány: II, Pterophoridae. - Annales Musei Goulandris 6: 199-206.

Arnscheid, W. R. \& Weidlich, M. 2016: Psychidae. In: Karsholt, O.; Mutanen, M. \& Nuss, M. (eds), Microlepidoptera of Europe, Volume 8: Brill, Leiden (in print).

BAldizzone, G. 1983: Records of the Lepidoptera of Greece based on the collections of G. Christensen and L. Gozmány: III, Coleophoridae. - Annales Musei Goulandris 6: 207-248.

BAldock, D. W. \& Bretherton, R. F. 1981: Butterflies in Corfu (Kerkyra) in late August, 1980. Part II a provisional list of the butterflies of Corfu (Kerkyra). - Proceedings and Transactions of the British Entomological and Natural History Society $14(3 / 4)$ : 8-10, 101-107.

Buresch, I. \& Iltschew, D. 1915: Zweiter Beitrag zur Erforschung der Lepidopterenfauna von Thrazien, Mazedonien und Nachbarländern. - Arbeiten der Bulgarischen Naturforschenden Gesellschaft 8: 151-197.

De La Garde, P. 1899: Mediterranean Lepidoptera. Entomologist: 8-11.

de WaArd, J. R.; Ivanova, N. V.; Hajibabaei, M. \& Hebert, P. D. N. 2008: Assembling DNA Barcodes. Analytical Protocols: 275-293. - In: Cristofre, M. (Hg.): Methods in Molecular Biology: Environmental Genetics. Humana Press Inc., Totowa, USA: 364 pp.
EmbacheR, G. 2000: Kleiner Beitrag zur Lepidopterenfauna Griechenlands (Insecta: Lepidoptera). - Zeitschrift Arbeitsgemeinschaft Österreichischer Entomologen 52: 65-70.

ERBER, J. 1866: Ergebnisse der diessjährigen Reise nach Griechenland. - Verhandlungen der zoologisch-botanischen Gesellschaft in Wien 16: 825-828.

ErbeR, J. 1867: Bemerkungen zu meiner Reise zu den griechischen Inseln. - Verhandlungen der zoologisch-botanischen Gesellschaft in Wien 17: 853-856.

Fibiger, M.; Ronkay, L.; Yela, J. L. \& Zilli, A. 2010: Noctuidae Europaea Volume 12 Rivulinae-Euteliinae, and Micronoctuidae and Supplement to Volume 1-11: 1-451, Entomological Press, Sorø.

Fibiger, M.; László, G. M.; Ronkay, G.; Ronkay, L.; Speidel, W.; Varga, Z.; Wahlberg, N.; Witt, T. J.; Yela, J. L.; Zahiri, R. \& Zilli, A. 2011: Noctuidae Europaeae Volume 13 Lymantriinae and Arctiinae including Phylogeny and checklist of the Quadrifid Noctuidea of Europe: 1-448, Entomological Press, Sorø.

Freina, J. J. DE 1999: Die Bombyces und Sphinges der Westpaläarktis. Band IV Sesiidae: 1-432, Edition Forschung \& Wissenschaft Verlag $\mathrm{GmbH}$, München.

FreinA, J. J. DE \& Piatkowski, H.-J. 2006: Beitrag zur Erfassung der Heteroceren Griechenlands (Lepidoptera). - Entomologische Zeitschrift 116 (6): 243-260.

Freina, J. J. DE \& WitT, T. 2001: Die Bombyces und Sphinges der Westpaläarktis. Band III Zygaenidae: 1-575, Edition Forschung \& Wissenschaft Verlag $\mathrm{GmbH}$, München.

Galvagni, E. 1934/1935: Griechische Falter, insbesondere über die auf der Griechenlandreise der Universität Wien, Ostern 1933, 8.-23. April beobachteten Schmetterlinge. - Zeitschrift des Österreichischen Entomologischen Vereines 19: 74-76, 20 : 5-7.

Gozmany, L. 1983: Records of the Lepidoptera of Greece based on the collections of G. Christensen and L. Gozmány: IV, Diverse families of Microlepidoptera. - Annales Musei Goulandris 6: 253-262.

Gozmány, L. 1985: Records of the Lepidoptera of Greece based on the collections of G. Christensen and L. Gozmány: XII, Phycitidae. - Annales Musei Goulandris 7: 369-373.

Gozmány, L. \& Acs, E. 1990: Records of the Lepidoptera of Greece based on the collections of G. Christensen and L. Gozmány: XVI, Autumnal Microlepidoptera. Annales Musei Goulandris 8: 255-262.

Hacker, H. H. 1989: Die Noctuidae Griechenlands. Mit einer Übersicht über die Fauna des Balkanraumes (Lepidoptera, Noctuidae). - Herbipoliana 2: 1-589.

Hacker, H. H. 1996a: Die Noctuidae Griechenlands. Mit einer Übersicht über die Fauna des Balkanraumes (Lepidoptera, Noctuidae) 2. Nachtrag. - Esperiana 4: 245-261. 


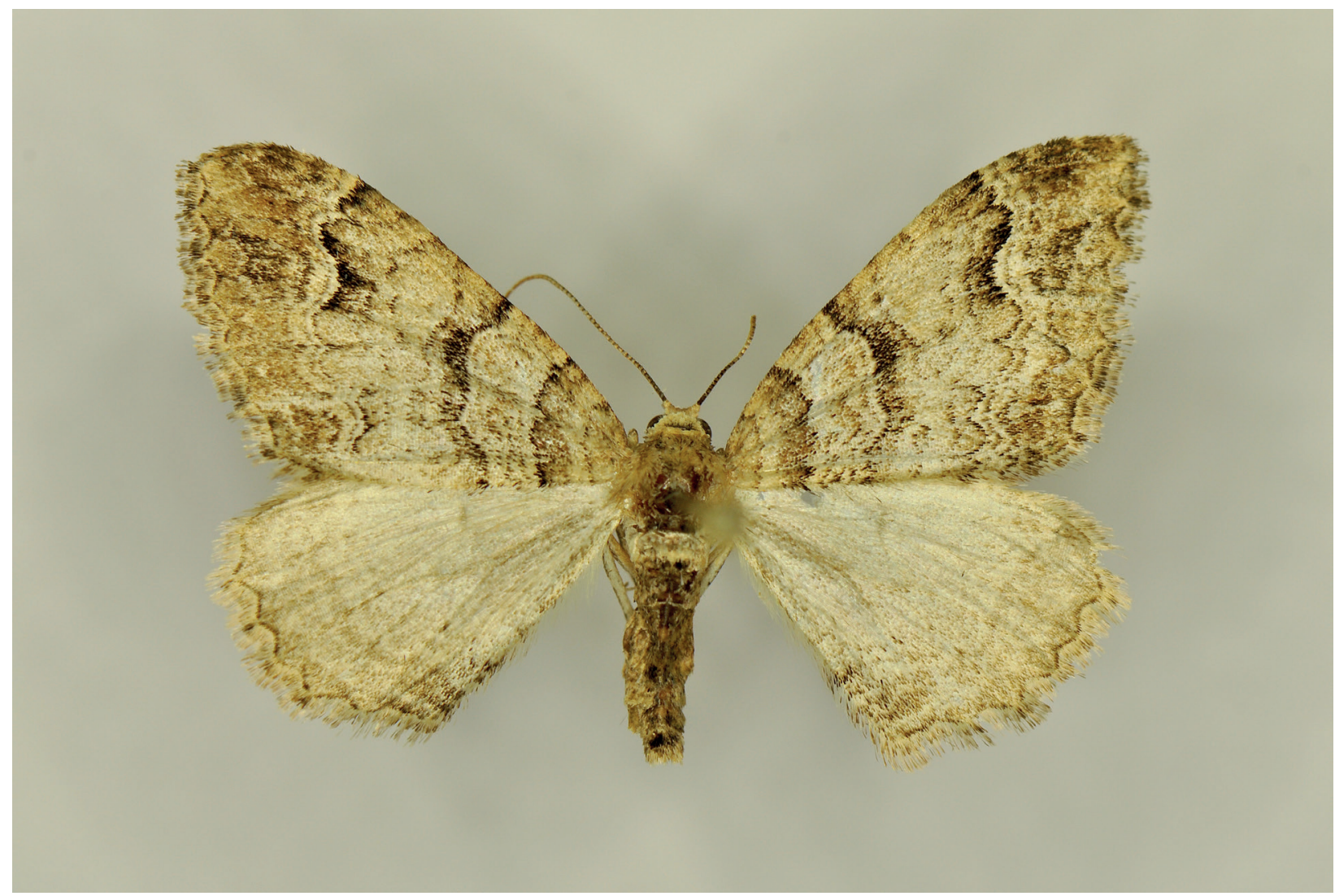

Fig. 10: Catorrhoe hortulanaria (STAUdINGER, 1879), Ithaka, Vathi, 1 †, 05.05 .2003 (LF.) (Flügelspanne: 21 mm, Foto: M. WEIDLICH).

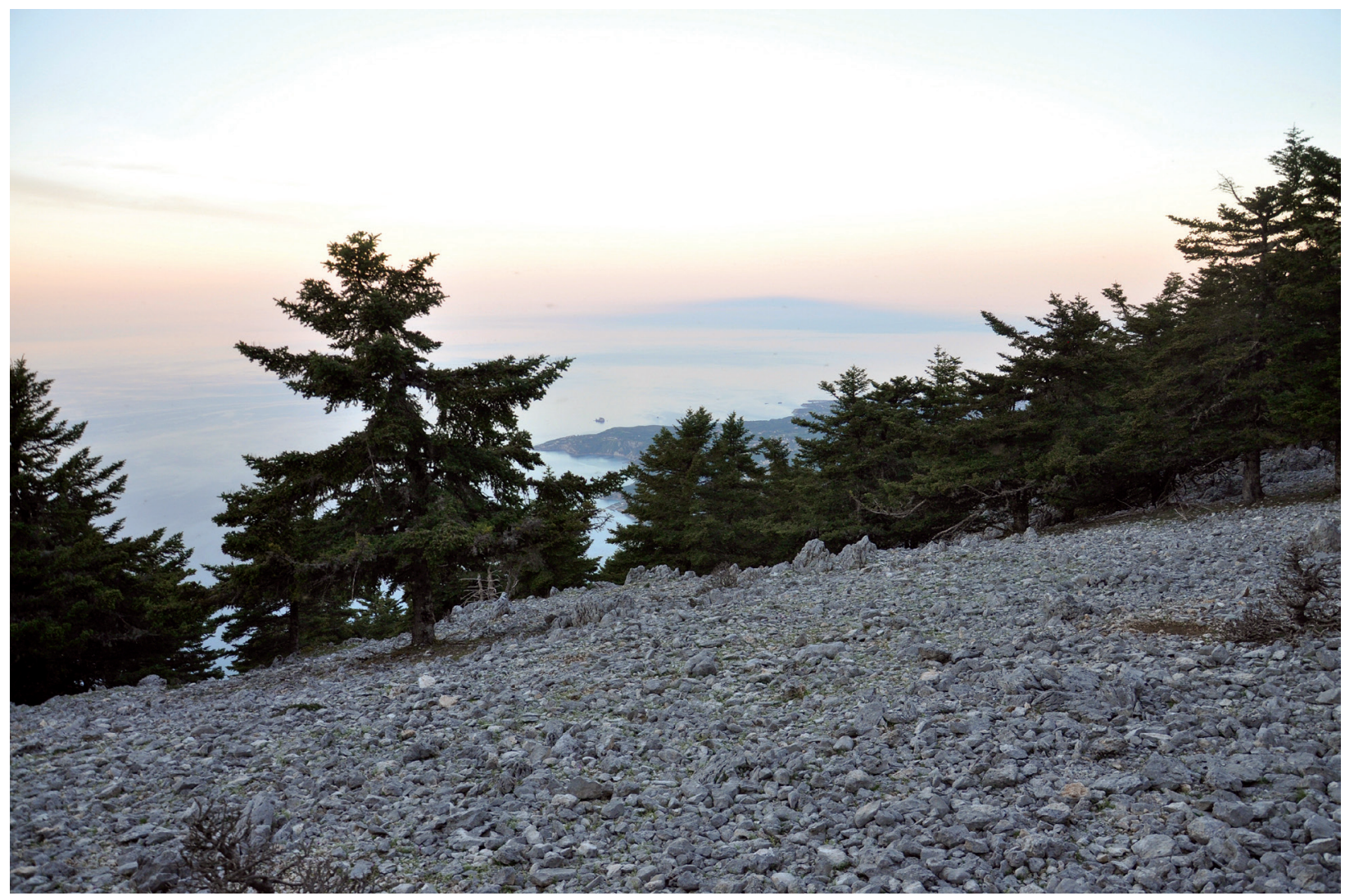

Fig. 12: Lebensraum von Bankesia cephalonica spec. nov., Kefalonia, Nationalpark Enos-Gipfel, 1600 m NN (Foto: M. WeIDLICH, 05.05.2015) 


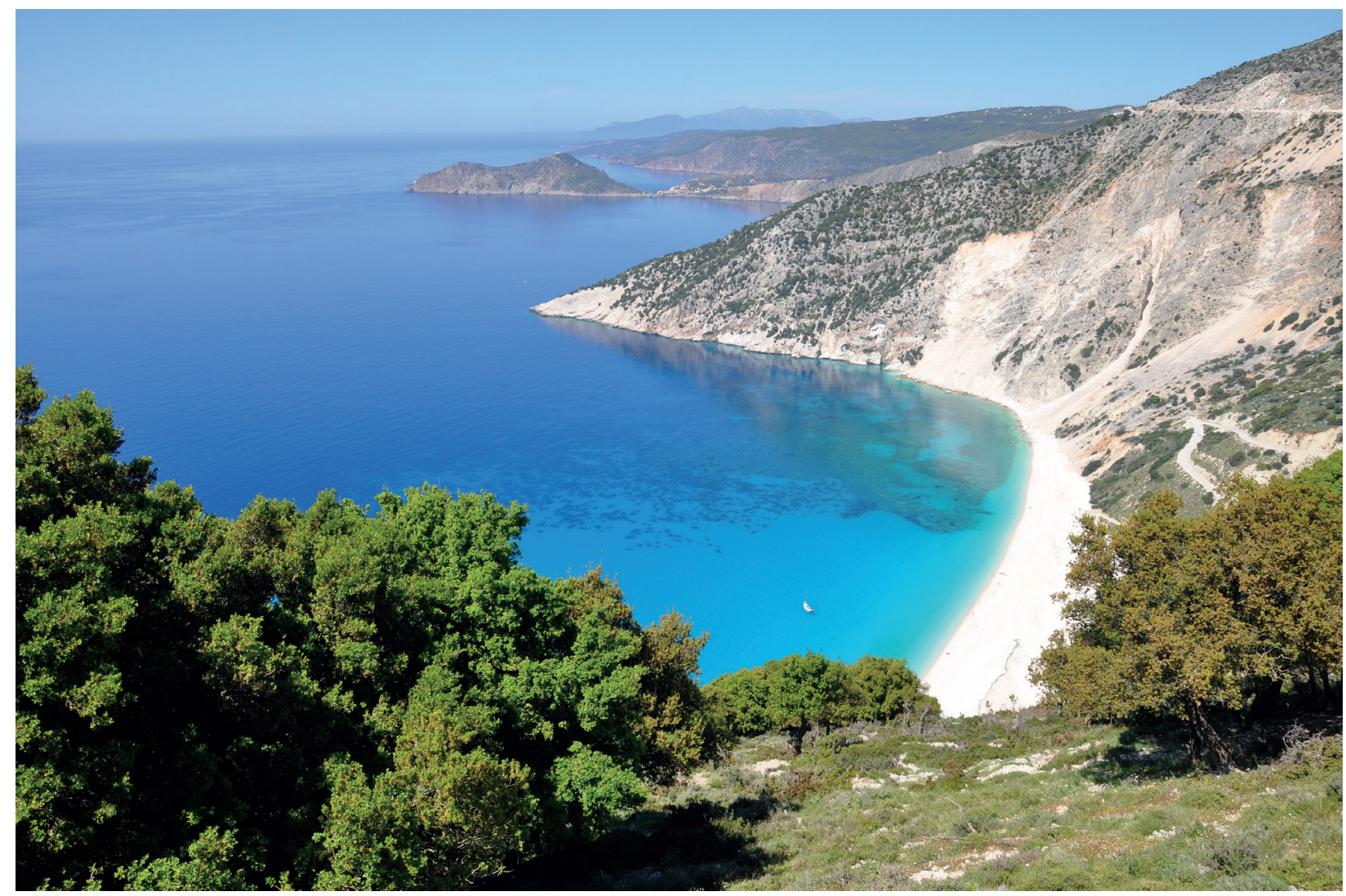

Fig. 13: Lebensraum von Reisseronia ionica spec. nov., Kefalonia, Westhänge des Skoluopas und Myrto Bay (Foto: M. WEIDLICH, 04.05.2015).

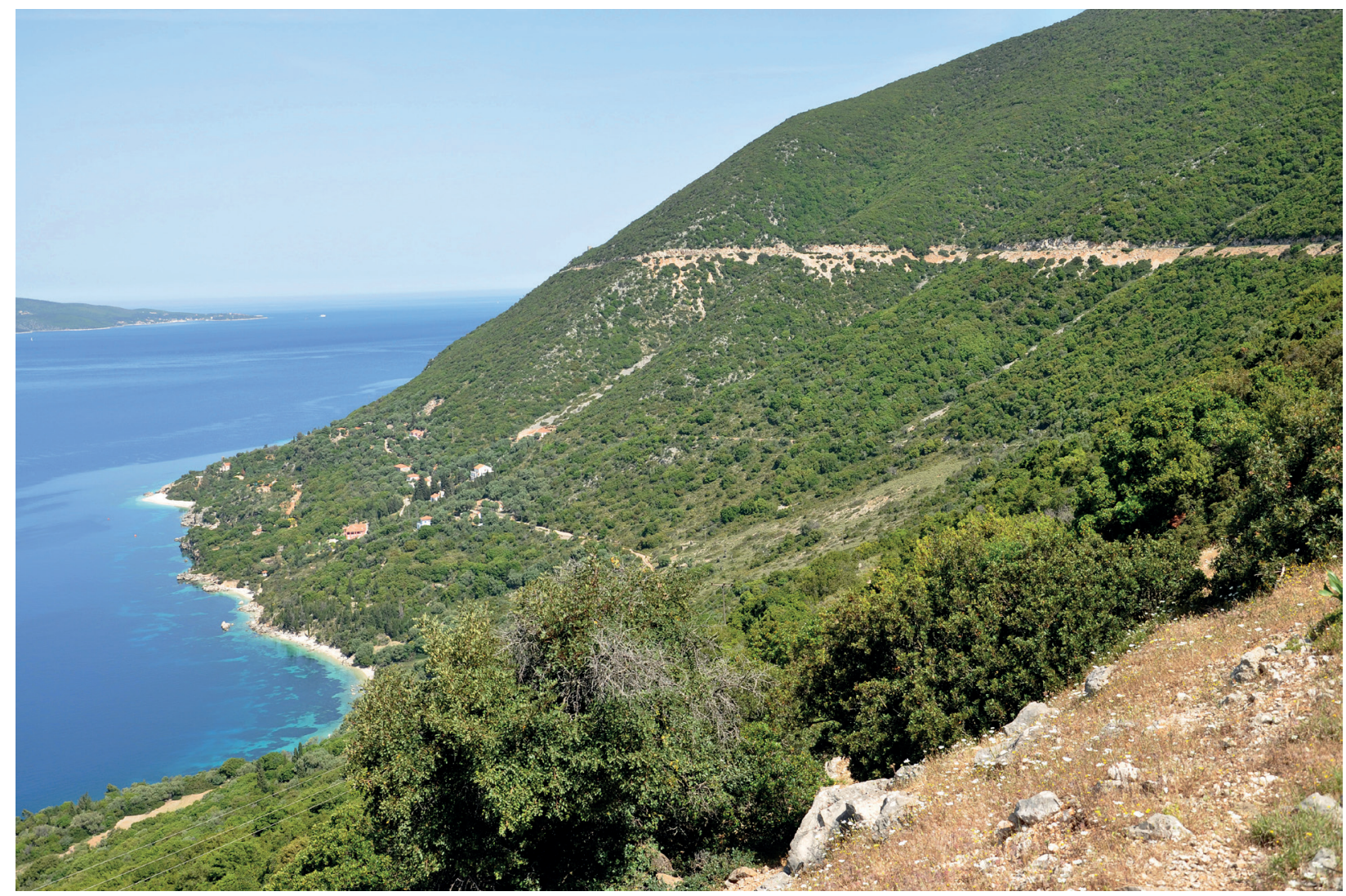

Fig. 14: Lebensraum von Reisseronia ionica odysseus subspec. nov., Ithaka, Umg. Lefki (Foto: M. WeIDLICH, 06.05.2015). 
Hacker, H. H. 1996b: Revision der Gattung Hadena Schrank, 1802 (Lepidoptera: Noctuidae). - Esperiana 5: 1-696.

Hättenschwiler, P. 1982: Eine neue Reisseronia aus dem Peloponnes (Psychidae). - Nota lepidopterologica 5 (1): 25-29.

Hättenschwiler, P. 1990: Melasina christenseni sp. n. (Lepidoptera, Psychidae), eine neue Psychide aus dem südlichen Peloponnes. - Nota lepidopterologica 12 (4): 257-261.

Hauser, E. 2012: Revision der Gattung Rebelia Heylaerts 1900 (Lepidoptera, Psychidae. - Linzer biologische Beiträge 44 (1): 181-306.

Hausmann, A. 2001: The Geometrid Moths of Europe. Volume 1. Archiearinae, Orthostixinae, Desmobathrinae, Alsophilinae, Geometrinae: 1-282, Apollo Books, Stenstrup.

Hausmann, A. 2004: The Geometrid Moths of Europe. Volume 2. Sterrhinae: 1-600, Apollo Books, Stenstrup.

Hausmann, A. 2011: An integrative taxonomic approach to resolving some difficult questions in the Larentiinae of the Mediterranean region (Lepidoptera, Geometridae). - Mitteilungen der Münchner Entomologischen Gesellschaft 101: 73-97.

Hausmann, A. \& Viidalepp, J. 2012: The Geometrid Moths of Europe. Volume 3. Subfamily Larentiinae I: 1-743, Apollo Books, Stenstrup.

Hofmann, A. 2003: Zygaena (Zygaena) ephialtes (Linnaeus, 1767) im südlichen Balkan nebst Anmerkungen zur Entstehung von Polymorphismus sowie melanistischer Zygaena-Formen im Mittelmeerraum (Lepidoptera: Zygaenidae). - Entomologische Zeitschrift 113: Teil 1: (2): 50-54, Teil 2: (3): 75-86, Teil 3: (4): 108-119.

HolıK, O. 1939: Beiträge zur Kenntnis der Zygaenen Südosteuropas. (Fortsetzung). - Mitteilungen der Münchner Entomologischen Gesellschaft $29(2 / 3)$ : 173-206.

Hynd, W. R. B. 1981: Notes and Observations. Records of Zygaenidae (Lepidoptera) from Corfu, Greece. Entomologist's Gazette 32: 283.

Karsholt, O. \& Razowski, J. (eds.) 1996: The Lepidoptera of Europe: 380 pp., Apollo Books, Stenstrup.

Kattioulas, M. \& Koutsafiktis, A. 1977: Lepidopterenfauna der Insel Kythera, I. Teil. - Annales Musei Goulandris 3: 115-120.

KNAPp, R. 1965: Die Vegetation von Kephallinia, Griechenland: 1-206, Königstein.

KöstLeR, W. 1991: Entomologische Reiseeindrücke von der Insel Korfu (Griechenland). - Galathea 7 (3): 100-104.

Landry, J.-F.; Nazari, V.; DewaArd, J. R; MutaNen, M.; Lopez-VAamonde, Huemer, P. \& Hebert, P. D. N. 2013: Shared but overlook: 30 species of Holarctic Microlepidoptera revealed by DNA barcodes and morphology. - Zootaxa 3749 (1): 1-93.
LAŠTU゚VKa, Z.; MALICKy, H.; HÜtTinger, E.; Rausch, H. \& Ressl, F. 1990: Sesien-Funde aus Europa und dem Mediterrangebiet (Lepidoptera, Sesiidae). Zeitschrift Arbeitsgemeinschaft Österreichischer Entomologen 41 (3/4): 105-110 (1989).

LUNAK, R. 1941: Die Lebensweise von Ocnogyna parasita Нв. und ihre Biotope. - Zeitschrift des Wiener Entomologischen Vereins 26 (3): 65-70.

Malicky, H. 1992: Faunistische Meldungen von Lepidopteren aus Griechenland und Zypern. Esperiana 3: 391-407.

Malicky, H. 2005: Die Köcherfliegen Griechenlands. Denisia 17: 1-240, Linz.

Mathew, G. F. 1898: Notes on Lepidoptera from the Mediterranean. - Entomologist: 108-116.

Meier, H. G. 1963: Zur Kenntnis der Gattungen Pseudobankesia gen.nov. und Bankesia TutT (Lep., Psychidae). - Mitteilungen der Münchner Entomologischen Gesellschaft 53: 1-23.

Mironov, V. 2003: The Geometrids Moth of Europe. Volume 4, Larenthiinae II (Perizomini and Eupitheciini): 1-463, Apollo Books, Stenstrup.

NeL, J. 1999: Espèces nouvelles ou rarement signalées de microlépidoptères de France. - Bulletin de la Société entomologique de France 104 (4): 347-355.

Norris, F. B. 1891: Notes on Rhopalocera in Corfu. Entomologist: 179-180.

PAMperis, L. N. 2009: The Butterflies of Greece: 1-766, Athens.

Papapavlou, K. \& Katsouni, N. 2008: New records and additional biogeographical data on the Lepidoptera (Papilionoidea) and Orthoptera fauna of the islands of Cephalonia and Ithaki, Ionian Sea, Greece. Entomologist' Gazette 59: 27-39.

PARker, R. 2010: Cacyreus marshalli Butler, 1898 (Lepidoptera, Lycaenidae) newly recorded for Corfu, with notes on other butterflies on the Island in September 2008. - Entomologist's Gazette 61: 40-42.

Petersen, G. \& Gaedike, R. 1983: Records of the Lepidoptera of Greece based on the collections of G. Christensen and L. Gozmány: VII, Tineidae, Epermeniidae, Acrolepiidae, Douglasiidae. - Annales Musei Goulandris 6: 271-311.

Petersen, G. \& Gaedike, R. 1990: Nachtrag zur Lepidopterenfauna Griechenlands (Tineidae, Epermeniidae, Acrolepiidae, Douglasiidae). - Annales Musei Goulandris 8: 291-307.

Piatkowski, H.-J. 2006: Beitrag zur Erfassung der Noctuidae Griechenlands (Insecta: Lepidoptera). Atalanta 37 (3/4): 345-392, 495-507.

Predovnik, Ž. 2003: Clearwing Moth (Lepidoptera: Sesidae) in the central Lepidoptera collection of the Slovenian Museum of Natural History. - Acta entomologica Slovenica 11 (2): 171-182.

Ratnasingham, S. \&Hebert, P.D. N.2013: A DNA-Based Registry for All Animal Species: The Barcode Index Number (BIN) System. PLOS ONE 8 (8): e66213. 
Rebel, H. 1901: Catalog der Lepidopteren des palaearctischen Faunengebietes. II. Theil: Famil. Pyralidae-Micropterygidae: 1-368, Berlin, R. Friedlander \& Sohn.

Rebel, H. 1910: Beitrag zur Lepidopterenfauna der Ionischen Inseln. - Verhandlungen der zoologischbotanischen Gesellschaft in Wien 50: 418-431.

Rebel, H. 1912: Nachtrag zur Lepidopterenfauna von Korfu. - Verhandlungen der zoologisch-botanischen Gesellschaft in Wien 52: (12)-(15).

ReBel, H. 1915: Beitrag zur Lepidopterenfauna Griechenlands. - In: Bericht der Sektion für Lepidopterologie. Versammlung am 4. Dezember 1914. - Verhandlungen der zoologisch-botanischen Gesellschaft in Wien 65: (50)-(59).

ReBEL, H. 1924: Lepidopterologische Nachträge zu einigen ostmediterranen Insularfaunen. - Jahresberichte Wiener entomologischer Verein 30: 37-49.

Rebel, H. 1932: Griechische Lepidopteren. - Zeitschrift des Österreichischen Entomologen-Vereines 17 (8): 53-56.

Rebel, H. 1933: Griechische Lepidopteren II. - Zeitschrift des Österreichischen Entomologen-Vereines 18: 91.

Rebel, H. 1937: Griechische Lepidopteren V. - Zeitschrift des Österreichischen Entomologen-Vereines 22 (10): 93-95.

Rebel, H. 1938: Mitteilungen über Canephorinen und über Solenobia triglavensis RBL. - Zeitschrift des Österreichischen Entomologen-Vereines 23 (8): 77-80.

Skou, P. \& Sinvonen, P. 2015: Subfamily Ennominae I. - In: Hausmann, A. (ed.): The Geometrid Moths of Europe. Volume 5: 1-657, Brill, Leiden.

Speidel, W. 1984: Revision der Acentropinae des palaearktischen Faunengebietes (Lepidoptera: Crambidae). - Neue Entomologische Nachrichten 12: $1-157$.

ŠpatenKa, K. 1997: Neue Glasflügler-Arten und Unterarten aus Europa und der Türkei (Sesiidae, Lepidoptera). - Bonner zoologische Beiträge 47 (1-2): 43-57.

ŠpatenkA, K. \& LAštůvkA, Z. 1990: Zur Taxonomie von Bembecia scopigera (Scopoli, 1763), B. ichneumoniformis ([DENIS \& SCHIFFERMÜLLER], 1775) und B. albanensis (Rebel, 1918) (Lepidoptera, Sesiidae). Entomofauna 11 (5): 109-121.

StAudinger, O. 1871: Beitrag zur Lepidopterenfauna Griechenlands. - Horae Societatis Entomologicae Rossicae 7: 1-304.

StAudinger, O. 1879: Lepidopteren-Fauna Kleinasien's. - Horae Societatis Entomologicae Rossicae 15: 159-368, pars.

Stengel D. 1990: Drei neue Psychidae-Arten aus Griechenland und ein neuer Status für Peleponnesia glaphyrella culminella Sieder (Lepidoptera, Psychidae). - Atalanta 20: 211-217.
Sutter, R. 2000: Nemapogon scholzi sp.n. aus Griechenland (Insecta: Lepidoptera: Tineidae). Reichenbachia, Staatliches Museum für Tierkunde Dresden 33 (56): 427-428.

Tolmann, T. \& Bernhard, T. 1994: Significant extensions to the known range of Anthocharis damone Boisduval, 1836 in Greece (Lepidoptera: Pieridae). Phegea 22 (4): 177-180.

Walsingham, L. 1919: New species of Aristotelia and Micropteryx. - The Entomologist's Record and Journal of Variation 31 (1): 10-12.

WeIDlich, M. 1989: Abriß der Psychidenfauna Bulgarisch-Mazedoniens mit der Erstbeschreibung des Weibchens und Sackes von Reisseronia nigrociliella (ReBel, 1934) (Lepidoptera, Psychidae). - Nachrichtenblatt der Bayerischen Entomologen 38 (1): 1-12.

WeIDlich, M. 2006: Reisseronia arnscheidi sp. n. aus den Südkarpaten Rumäniens (Psychidae). - Nota lepidopterologica 28 (3/4): 203-211.

Weidlich, M. 2008: Beitrag zur Lepidopteren-Fauna des Notia Pindos (Tringia Massiv, Lákos-Gebirge und Athamáno-Gebirge in Griechenland mit Beschreibung von zwei neuen Arten sowie Angaben zur Köcherfliegen- (Trichoptera) und Schnakenfauna (Diptera: Tipulidae). - Entomofauna 29 (27): 469-504.

Weidlich, M. 2012a: Zur Kenntnis von Loebelia crassicornis (STAUDINGER, 1871) (Lepidoptera: Psychidae). - Entomologische Zeitschrift $122(2)$ : 79-85.

WeidLICH, M. 2012b: Zur Schmetterlings-Fauna der Zagoria im Voria Pindos (Griechenland) mit der Beschreibung einer neuen Psychidenart (Lepidoptera, Psychidae). - Linzer biologische Beiträge $44(2)$ : 1237-1262.

Weidlich, M. 2013: Zur Psychidenfauna Albaniens unter Berücksichtigung der Albanien-Expedition 1961 des Deutschen Entomologischen Institutes. - Beiträge zur Entomologie 63 (2): 324-338.

WeIDLICH, M. 2016: Pseudobankesia lichenaria spec. nov., eine neue Psychiden-Art aus der Zagoria im griechischen Voria Pindos-Gebirge (Lepidoptera: Psychidae). - Beiträge zur Entomologie $66(1)$ : 131-138.

WitT, T. 1980: Die Verbreitung und Rassenbildung von Ocnogyna parasita (Hübner, 1790) (Lepidoptera, Arctiidae). - Mitteilungen der Münchner Entomologischen Gesellschaft 69: 133-165. 


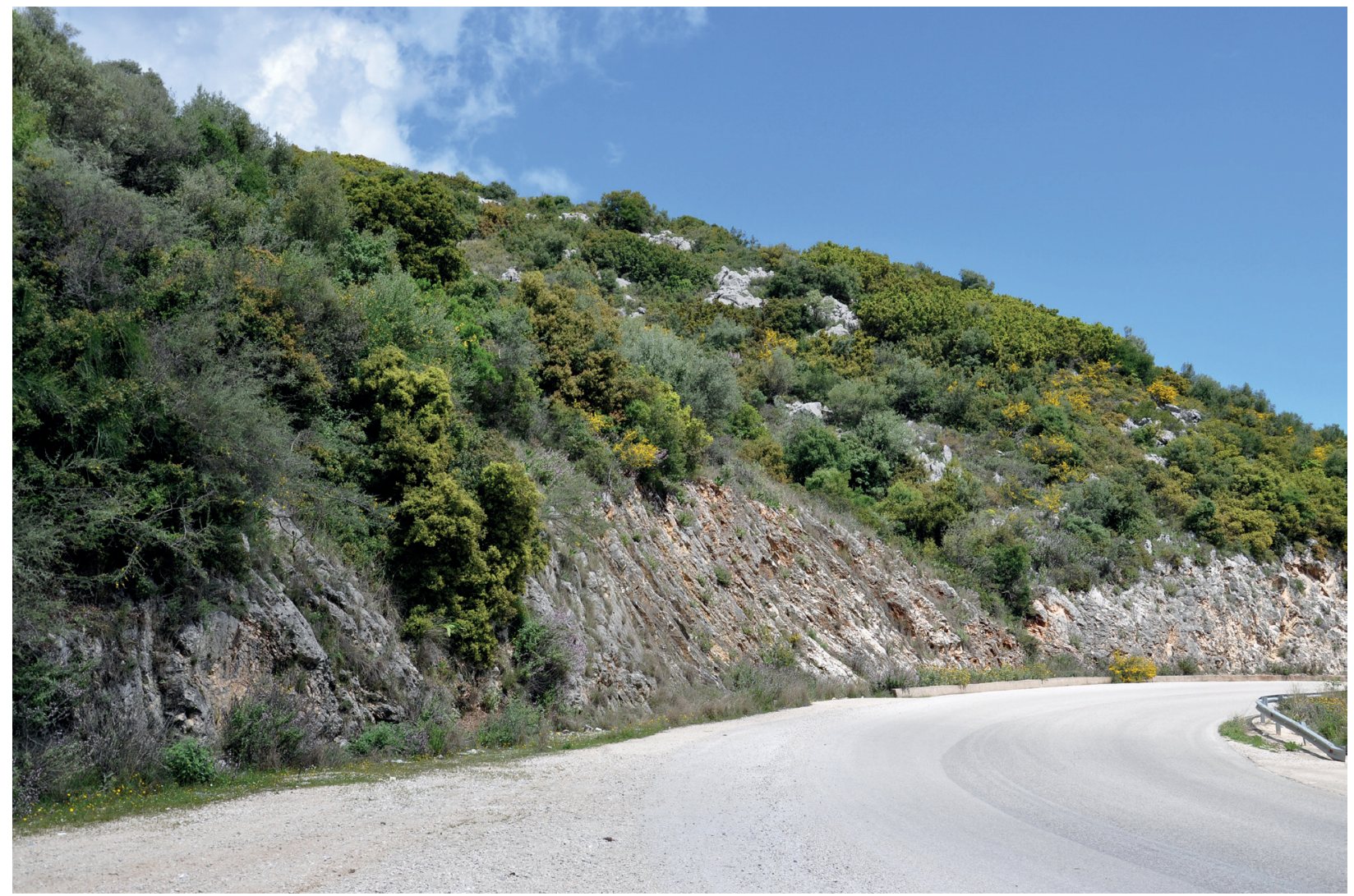

Fig. 15: Lebensraum von Reisseronia ionica lefkadensis subspec. nov., Lefkada, Umg. Vliko, 70 m NN (Foto: M. WEIDLICH, 21.04.2012).

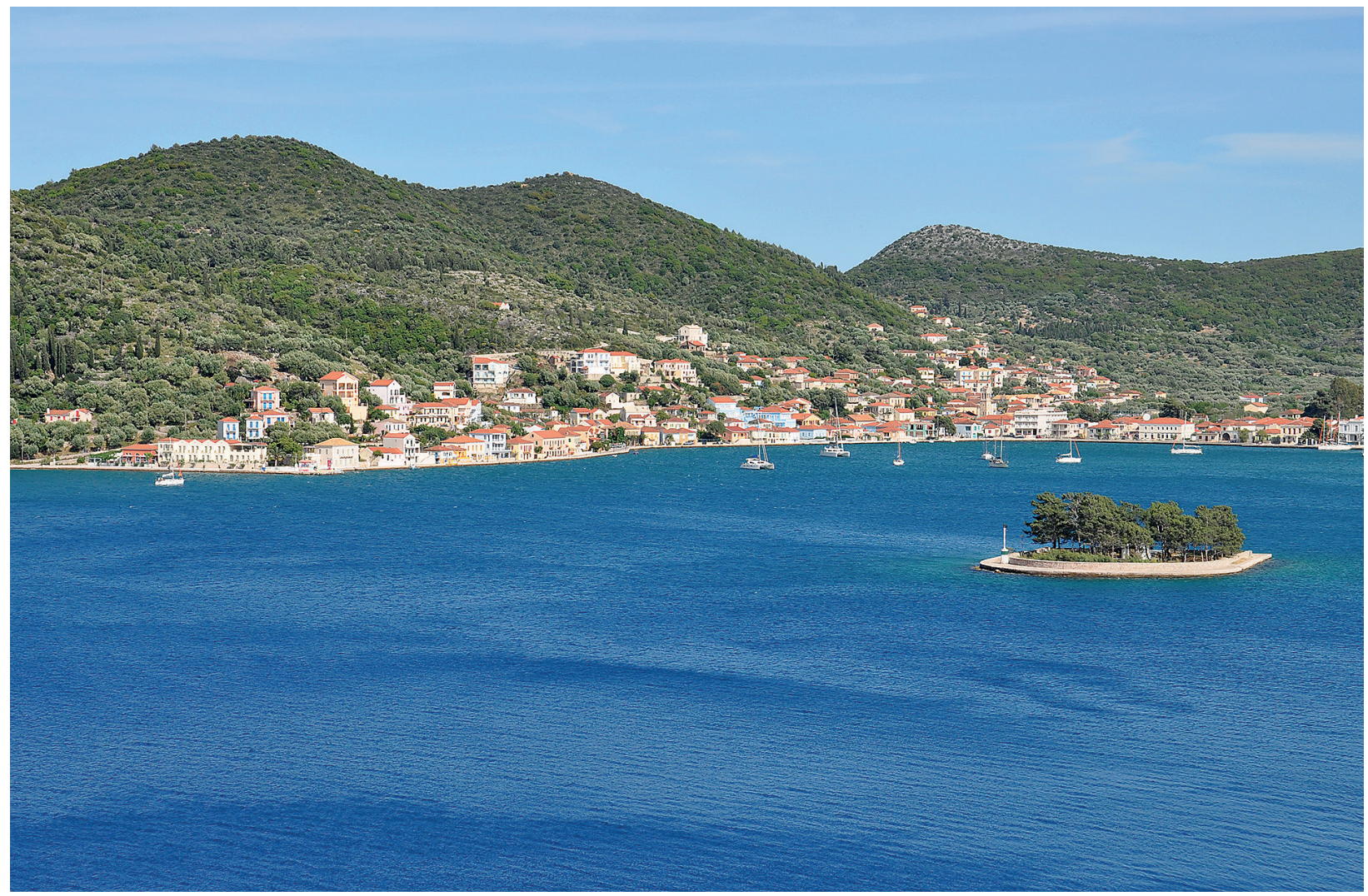

Fig. 16: Vathi auf Ithaka. Hier wurden u.a. Reisseronia ionica odysseus subspec. nov., Penestoglosa dardoinella Mill., Peleponnesia glaphyrella Rebel, Lobesia botrana Den. \& Sch., Scopula vigilata WAgner, Idaea albitorquata Püngeler, Oulobophora internata PÜNGeler, Zebeera falsalis H.-S., Caradrina wullschlegeli PÜNgeler, Hadena adriana SCHAwerda und Eilema costalis ZelleR gefunden (Foto: M. WeIDLICH, 04.05.2015). 
Tab. 1: Darstellung der Fundnachweise auf den Ionischen Inseln. Im Text werden zu den mit * markierten Arten Einzelheiten gegeben bzw. diskutiert. Die Abkürzungen bedeuten im Einzelnen:

AR = ARENBERGER (1983)

BAL $=$ BALDIZZONE (1983)

BLA = BLACKSTEIN (in litt. 2016)

BUR \& ILT = BURESCH \& ILTSCHEW (1915)

DAHL $=$ DAHL 2007 in www.lepiforum.de abgerufen am 06. und 13.09.2016

DE LA GARDE = DE LA GARDE (1899)

$\mathrm{EMB}=$ EMBACHER $(2000=$ ohne Tagfalter und Noctuidae, in litt. 2016)

$\mathrm{FR}=$ De FreINA (1999)

FR \& PI = De Freina \& PiATKowsKi (2006)

FRIE $=$ M. FRIEDRICH 2011 in www.lepiforum.de abgerufen am 06.09.2016

GAL $=$ GALVAGNI $(1934 / 1935)$

GINZ = GINZINGER 2013 in www.lepiforum.de abgerufen am 06.09.2016

GOZ = GOZMÁNY $(1983,1985)$

GOZ \& ACS = GOZMÁNY \& ACs (1990)

GRAF = GRAF 2014 in www.lepiforum.de abgerufen am 26.09.2016

HA = HACKER $(1989,1996 a, b)$

HAU $=$ HAUSMANN $(2001,2004)$

HAU \& VIID= HAUSMANN \& VIIDALEPP (2012)

HO $=$ HoLIK (1939)

HOF $=$ HOFMANN (2003)

$\mathrm{HU}=\mathrm{HUND} 2002$ in www.lepiforum.de abgerufen am 26.09.2016

HY $=$ HYND (1981)

KA \& KO = KatTIOULAS \& KoutsafiKTIS (1977)

KLEW = KLEWINGHAUS 2010 in www.lepiforum.de abgerufen am 06.09.2016

KÖ = KöSTLER (1991)

KRU = KRUMM 2008 in www.lepiforum.de abgerufen am 13.09.2016
LA = Laštůvka, Malicky, Hüttinger, Rausch \& Ressl (1990)

LANDRY = Landry, Nazari, DewaArd, Mutanen, LopezVaAmonde, Huemer \& Hebert (2013)

LU = LUNAK $(1941)$

MA = MALICKY $(1992,2005)$

MAT = MATHEW (1898)

MIR = MIRONOV (2003)

PA = PAMPERIS (2009)

PAP\&KA = PAPAPAVLOU \& KATSOUNI (2008)

PAPE $=$ PAPÉ 2012 in www.lepiforum.de abgerufen am 13.09.2016

PARK $=$ PARKER $(2010)$

PET \& GAE $=$ Petersen \& GAEDIKe $(1983,1990)$

PRE $=$ PREDOVNIK (2003)

PÜH = PÜHRINGER (LAŠTŮVKA in litt. 2016)

RE = RebeL $(1901,1910,1912,1915,1924,1932,1933,1937$, 1938)

SK \& SI = SKou \& SIHVONEN (2015)

$\mathrm{SP}=$ ŠPATENKA $(1997)$

SP \& LA = ŠPATENKA \& LAŠTU゚VKA (1990)

SPEI $=$ SPEIDEL $(1984)$

STAU $=$ STAUDINGER $(1871)$

STR $=$ STRIEKMANN 2007 in www.lepiforum.de abgerufen am 26.09.2016

STRU = STRUTZBERG 2008 in www.lepiforum.de abgerufen am 13.09.2016

SUT $=$ SUTTER $(2000)$

$\mathrm{VO}=$ VoNTZ 2011 in www.lepiforum.de abgerufen am 06.09 .2016

WAL $=$ WALSINGHAM (1919)

WE $=$ WeIDLICH Aufsammlungen (1993-2015)

$\mathrm{WI}=\mathrm{WITT}(1980)$

\begin{tabular}{|c|c|c|c|c|c|c|}
\hline Artenliste & Korfu & Lefkada & Ithaka & Kefalonia & Zakynthos & $\begin{array}{c}\text { Kythira/ } \\
\text { Kleine Inseln }\end{array}$ \\
\hline \multicolumn{7}{|l|}{ Lepidoptera } \\
\hline \multicolumn{7}{|l|}{ Micropterigidae } \\
\hline Micropterix aruncella Scopoli & $\mathrm{RE}$ & & & & & \\
\hline Micropterix corcyrella WALSINGHAM & WAL & & & & & \\
\hline \multicolumn{7}{|l|}{ Hepialidae } \\
\hline Triodia adriaticus OsTHELDER * & DAHL & & & & & \\
\hline Korscheltellus amasinus H. - S. & $\begin{array}{l}\text { STAU, } \\
\text { GOZ \& } \\
\text { ACS }\end{array}$ & & & & & \\
\hline \multicolumn{7}{|l|}{ Adelidae } \\
\hline Nemophora raddaella Нв. & STAU & & & & & \\
\hline Adela paludicolella ZELLER & & & & $\mathrm{RE}$ & & \\
\hline Cauchas rufifrontella TREITSCHKE & $\mathrm{RE}$ & & & & & \\
\hline \multicolumn{7}{|l|}{ Tineidae } \\
\hline Crassicornella crassicornella ZELLER & $\begin{array}{c}\text { PET \& } \\
\text { GAE }\end{array}$ & & & $\begin{array}{l}\text { RE, PET \& } \\
\text { GAE }\end{array}$ & & \\
\hline Cephimallota angusticostella ZELLER & $\begin{array}{c}\text { PET \& } \\
\text { GAE }\end{array}$ & & & & & \\
\hline Nemapogon scholzi SUTTER * & & & & & SUT & \\
\hline
\end{tabular}




\begin{tabular}{|c|c|c|c|c|c|c|}
\hline Artenliste & Korfu & Lefkada & Ithaka & Kefalonia & Zakynthos & $\begin{array}{c}\text { Kythira/ } \\
\text { Kleine Inseln }\end{array}$ \\
\hline Niditinea fuscella L. & $\begin{array}{l}\text { RE, PET \& } \\
\text { GAE }\end{array}$ & & & & & \\
\hline Monopis obviella D. \& S. & $\begin{array}{c}\text { PET \& } \\
\text { GAE }\end{array}$ & & & & & \\
\hline Hapsifera luridella ZELLER & $\begin{array}{l}\text { RE, PET \& } \\
\text { GAE }\end{array}$ & & & & & \\
\hline \multicolumn{7}{|l|}{ Psychidae } \\
\hline Eumasia parietariella HEYDENR. * & & WE & WE & $\begin{array}{l}\text { RE, GOZ, } \\
\text { WE }\end{array}$ & & \\
\hline Bankesia cephalonica spec. nov. ${ }^{*}$ & & & & WE & & \\
\hline Typhonia spec.* ${ }^{*}$ & & & & WE & & \\
\hline Penestoglossa dardoinella MiLl.* & & & WE & WE & & \\
\hline Luffia lapidella GoEzE * & & & WE & RE, WE & & \\
\hline Psyche crassiorella BRUAND * & & WE & & & & \\
\hline Peleponnesia glaphyrella ReBEL * & & RE, WE & WE & WE & & \\
\hline Reisseronia ionica spec. nov. ${ }^{*}$ & & WE & WE & WE & & \\
\hline Heliopsychidea graecella MiLL. ${ }^{*}$ & & & WE & WE & & \\
\hline Canephora hirsuta PoDA * & & & WE & WE & & \\
\hline Pachythelia villosella OCHSENHEIMER * & & WE & WE & WE & & \\
\hline Oiketicoides lutea STAUDINGER * & & & WE & $\mathrm{RE}$ & & \\
\hline Apterona helicoidella VALLOT * & & WE & WE & WE & & \\
\hline \multicolumn{7}{|l|}{ Gracillariidae } \\
\hline Phyllonorycter lautella ZELLER & RE & & & & & \\
\hline \multicolumn{7}{|l|}{ Yponomeutidae } \\
\hline Prayis oleae BERNARD & & & & $\mathrm{RE}$ & & \\
\hline Argyresthia spinosella STAINTON & RE & & & & & \\
\hline Argyresthia hilfiella $\mathrm{REBEL}^{*}$ & & & & $\mathrm{RE}$ & & \\
\hline \multicolumn{7}{|l|}{ Ypsolophidae } \\
\hline Ochsenheimeria taurella D. \& S. & $\begin{array}{c}\text { RE, GOZ } \\
\& \text { ACS }\end{array}$ & & & & & \\
\hline \multicolumn{7}{|l|}{ Plutellidae } \\
\hline Plutella xylostella L. & $\mathrm{RE}$ & & WE & $\mathrm{RE}$ & & \\
\hline \multicolumn{7}{|l|}{ Glyphipterygidae } \\
\hline Glyphipteryx thrasonella Scopoli & & & & & $\mathrm{RE}$ & \\
\hline Glyphipteryx simpliciella STEPHENS & RE & & & & $\mathrm{RE}$ & \\
\hline \multicolumn{7}{|l|}{ Bedelliidae } \\
\hline Bedellia somnulentella ZELLER & $\mathrm{RE}$ & & & & & \\
\hline \multicolumn{7}{|l|}{ Ethmiidae } \\
\hline Ethmia bipunctella $\mathrm{F}$. & STAU & & & & & \\
\hline Ethmia chrysopyga ZELLER & STAU & & & & & \\
\hline Ethmia haemorrhoidella EvERSMANN & STAU & & & & & \\
\hline \multicolumn{7}{|l|}{ Depressariidae } \\
\hline Agonopterx thapsiella ZELLER & $\mathrm{RE}$ & & & & & \\
\hline Agonopterx arenella D. \& S. & STAU & & & & & \\
\hline Agonopterix leucadensis ReBEL * & & RE & & & & \\
\hline Agonopterix irrorata STAUDINGER & $\mathrm{RE}$ & & & $\mathrm{RE}$ & & \\
\hline Depressaria douglasella STAINTON & & & & RE & & \\
\hline Depressaria veneficella ZELLER & STAU & & & & & \\
\hline \multicolumn{7}{|l|}{ Scythrididae } \\
\hline Scythris aerariella H. - S. & STAU & & & & & \\
\hline Scythris punctivittella O. CostA & STAU & & & & & \\
\hline
\end{tabular}




\begin{tabular}{|c|c|c|c|c|c|c|}
\hline Artenliste & Korfu & Lefkada & Ithaka & Kefalonia & Zakynthos & $\begin{array}{c}\text { Kythira/ } \\
\text { Kleine Inseln }\end{array}$ \\
\hline \multicolumn{7}{|l|}{ Oecophoridae } \\
\hline Fabiola pokornyi NICKERL & & & & $\mathrm{RE}$ & & \\
\hline Schiffermuelleria schaefferella L. & & & & RE & & \\
\hline Denisia augustella Нв. & $\mathrm{RE}$ & & & & & \\
\hline Borkhausenia minutella L. & $\mathrm{RE}$ & & & & & \\
\hline Batia lambdella Donovan & STAU & & & & & \\
\hline Epicallima formosella D. \& S. & & RE & & $\mathrm{RE}$ & & \\
\hline Esperia sulphurella $\mathrm{F}$. & GAL & & & WE & & \\
\hline Esperia oliviella $\mathrm{F}$ & STAU & & & & & \\
\hline Harpella foficella SCOPOLI & STAU & & & & & \\
\hline Pleurota marginella D. \& S. & STAU & & & & & \\
\hline Pleurota pyropella D. - S. & $\begin{array}{l}\text { STAU, RE, } \\
\text { GAL }\end{array}$ & & & $\mathrm{RE}$ & $\mathrm{RE}$ & \\
\hline Pleurota pungitiella H. - S. & STAU & & & & & \\
\hline Pleurota aristella L. & STAU & & & & & \\
\hline \multicolumn{7}{|l|}{ Lecithoceridae } \\
\hline Eurodachtha flavissimella MANN & & & & $\mathrm{RE}$ & & \\
\hline \multicolumn{7}{|l|}{ Coleophoridae } \\
\hline Coleophora conyzae ZELLER & BAL & & & & & \\
\hline Coleophora niveicostella ZELLER * & & WE & & & & \\
\hline Coleophora mayrella Нв. & BAL & & & & & \\
\hline Coleophora hieronella ZELLER & $\mathrm{RE}$ & & & & & \\
\hline Coleophora ononidella MiLL. & BAL & & & & & \\
\hline Coleophora texanella CHAMBERs & & & & & LANDRY & \\
\hline \multicolumn{7}{|l|}{ Blastobasidae } \\
\hline Blastobasis phycidella ZELLER & $\mathrm{RE}$ & & & & RE & \\
\hline \multicolumn{7}{|l|}{ Autostichidae } \\
\hline Aprominta designatella H. - S. & $\mathrm{RE}$ & & & & & \\
\hline \multicolumn{7}{|l|}{ Gelechiidae } \\
\hline Isophrictis kefersteiniellus ZELLER & & & & $\mathrm{RE}$ & & \\
\hline Bryotropha basaltinella ZELLER & & & & RE & & \\
\hline Teleiodes vulgella D. \& S. & & & & $\mathrm{RE}$ & & \\
\hline Mirificarma flavella DUPONCHEL & & & & $\mathrm{RE}$ & $\mathrm{RE}$ & \\
\hline Scrobipalpa ocellatella BoyD & & & & & RE & \\
\hline Syncopagma albipalpella $\mathrm{H}$. - S. & STAU & & & & & \\
\hline Anacampsis scintilella F. v. RösLERST. & STAU & & & & & \\
\hline Dicomerus ustalella $\mathrm{F}$. & STAU & & & & & \\
\hline Platyedra subcinerea Haw. & & & & & $\mathrm{RE}$ & \\
\hline \multicolumn{7}{|l|}{ Limacodidae } \\
\hline Apoda limacodes HFN. & STAU & & & & & \\
\hline Hyosia cretica REBEL & & FR \& PI & & & & \\
\hline \multicolumn{7}{|l|}{ Zyganeidae } \\
\hline Jordanita graeca JORDAN & STAU & & & & & \\
\hline Adscita geryon Нв. & STAU & & & & & \\
\hline Adscita mannii LEDERER & $\begin{array}{l}\text { STAU, RE, } \\
\text { HY }\end{array}$ & & & & & \\
\hline Adscita statices $\mathrm{L}$. & STAU & & & & & \\
\hline Zygaena punctum OCHSENHEIMER & $\begin{array}{c}\text { DE LA } \\
\text { GARDE, } \\
\text { STAU, KÖ }\end{array}$ & FR \& PI & & & & \\
\hline Zygaena carniolica SCOPOLI & $\begin{array}{l}\text { STAU, HO, } \\
\text { HY, KÖ }\end{array}$ & & & & & \\
\hline
\end{tabular}




\begin{tabular}{|c|c|c|c|c|c|c|}
\hline Artenliste & Korfu & Lefkada & Ithaka & Kefalonia & Zakynthos & $\begin{array}{l}\text { Kythira/ } \\
\text { Kleine Inseln }\end{array}$ \\
\hline Zygaena ephialtes L. * & $\begin{array}{l}\text { STAU, RE, } \\
\text { HY, KÖ, } \\
\text { EMB, HOF }\end{array}$ & $\begin{array}{l}\text { HOF, FR } \\
\quad \& \mathrm{PI}\end{array}$ & $\mathrm{HOF}$ & HOF, WE & & \\
\hline Zygaena filipendulae L. * & $\begin{array}{c}\text { DE LA } \\
\text { GARDE, } \\
\text { RE, KÖ }\end{array}$ & $\mathrm{RE}$ & WE & RE, WE & EMB & \\
\hline \multicolumn{7}{|l|}{ Zygaena lonicerae SCHEven } \\
\hline \multicolumn{7}{|l|}{ Brachodidae } \\
\hline Brachodes nana TREITsCHKE & STAU & & & & & \\
\hline \multicolumn{7}{|l|}{ Sesiidae } \\
\hline Tinthia tineiformis EsPER & $\begin{array}{l}\text { RE, FR, } \\
\text { PRE }\end{array}$ & & & FR? & EMB & \\
\hline Tinthia brosiformis Нв. & STAU, FR & & & & & \\
\hline Tinthia myrmosaeformis H. - S. & FR & & & FR? & FR? & \\
\hline Sesia apiformis CLERCK & FR & & & FR? & FR? & \\
\hline $\begin{array}{l}\text { Paranthrene tabaniformnis } \\
\text { ROTTEMBURG }\end{array}$ & FR & & & FR? & EMB & \\
\hline Synanthedon culiciformis $\mathrm{L}$. & FR & & & & & \\
\hline Synanthedon vespiformis L. & STAU, FR & & & FR? & FR? & \\
\hline $\begin{array}{l}\text { Synanthedon myopaeformis } \\
\text { BORKHAUSEN }\end{array}$ & RE, FR & & & FR ? & FR? & \\
\hline Synanthedon conopiformis EsPER & FR & & & FR? & FR? & \\
\hline Synanthedon tipuliformis CLERCK & FR & & & & & \\
\hline Synanthedon spuleri FucHs & FR & & & FR ? & FR? & \\
\hline Bembecia ichneumoniformis D. \& S. & STAU, FR & & & FR? & FR? & \\
\hline Bembecia albanensis REBEL & $\begin{array}{c}\text { STAU, RE, } \\
\text { SP \& LA, } \\
\text { FR }\end{array}$ & & & FR? & FR? & \\
\hline Bembecia pavicevici Tosevski & SP, FR & & & FR? & FR, PÜH & Paxos $=\mathrm{EMB}$ \\
\hline Bembecia fokidensis TosEVsKI & & & & & PÜH & \\
\hline Bembecia uroceriformis TREITSCHKE & STAU, FR & & & FR? & FR? & \\
\hline Pyropteron minianiformis FREYER & $\begin{array}{l}\text { STAU, RE, } \\
\text { FR }\end{array}$ & & & FR? & EMB & \\
\hline Synansphecia triannuliformis FREYER & $\begin{array}{l}\text { STAU, LA, } \\
\text { FR }\end{array}$ & & & FR? & FR? & \\
\hline Synansphecia muscaeformis EsPER & FR & & & & & \\
\hline Synansphecia affinis STAUDINGER & STAU, FR & & & FR? & FR? & \\
\hline Synansphecia leucomelaena ZELLER & FR & & & FR? & FR? & \\
\hline Synansphecia umbrifera STAUDINGER * & $\begin{array}{l}\text { STAU, RE, } \\
\text { FR }\end{array}$ & & & & & \\
\hline Chamaesphecia alysoniformis H. - S. & FR & & & FR ? & FR? & \\
\hline Chamaesphecia chalciformis EsPER & FR & & & FR? & FR? & \\
\hline $\begin{array}{l}\text { Chamaesphecia schmidtiiformis } \\
\text { FREYER }\end{array}$ & FR & & & FR? & FR? & \\
\hline Chamaesphecia doleriformis H. - S. & STAU, FR & & & & & \\
\hline $\begin{array}{l}\text { Chamaesphecia proximata } \\
\text { STAUDINGER }\end{array}$ & FR & & & & & \\
\hline Chamaeshecia annellata ZELLER & STAU, FR & & & & & \\
\hline $\begin{array}{l}\text { Chamaesphecia masariformis } \\
\text { OCHSENHEIMER }\end{array}$ & STAU, FR & & & & & \\
\hline Chamaesphecia empiformis EsPER & STAU, FR & & & & & \\
\hline
\end{tabular}




\begin{tabular}{|c|c|c|c|c|c|c|}
\hline Artenliste & Korfu & Lefkada & Ithaka & Kefalonia & Zakynthos & $\begin{array}{c}\text { Kythira/ } \\
\text { Kleine Inseln }\end{array}$ \\
\hline \multicolumn{7}{|l|}{ Cossidae } \\
\hline Dyspessa ulula Borkhausen & & $\begin{array}{l}\text { WE, FR } \\
\& \text { PI }\end{array}$ & WE & RE, WE & & \\
\hline \multicolumn{7}{|l|}{ Tortricidae } \\
\hline Obraztsoviana maculosana HAWORTH ${ }^{*}$ & & & & WE & & \\
\hline Cochylimorpha straminea HAWORTH & STAU & & & & & \\
\hline Aethes tesserana D. \& S. & STAU & & & & & \\
\hline Aethes francillana $\mathrm{F}$. & STAU & & & & & \\
\hline Acleris variegana $\mathrm{D}$. - S. & $\mathrm{RE}$ & & & & & \\
\hline Acleris aspersana D. - S. & $\mathrm{RE}$ & & & & & \\
\hline Acleris notana Donovan & & & & $\mathrm{RE}$ & & \\
\hline Acleris lipsiana D. \& S. & STAU & & & & & \\
\hline Tortricodes alternella D. - S. ${ }^{*}$ & & & & WE & & \\
\hline Cnephasia incertana TREITsChKe & $\mathrm{RE}$ & & & $\mathrm{RE}$ & $\mathrm{RE}$ & \\
\hline Cnephasia abrasana Duponchel & $\mathrm{RE}$ & & & & & \\
\hline Cnephasia pasiuana Нв. & STAU & & & $\mathrm{RE}$ & & \\
\hline $\begin{array}{l}\text { Cnephasia cupressivorana } \\
\text { STAUDINGER * }\end{array}$ & & & & WE & & \\
\hline Cnephasia oxyacanthana H. - S. & & $\mathrm{RE}$ & & $\mathrm{RE}$ & & \\
\hline Cnephasia longana HAw. & $\begin{array}{l}\text { STAU, } \\
\text { DE LA } \\
\text { GARDE }\end{array}$ & & & & & \\
\hline Cnephasia gueneana DuPONCHEL & STAU & & & & & \\
\hline Sparganothes pilleriana D. \& S. & STAU & & & & & \\
\hline Avaria hyerana MiLL. & $\mathrm{RE}$ & & & & & \\
\hline Archips rosana $\mathrm{L}$. & STAU & & & & & \\
\hline Cacoecimorpha pronubana Нв. & STAU & & & & $\mathrm{RE}$ & \\
\hline Aphelia amplana Нв. & $\mathrm{RE}$ & & & & RE & \\
\hline Clepsis steineriana Нв. & STAU & & & & & \\
\hline Clepsis consimilana Нв. & $\mathrm{RE}$ & & & & & \\
\hline Endothenia sororiana H. - S. & STAU, RE & & & & & \\
\hline Pseudosciaphila branderiana L. & $\mathrm{RE}$ & & & & & \\
\hline Lobesia botrana D. \& S. ${ }^{*}$ & & & WE & & & \\
\hline Epinotia thapsiana ZELLER * & STAU & & WE & & & \\
\hline Crocidosema plebejana ZeLLeR & & $\mathrm{RE}$ & & & & \\
\hline Eucosma conformana MANN & & & & $\mathrm{RE}$ & & \\
\hline Notocelia uddmanniana L. & & & & $\mathrm{RE}$ & & \\
\hline Blastesthia tessulatana STAUDINGER * & & & WE & WE & & \\
\hline Cydia ulicetana Haw. & & & & & $\mathrm{RE}$ & \\
\hline Selania capparidana ZELLER & RE, GOZ & & & & & \\
\hline Dichrorampa petiverella $\mathrm{L}$. & $\mathrm{RE}$ & & & & & \\
\hline Phalonidia albipalpana ZeLLER * & & GRAF & & & & \\
\hline Millieria dolosalis HEYDENREICH & & & & $\mathrm{RE}$ & & \\
\hline Prochoreutis stellaris ZELLER * & FRIE & & & & & \\
\hline Choreutis nemorana Нв. & $\mathrm{RE}$ & & & & & \\
\hline \multicolumn{7}{|l|}{ Alucitidae } \\
\hline Alucita cymatodactyla ZELLER & & & & $\mathrm{RE}$ & & \\
\hline Alucita grammodactyla ZeLLER & & & & $\mathrm{RE}$ & & \\
\hline Alucita desmodactyla ZELLER & & $\mathrm{RE}$ & & & & \\
\hline \multicolumn{7}{|l|}{ Pterophoridae } \\
\hline Agdistis frankeniae ZELLER & AR & & & & & \\
\hline
\end{tabular}




\begin{tabular}{|c|c|c|c|c|c|c|}
\hline Artenliste & Korfu & Lefkada & Ithaka & Kefalonia & Zakynthos & $\begin{array}{c}\text { Kythira/ } \\
\text { Kleine Inseln }\end{array}$ \\
\hline Agdistis paralia ZELLER & STAU & & & & & \\
\hline Amblyptilia acanthadactyla Нв. & $\mathrm{RE}$ & & & & & \\
\hline $\begin{array}{l}\text { Stenoptilia zophodactylus } \\
\text { DuPONCHEL }\end{array}$ & $\mathrm{RE}$ & & & & & \\
\hline Oxyptilus distans ZELLER * & & & WE & & & \\
\hline Stangeia siceliota ZELLER * & & & WE & & & \\
\hline Pterophorus pentadactyla L. * & & & & WE & & \\
\hline Merrifieldia malacodactylus ZeLLeR & & & WE & $\mathrm{RE}$ & & \\
\hline Euleioptilus distinctus H. - S. & & & & & $\mathrm{RE}$ & \\
\hline Emmelina monadactyla L. & $\mathrm{RE}$ & & & & & \\
\hline \multicolumn{7}{|l|}{ Pyralidae } \\
\hline Aphomia zelleri JoAnNIs & & & & & RE, EMB & \\
\hline Amoria anella D. \& S. & & $\mathrm{RE}$ & & & EMB & \\
\hline Hypotia infulalis LEDERER * & & GRAF & & & & \\
\hline Corcyra cephalonica STAINTON & $\mathrm{RE}$ & & & & & \\
\hline Synaphe moldavica ESPER & $\begin{array}{l}\text { STAU, } \\
\text { GINZ }\end{array}$ & RE & & RE & EMB & \\
\hline Pyralis obsoletalis MANN & & $\mathrm{RE}$ & & & & \\
\hline Pyralis farinalis L. & $\begin{array}{l}\text { DE LA } \\
\text { GARDE }\end{array}$ & & & & & \\
\hline Aglossa pinguinalis $\mathrm{L}$. & STAU & & & & & \\
\hline Endotricha flammealis D. \& S. & & RE & & & EMB & $\begin{array}{c}\text { Meganisi }= \\
\text { RE }\end{array}$ \\
\hline Oncocera semirubella SCOPOLI * & DAHL & & & & & \\
\hline Pempelia palumbella D. \& S. & STAU & & & & & \\
\hline Pempelia amoenella ZELLER & STAU & & & & & \\
\hline Eurhodope incompta ZELLER & & & & RE & & \\
\hline Asalebria florella MANN & STAU, RE & & & & & \\
\hline Eccopisa effractella ZELLER & $\mathrm{RE}$ & & & & & \\
\hline Euzophera bigella ZELLER & & & & & RE, GOZ & \\
\hline Euzophera lunulella O. CosTA & STAU & & & & & \\
\hline Euzopherodes lutisignella MANN & & & & RE & & \\
\hline Ancylosis cinnamomella DUPONCHEL & STAU & & & & & \\
\hline Ancylosis hellenica STAUdINGER & $\mathrm{RE}$ & & & & & \\
\hline Homoeosama sinuella $\mathrm{F}$ & STAU & & & & & \\
\hline Ephestia elutella Нв. & & & & & $\mathrm{RE}$ & \\
\hline Raphimetopus nitidicostella RAGONOT & & $\mathrm{RE}$ & & & & \\
\hline Epidauria transversariella ZELLER & $\begin{array}{l}\text { STAU, } \\
\text { GOZ }\end{array}$ & & & & & \\
\hline Epidauria strigosa STAUDINGER & RE, GAL & & & & & \\
\hline Ematheudes punctella TREIтschKe & $\begin{array}{l}\text { STAU, } \\
\text { GAL, BLA }\end{array}$ & & & & & \\
\hline \multicolumn{7}{|l|}{ Crambidae } \\
\hline Eudonia angustea CuRTIS & RE & & & & & \\
\hline Eucromius ocellea HAw. & STAU, RE & & & & $\mathrm{RE}$ & \\
\hline Eucromius bella Нв. & STAU & & & & & \\
\hline Eucromius superbellus ZELLER * & & & & & EMB & \\
\hline Crambus inquinatellus D. \& S. & STAU & & & & & \\
\hline Metacrambus carectellus ZeLLER & $\mathrm{RE}$ & & & & & \\
\hline Crysocrambus craterella Scopoli & KRU & & & $\mathrm{RE}$ & & \\
\hline Elophila nymphaeata L. & & RE, SPEI & & & & \\
\hline Elophila rivulalis DUPONCHEL & & RE, SPEI & & & & \\
\hline
\end{tabular}




\begin{tabular}{|c|c|c|c|c|c|c|}
\hline Artenliste & Korfu & Lefkada & Ithaka & Kefalonia & Zakynthos & $\begin{array}{l}\text { Kythira/ } \\
\text { Kleine Inseln }\end{array}$ \\
\hline Cataclysta lemnata L. & & RE, SPEI & & & & \\
\hline Hydriris ornatalis DuPONCHEL * & DAHL & & & & & \\
\hline Aporodes floralis Нв. & $\begin{array}{c}\text { STAU, } \\
\text { GAL, FRIE }\end{array}$ & & & & & \\
\hline Evergestis extimalis SCOPOLI & STAU & & & & & \\
\hline Udea ferrugalis Нв. & $\mathrm{RE}$ & & & & $\mathrm{RE}$ & \\
\hline Udea fulvalis Нв. & STAU & & & $\mathrm{RE}$ & EMB & \\
\hline Achyra nudalis Нв. & $\mathrm{RE}$ & & & & & \\
\hline Ecpyrrhorrhoe rubiginalis Нв. & STAU & & & & & \\
\hline Pyrausta sanguinalis L. & & & & $\mathrm{RE}$ & & \\
\hline Pyrausta despicata SCOPOLI & & $\mathrm{RE}$ & & & & $\begin{array}{c}\text { Meganisi }= \\
\text { RE }\end{array}$ \\
\hline Pyrausta aurata Scopoli & $\mathrm{RE}$ & & & & & \\
\hline Pyrausta purpuralis L. & $\begin{array}{c}\text { DE LA } \\
\text { GARDE }\end{array}$ & & & & & \\
\hline Pyrausta pygmaealis DUPONCHEL & STAU & & & & & \\
\hline Uresiphita gilvata $\mathrm{F}$. & STAU & & & & & \\
\hline Sitochroa palaelis D. \& S. & STAU & & & & & \\
\hline Phlyctaenia stachydalis GERMAR & STAU & & & & & \\
\hline Ebulea testacealis ZELLER & STAU & & & & & \\
\hline Anania verbascalis D. \& S. & STAU & & & & & \\
\hline Mercyna flavalis D. \& S. & STAU & & & & & \\
\hline Mecyna asinalis Нв. ${ }^{*}$ & KRU & & & & & \\
\hline Duponchelia fovealis ZELLER & $\begin{array}{c}\text { DE LA } \\
\text { GARDE }\end{array}$ & & & & & \\
\hline Palpita vitrealis Rossi ${ }^{*}$ & DAHL & & & & & \\
\hline Amaurophanes stigmosalis H. - S. & & & & $\mathrm{RE}$ & & \\
\hline Dolicharthria punctalis D. \& S. & & $\mathrm{RE}$ & & & & \\
\hline $\begin{array}{l}\text { Dolicharthria bruguieralis } \\
\text { DuPONCHEL }\end{array}$ & $\mathrm{RE}$ & $\mathrm{RE}$ & & & & $\underset{\text { RE }}{\text { Meganisi }}=$ \\
\hline Metasia suppandalis Нв. & STAU & & & & & \\
\hline Metasia ophialis TreITSCHKE * & & & WE & & & \\
\hline Nomophila noctuella D. \& S. & $\begin{array}{l}\text { STAU, RE, } \\
\text { GAL, BLA }\end{array}$ & $\mathrm{RE}$ & & $\mathrm{RE}$ & & \\
\hline \multicolumn{7}{|l|}{ Lasiocampidae } \\
\hline Malacosoma neustria L. * & & $\begin{array}{l}\text { WE, FR } \\
\text { \& PI }\end{array}$ & WE & WE & & \\
\hline Lasiocampa trifolii D. \& S. & STAU, MA & WE & & RE, MA & & MA \\
\hline Lasiocampa quercus L. & MA & & WE & WE & & \\
\hline \multicolumn{7}{|l|}{ Saturniidae } \\
\hline Saturnia pyri D. \& S. & & & & & & $\mathrm{RE}$ \\
\hline Saturnia pavonia L. & STAU & & & & & \\
\hline \multicolumn{7}{|l|}{ Lemoniidae } \\
\hline Lemonia taraxaci D. \& S. * & & & WE & & & \\
\hline \multicolumn{7}{|l|}{ Sphingidae } \\
\hline Marumba quercus D. \& S. * & & FR \& PI & & WE & PAPE & \\
\hline Smerinthus ocellatus L. & & FR \& PI & & & & \\
\hline Agrius convolvuli L. & & FR \& PI & & & & \\
\hline Acheronta atropos L. & & FR \& PI & & & & \\
\hline Macroglossum stellatarum L. & $\mathrm{RE}$ & & WE & WE & & \\
\hline Daphnis nerii L. & STAU & & & & & \\
\hline Hyles euphorbiae L. * & STAU ? & & & WE & & \\
\hline
\end{tabular}




\begin{tabular}{|c|c|c|c|c|c|c|}
\hline Artenliste & Korfu & Lefkada & Ithaka & Kefalonia & Zakynthos & $\begin{array}{l}\text { Kythira/ } \\
\text { Kleine Inseln }\end{array}$ \\
\hline Hyles livornica EsPER & STAU & WE & WE & WE & EMB & $\mathrm{RE}$ \\
\hline Deilephila porcellus L. & & FR \& PI & & & & \\
\hline Theretra alecto $\mathrm{L}$. & STAU ? & & & & & \\
\hline \multicolumn{7}{|l|}{ Hesperiidae } \\
\hline Erynnis tages $\mathrm{L}$. & PA & & & & & \\
\hline Erynnis marloyi BOISDUVAL & PA & & & & & \\
\hline Carcharodes alceae EsPER & $\begin{array}{l}\text { PA, PARK, } \\
\text { KLEW }\end{array}$ & & WE & PA & & PA \\
\hline Carcharodes lavatherae EsPER & STAU, PA? & & & & & $\begin{array}{c}\mathrm{KA} \& \mathrm{KO} \\
\mathrm{PA} ?\end{array}$ \\
\hline Carcharodes floccifera ZELLER & STAU, PA ? & & & & & \\
\hline Carcharodes orientalis REVERDIN & $\begin{array}{l}\text { PA, PARK, } \\
\text { FRIE }\end{array}$ & & & PA & & \\
\hline Spialia orbifer Нв. & PA, EMB, & PA, STR & WE & PA, WE & PA & \\
\hline Muschampia proto OCHSENHEIMER * & PA, PARK & & WE & WE & & $\begin{array}{c}\text { PA/MAT = } \\
\text { Vido }\end{array}$ \\
\hline Pyrgus malvae L. & PA & & WE & WE & & \\
\hline Pyrgus armoricanus OBERTHÜR & & & & & & $\mathrm{PA}$ \\
\hline Thymelicus sylvestris PODA & PA, GINZ & WE & WE & $\mathrm{PA}, \mathrm{WE}$ & & $\mathrm{PA}$ \\
\hline Thymelicus acteon RotTEMBURG & PA & PA & & PA & PA & $\mathrm{PA}$ \\
\hline Ochlodes sylvanus EsPer & PA, PARK & & & & PA & \\
\hline Gegenes pumilio HOFFMANNSEGG * & PA, PARK & & WE & & EMB & $\begin{array}{c}\text { PA/MAT = } \\
\text { Vido }\end{array}$ \\
\hline \multicolumn{7}{|l|}{ Papilionidae } \\
\hline Zerynthia polyxena D. \& S. & PA & PA & & $\mathrm{PA}$ & PA & \\
\hline Iphiclidis podalirius L. & PA, PARK & $\mathrm{PA}, \mathrm{WE}$ & WE & $\mathrm{PA}, \mathrm{WE}$ & PA & $\begin{array}{c}\mathrm{PA} / \mathrm{PA}= \\
\text { Paxos }\end{array}$ \\
\hline Papilio machaon L. & PA, PARK & $\mathrm{PA}, \mathrm{WE}$ & WE & $\mathrm{PA}, \mathrm{WE}$ & PA & $\mathrm{PA}$ \\
\hline Papilio alexanor ESPER & PA & PA & & PA & PA & \\
\hline \multicolumn{7}{|l|}{ Pieridae } \\
\hline Leptidea sinapis $\mathrm{L}$. & PA, PARK & $\mathrm{PA}, \mathrm{WE}$ & $\begin{array}{c}\text { PAP\& } \\
\text { KA, PA, } \\
\text { WE }\end{array}$ & $\mathrm{PA}, \mathrm{WE}$ & PA & \\
\hline Leptidea duponcheli STAUDINGER & PA? & & & & PA & \\
\hline Anthocharis cardaminis L. & PA & $\mathrm{PA}, \mathrm{WE}$ & WE & $\begin{array}{c}\text { PAP\&KA, } \\
\text { PA, WE }\end{array}$ & & \\
\hline Anthocharis damone BOISDUVAL & PA & & & & & \\
\hline Euchloe ausonia Нв. & PA & PA & WE & $\mathrm{PA}, \mathrm{WE}$ & PA & $\mathrm{PA}$ \\
\hline Aporia crataegi $\mathrm{L}$. & & PA, WE & WE & PA, WE & & PA \\
\hline Pieris brassicae $\mathrm{L}$. & PA, PARK & PA, WE & WE & PA, WE & PA & PA \\
\hline Pieris krueperi STAUDINGER & PA & & & & & \\
\hline Pieris mannii MAYER & $\begin{array}{l}\text { PA, PARK, } \\
\text { VO, GINZ }\end{array}$ & & & & & \\
\hline Pieris rapae $\mathrm{L}$. & PA, PARK & WE & WE & PA, WE & PA & PA \\
\hline Pieris ergane GEYER & PA & & & $\begin{array}{c}\text { PAP\&KA, } \\
\text { PA }\end{array}$ & & \\
\hline Pieris napi L. & PA & & & & & \\
\hline Pieris balcana LoRCOVIC & GINZ & & & & & \\
\hline Pontia edusa F. & PA, PARK & PA & WE & $\begin{array}{c}\text { PAP\&KA, } \\
\text { PA, WE }\end{array}$ & & PA \\
\hline Colias croceus Fourc. & PA, PARK & $\mathrm{PA}, \mathrm{WE}$ & WE & PA, WE & PA & PA \\
\hline
\end{tabular}




\begin{tabular}{|c|c|c|c|c|c|c|}
\hline Artenliste & Korfu & Lefkada & Ithaka & Kefalonia & Zakynthos & $\begin{array}{c}\text { Kythira/ } \\
\text { Kleine Inseln }\end{array}$ \\
\hline Colias alfacariensis RIBBE* & $\mathrm{PA}$ & & & PA?, WE & PA? & \\
\hline Gonepteryx rhamni L. & PA & PA & WE & PA, WE & PA & \\
\hline Gonepteryx farinosa ZELLER & PA? & PA & & PA & EMB & \\
\hline Gonepteryx cleopatra L. & PA, PARK & $\mathrm{PA}, \mathrm{WE}$ & WE & PA, WE & PA & $\begin{array}{l}\mathrm{PA} / \mathrm{PA}= \\
\text { Paxos }\end{array}$ \\
\hline \multicolumn{7}{|l|}{ Lycaenidae } \\
\hline Lycaena phlaeas L. & PA, PARK & PA & WE & PA, WE & PA & PA \\
\hline Lycaena ottomanus LEFEBVRe & PA, DAHL & & & & & \\
\hline Lycaena alciphron ROTTEMBURG & PA & & & & & \\
\hline Lycaena thersamon EsPER & $\mathrm{PA}$ & & & & & \\
\hline Favonius quercus L. & PA & & & $\begin{array}{l}\text { PAP\&KA, } \\
\text { PA }\end{array}$ & & \\
\hline Callophrys rubi L. & $\mathrm{PA}$ & PA, WE & WE & PA, WE & PA & PA \\
\hline Satyrium spini D. \& S. & PA & PA & & PA & PA & PA \\
\hline Satyrium ilicis ESPER & PA, GINZ & & WE & PA & & PA \\
\hline Satyrium acaciae $\mathrm{F}$. & PA & & & & & \\
\hline Lampides boeticus $\mathrm{L}$. & PA, PARK & PA & WE & PA & PA & $\mathrm{PA}$ \\
\hline Cacyreus marshalli ButLER & PA, PARK & & & & & \\
\hline Leptotes pirithous L. & $\begin{array}{l}\text { PA, PARK, } \\
\text { KLEW }\end{array}$ & & & PA & & PA \\
\hline Tarucus balkanica FreYer & PA & PA? & & & & \\
\hline Cupido minimus Fuessly & PA & & & & & \\
\hline Celastrina argiolus L. & PA, PARK & PA & WE & $\mathrm{PA}, \mathrm{WE}$ & PA & PA \\
\hline Pseudophilotes vicrama MoORE & PA & PA & WE & PA & $\mathrm{PA}$ & $\mathrm{PA}$ \\
\hline Scolitantides orion PALLAS * & STAU, PA? & & WE & & & \\
\hline Glaucopsyche alexis PoDA & PA & $\mathrm{PA}, \mathrm{WE}$ & WE & $\mathrm{PA}, \mathrm{WE}$ & $\mathrm{PA}$ & $\mathrm{PA}$ \\
\hline Chilades trochylus Freyer & $\mathrm{PA}$ & & & & & \\
\hline Plebejus argus L. & PA & & & & & \\
\hline Aricia agestis D. \& S. & PA, PARK & & WE & PA & PA & PA \\
\hline $\begin{array}{l}\text { Polyommatus semiargus } \\
\text { RotTEMBURG }\end{array}$ & PA & & & & & $\begin{array}{l}\text { KA \& KO, } \\
\text { PA ? }\end{array}$ \\
\hline Polyommatus dorylas D. \& S. & STAU, PA? & & & STAU & & \\
\hline Polyommatus thersites CANTENER & PA & $\mathrm{PA}$ & & PA & & \\
\hline Polyommatus icarus RotTEMBURG & PA, PARK & $\mathrm{PA}$ & WE & $\mathrm{PA}$ & $\mathrm{PA}$ & $\mathrm{PA}$ \\
\hline \multicolumn{7}{|l|}{ Nymphalidae } \\
\hline Libythea celtis LAICH. & PA, GINZ & & & & & \\
\hline Argynnis paphia L. & PA, PARK & & & $\mathrm{PA}$ & & \\
\hline Argynnis pandora D. \& S. & PA & & & & & \\
\hline Issoria lathonia L. * & PA & & & $\begin{array}{l}\text { PAP\&KA, } \\
\text { PA, WE }\end{array}$ & PA ? & \\
\hline Vanessa atalanta $\mathrm{L}$. & PA, PARK & PA & WE & PA, WE & PA & $\mathrm{PA}$ \\
\hline Vanessa cardui L. & PA, PARK & PA, WE & WE & PA, WE & PA & PA \\
\hline Aglais io L. & PA & & & & & \\
\hline Aglais urticae L. & PA & & & & & \\
\hline Polygonia c-album L. & PA & & WE & & & \\
\hline Polygonia egea CRAMER & PA, GINZ & & WE & $\mathrm{PA}, \mathrm{WE}$ & PA & $\mathrm{PA}$ \\
\hline Nymphalis antiopa $\mathrm{L}$. & $\mathrm{PA}$ & & & & & \\
\hline Nymphalis polychloros L. & $\mathrm{PA}$ & & WE & $\mathrm{PA}$ & & \\
\hline Melitaea cinxia L. & $\mathrm{PA}$ & & & & & \\
\hline Melitaea phoebe D. \& S. & PA & & & & & \\
\hline Melitaea trivia D. \& S. & STAU, PA? & & & & & \\
\hline Melitaea didyma EsPER & PA, PARK & PA & & $\mathrm{PA}$ & PA & \\
\hline
\end{tabular}




\begin{tabular}{|c|c|c|c|c|c|c|}
\hline Artenliste & Korfu & Lefkada & Ithaka & Kefalonia & Zakynthos & $\begin{array}{c}\text { Kythira/ } \\
\text { Kleine Inseln }\end{array}$ \\
\hline Liminitis reducta STAUDINGER & PA, PARK & PA & WE & PA, WE & PA & PA \\
\hline Charaxes jasius L. & PA, PARK & & $\mathrm{PA}$ & PA & & \\
\hline Kirinia roxelana CRAMER & PA & PA & WE & PA & & PA \\
\hline Pararge aegeria $\mathrm{L}$. & PA, PARK & & & $\mathrm{PA}$ & PA & $\mathrm{PA}$ \\
\hline Lasiommata megera $\mathrm{L}$. & PA, PARK & $\mathrm{PA}, \mathrm{WE}$ & WE & $\mathrm{PA}, \mathrm{WE}$ & $\mathrm{PA}$ & $\mathrm{PA}$ \\
\hline Lasiommata maera L. & PA & $\mathrm{PA}, \mathrm{WE}$ & WE & $\mathrm{PA}, \mathrm{WE}$ & & \\
\hline Coenonympha pamphilus L. & PA, PARK & $\mathrm{PA}, \mathrm{WE}$ & WE & $\mathrm{PA}, \mathrm{WE}$ & PA & RE, PA? \\
\hline Pyronia cecilia Vallantin & $\mathrm{PA}$ & PA & & & & \\
\hline Maniola jurtina $\mathrm{L}$. & PA, PARK & $\mathrm{PA}, \mathrm{WE}$ & WE & $\mathrm{PA}, \mathrm{WE}$ & $\mathrm{PA}$ & $\mathrm{PA}$ \\
\hline Hyponephele lycaon RotTEMBURG & PA? & & $\begin{array}{l}\text { PAP\& } \\
\text { KA?, PA }\end{array}$ & & & \\
\hline Hyponephele lupinus О. СоsтА & $\mathrm{PA}$ & & & $\begin{array}{c}\text { PAP\&KA } \\
\text { ?, PA }\end{array}$ & & $\mathrm{PA}$ \\
\hline Melanargia galathea L. & PA & & & & & \\
\hline Melanargia larissa GEYER & $\mathrm{PA}$ & $\mathrm{PA}$ & & & & \\
\hline Hipparchia fagi SCOPOLI & PA & PA & WE & $\begin{array}{l}\text { PAP\&KA, } \\
\text { PA }\end{array}$ & & PA? \\
\hline Hipparchia syriaca STAUDINGER & PA, PARK & $\mathrm{PA}$ & & $\mathrm{PA}$ & & $\mathrm{PA}$ \\
\hline Hipparchia senthes FrUHSTORFER & $\mathrm{PA}, \mathrm{HU}$ & $\mathrm{PA}$ & $\mathrm{PA} ?$ & $\mathrm{PA}$ & & $\mathrm{PA}$ \\
\hline $\begin{array}{l}\text { Hipparchia volgensis MAzOCHIN- } \\
\text { PORshN. }\end{array}$ & PA, PARK & & & $\begin{array}{l}\text { PAP\&KA, } \\
\text { PA }\end{array}$ & $\mathrm{PA}$ & \\
\hline Hipparchia statilinus HFN. & PA, PARK & & WE & $\begin{array}{l}\text { PAP\&KA, } \\
\text { PA }\end{array}$ & & $\mathrm{PA}$ \\
\hline Hipparchia fatua FrEYER & & PA & & $\begin{array}{l}\text { PAP\&KA, } \\
\text { PA }\end{array}$ & $\mathrm{PA}$ & PA \\
\hline Brintesia circe $\mathrm{F} . *$ & PA & & WE & & & \\
\hline Danaus chrysippus L. & $\mathrm{PA}$ & $\mathrm{PA}$ & & $\begin{array}{l}\text { PAP\&KA, } \\
\text { PA }\end{array}$ & & \\
\hline \multicolumn{7}{|l|}{ Drepanidae } \\
\hline Thyatira batis $\mathrm{L}$. & STAU & & & & & \\
\hline Habrosyne pyritoides HFN. ${ }^{*}$ & DAHL & & & & & \\
\hline Polyploca ridens $\mathrm{F} .{ }^{*}$ & & & WE & & & \\
\hline Falcaria lacertinaria $\mathrm{L}$. & STAU & & & & & \\
\hline Watsonalla uncinula BORKHAUSEN * & & $\begin{array}{l}\text { FR \& PI, } \\
\text { WE }\end{array}$ & WE & WE & PAPE & \\
\hline Cilix glaucata SCOPOLI & & FR \& PI & WE & MA, WE & & \\
\hline \multicolumn{7}{|l|}{ Geometridae } \\
\hline Ligdia adustata D. \& S. & STAU & & & & & \\
\hline Godonella aestimaria Нв. * & $\begin{array}{l}\text { STAU, } \\
\text { EMB }\end{array}$ & & & & & \\
\hline $\begin{array}{l}\text { Neognopharmia stevenaria } \\
\text { BoIsDUVAL * }\end{array}$ & & & WE & & & \\
\hline Rhoptria asperaria Нв. ${ }^{\star}$ & & & WE & WE & & \\
\hline Rhoptria dolosaria H. - S. & RE & & & & & \\
\hline Ortariella gruneraria STAUDINGER & & $\mathrm{RE}$ & & & & \\
\hline Pachicnemia hippocastanataria Нв. ${ }^{*}$ & & & WE & WE & & \\
\hline Dasycorsa modesta STAUDINGER & & & & SK \& SI & & \\
\hline Asovia maeoticaria AlPHERAKY & & & & & & SK \& SI \\
\hline Lycia graecarius STAUDINGER & STAU & RE & & & & \\
\hline Nychiodes dalmatina WAGNER * & & & WE & & & \\
\hline Menophra abruptaria THNBG. * & & WE & WE & WE & & \\
\hline Menophra japygiaria O. Costa & $\mathrm{RE}$ & & & & & \\
\hline
\end{tabular}




\begin{tabular}{|c|c|c|c|c|c|c|}
\hline Artenliste & Korfu & Lefkada & Ithaka & Kefalonia & Zakynthos & $\begin{array}{c}\text { Kythira/ } \\
\text { Kleine Inseln }\end{array}$ \\
\hline Menophra berenicidaria Turati ${ }^{*}$ & & & WE & & EMB & \\
\hline Peribatodes rhomboidaria D. \& S. & $\mathrm{RE}$ & WE & WE & WE & & \\
\hline Peribatodes umbraria Нв. * & $\mathrm{VO}$ & WE & WE & WE & & \\
\hline Peribatodes correptaria ZELLER * & $\begin{array}{l}\text { DAHL, } \\
\text { STRU }\end{array}$ & WE & WE & WE & & \\
\hline Ascotis selenaria D. \& S. ${ }^{\star}$ & & & WE & WE & & \\
\hline Ectropis crepuscularia D. \& S. * & DAHL & & & & & \\
\hline Ematurga atomaria L. ${ }^{*}$ & DAHL & & & & & \\
\hline Campaea honoraria D. \& S. * & & & WE & WE & & \\
\hline Pungeleria capreolaria D. \& S. * & & & & $\mathrm{RE}$ & & \\
\hline Kemtrognophos onustaria H. - S. * & & WE & WE & & & \\
\hline Euchrognophos variegata DuP. & & & WE & RE, WE & & \\
\hline Dicrognophos sartata TREITSCHKE & & $\mathrm{RE}$ & WE & RE, WE & & $\begin{array}{c}\text { Meganisi }= \\
\text { RE }\end{array}$ \\
\hline Aspitates ochrearia Rossi & STAU, RE & $\begin{array}{c}\text { RE, SK \& } \\
\text { SI, WE }\end{array}$ & WE & WE & $\mathrm{RE}$ & \\
\hline Aplasta ononaria FuessLy & RE, HAU & & & & & \\
\hline Pseudoterpna pruinata HFN. ${ }^{*}$ & STAU & & WE & & & \\
\hline Phaiogramma etruscaria ZELLER & & RE, HAU & WE & $\begin{array}{c}\text { RE, HAU, } \\
\text { WE }\end{array}$ & RE, HAU & \\
\hline Eucrostis indigenata VILLERS * & & $\begin{array}{l}\text { WE, } \\
\text { GRAF }\end{array}$ & & & & \\
\hline Xenochlorodes olympiaria H. - S. & & & WE & WE & $\begin{array}{l}\text { HAU, } \\
\text { PAPE }\end{array}$ & \\
\hline Cyclophora puppillaria Нв. & $\begin{array}{l}\text { RE, HAU, } \\
\text { DAHL }\end{array}$ & WE & WE & WE & RE, EMB & \\
\hline Cosymbia suppunctaria ZeLLER * & & & & WE & & \\
\hline Scopula ornata Scopoli & STAU & & & & & \\
\hline Scopula submutata TreitschKe * & & & WE & WE & & \\
\hline Scopula vigilata WAGNER * & & & WE & & & \\
\hline Scopula decorata D. \& S. & & & & $\mathrm{HAU}$ & & \\
\hline Scopula rubiginata HFN. ${ }^{*}$ & DAHL & & & & & \\
\hline Scopula marginepunctata GoEzE * & & & WE & $\mathrm{WE}$ & & \\
\hline Scopula imitaria Нв. * & $\begin{array}{c}\text { STAU, } \\
\text { DE LA } \\
\text { GARDE, } \\
\text { RE, KRU }\end{array}$ & & WE & RE, WE & RE, PAPE & \\
\hline Scopula flaccidaria ZELLER & $\begin{array}{l}\text { STAU, } \\
\text { DAHL }\end{array}$ & & & & & \\
\hline Scopula minorata BoIsDuvaL * & $\begin{array}{l}\text { EMB, } \\
\text { HAU }\end{array}$ & & WE & & EMB & \\
\hline Glossotrophia confinaria H. - S. * & STAU & & WE & & & \\
\hline Idaea ochrata Scopoli & $\begin{array}{c}\text { STAU, } \\
\text { HAU, KRU }\end{array}$ & & & & EMB & \\
\hline Idaea rusticata D. \& S. & $\begin{array}{c}\text { STAU, } \\
\text { HAU, EMB }\end{array}$ & WE & WE & WE & & \\
\hline Idaea filicata Нв. * & STAU, RE & WE & WE & $\begin{array}{c}\text { RE, HA, } \\
\text { WE }\end{array}$ & RE, EMB & \\
\hline Idaea moniliata D. \& S. & STAU & & & & & \\
\hline Idaea circuitaria Нв. & & & & $\mathrm{HAU}$ & & \\
\hline Idaea elongaria RAMBUR * & & & & & EMB & \\
\hline
\end{tabular}




\begin{tabular}{|c|c|c|c|c|c|c|}
\hline Artenliste & Korfu & Lefkada & Ithaka & Kefalonia & Zakynthos & $\begin{array}{c}\text { Kythira/ } \\
\text { Kleine Inseln }\end{array}$ \\
\hline Idaea palaestinensis STERNECK & & & & & $\begin{array}{l}\text { EMB, } \\
\text { HAU, } \\
\text { PAPE }\end{array}$ & \\
\hline Idaea politaria Нв. & $\begin{array}{c}\text { STAU, } \\
\text { HAU, KRU }\end{array}$ & & & & & \\
\hline Idaea consolidata LEDERER & & $\mathrm{RE}$ & & & & \\
\hline Idaea seriata SCHRANK & RE, EMB & & & $\mathrm{RE}$ & RE, EMB & \\
\hline Idaea albitorquata PÜNGELER * & & & WE & & & \\
\hline Idaea dimidiata HFN. & & & & HAU & & \\
\hline Idaea camparia H. - S. ${ }^{\star}$ & & WE & & & EMB & \\
\hline Idaea subsericeata HAw. ${ }^{*}$ & $\mathrm{HAU}$ & & & WE & & \\
\hline Idaea infirmaria RAMBUR & $\mathrm{HAU}$ & & & & & \\
\hline Idaea trigeminata Haw. ${ }^{*}$ & RE, EMB & & WE & WE & & \\
\hline Idaea distinctaria BoISDUVAL * & STAU & WE & WE & WE & $\begin{array}{l}\text { HAU, } \\
\text { PAPE }\end{array}$ & \\
\hline Idaea ostrinaria Нв. * & & RE, WE & WE & RE, WE & & \\
\hline Idaea emarginata L. & STAU & & & & & \\
\hline Idaea aversata $\mathrm{L}$. & $\begin{array}{c}\text { DE LA } \\
\text { GARDE }\end{array}$ & & & & & \\
\hline Idaea rubraria STAUDINGER * & & & WE & & & \\
\hline Idaea degeneraria $\mathrm{Hв.}$ & STAU, RE & WE & & $\begin{array}{l}\text { RE, HAU, } \\
\text { WE }\end{array}$ & & \\
\hline Idaea straminata BORKHAUSEN * & STAU & & & WE & & \\
\hline Rhodostrophia calabra PATEGNA * & $\begin{array}{l}\text { STAU, RE, } \\
\text { GINZ }\end{array}$ & WE & WE & $\mathrm{RE}$ & & \\
\hline Rhodometra sacraria L. & $\begin{array}{l}\text { STAU, } \\
\text { DAHL }\end{array}$ & & & & & \\
\hline Casilda antophilaria Нв. * & $\begin{array}{c}\text { STAU, } \\
\text { BUR \& } \\
\text { ILT, HAU }\end{array}$ & GRAF & & & & \\
\hline Cataclysme riguata Нв. & $\begin{array}{c}\text { STAU, } \\
\text { HAU \& } \\
\text { VIID }\end{array}$ & & & & & \\
\hline Orthonama obstipata F. * & $\mathrm{RE}$ & & WE & WE & & \\
\hline Xanthorhoe fluctuata L. * & & & WE & WE & & \\
\hline Catarhoe hortulanaria STAUDINGER * & & & WE & & & \\
\hline Catorhoe permixtaria H. - S. ${ }^{\star}$ & & & WE & WE & & \\
\hline Epirrhoe alternata MÜLLER & $\mathrm{RE}$ & WE & & & & \\
\hline Epirrhoe galiata D. - S. & $\begin{array}{l}\text { HAU \& } \\
\text { VIID }\end{array}$ & & & & & \\
\hline Protorhoe unicata GUENEE * & & RE, WE & WE & WE & & \\
\hline Camptogramma bilineata L. & STAU, RE & & & RE, WE & & \\
\hline Earophila badiata D. \& S. * & $\begin{array}{c}\text { HAU \& } \\
\text { VIID }\end{array}$ & & & & & \\
\hline Coenotephria ablutaria BoIsDUvAL * & $\begin{array}{c}\text { HAU \& } \\
\text { VIID }\end{array}$ & RE, WE & WE & WE & & \\
\hline Cidaria fulvata Forster & $\begin{array}{l}\text { HAU \& } \\
\text { VIID }\end{array}$ & & & & & \\
\hline Thera variata D. \& S. * & $\mathrm{RE}$ & & & WE & & \\
\hline Thera britannica TURNER * & & & & WE & & \\
\hline Thera cupressata GEYER & $\begin{array}{l}\text { RE, HAU } \\
\text { \& VIID }\end{array}$ & & & & & \\
\hline
\end{tabular}




\begin{tabular}{|c|c|c|c|c|c|c|}
\hline Artenliste & Korfu & Lefkada & Ithaka & Kefalonia & Zakynthos & $\begin{array}{c}\text { Kythira/ } \\
\text { Kleine Inseln }\end{array}$ \\
\hline Cidaria fulvata Fonster & $\begin{array}{l}\text { HAU \& } \\
\text { VIID }\end{array}$ & & & & & \\
\hline Horisme vitalbata D. \& S. & $\begin{array}{l}\text { STAU, } \\
\text { HAU \& } \\
\text { VIID }\end{array}$ & & & & & \\
\hline Horisme tersata D. \& S. & $\begin{array}{l}\text { STAU, } \\
\text { HAU \& } \\
\text { VIID }\end{array}$ & & & & & \\
\hline Eupithecia cuculliaria REBEL & & RE, WE & WE & RE, MIR & & \\
\hline Eupithecia irriguata Нв. * & & & & WE & & \\
\hline Eupithecia insigniata Нв. * & & & WE & WE & & \\
\hline Eupithecia centaureata D. \& S. & MIR & & & WE & & \\
\hline Eupithecia schiefereri ВонATsCH * & MIR & & & WE & & \\
\hline Eupithecia quercetica Prout & MIR & & WE & WE & & \\
\hline Eupithecia gratiosata H. - S. & & & & & RE, MIR & \\
\hline Eupithecia breviculata DONZEL & & $\begin{array}{l}\text { RE, MIR, } \\
\text { WE }\end{array}$ & WE & $\begin{array}{l}\text { RE, MIR, } \\
\text { WE }\end{array}$ & & $\begin{array}{c}\text { Meganisi }= \\
\text { RE }\end{array}$ \\
\hline Eupithecia dodoneata GuEnEE * & & WE & WE & WE & & \\
\hline Eupithecia phoeniceata RAMBUR & RE, MIR & & & & & \\
\hline Eupithecia oxycedrata RAMBUR & $\mathrm{RE}$ & & & WE & & \\
\hline Gymnoscelis rufifasciata HAw. & $\begin{array}{l}\text { RE, MIR, } \\
\text { EMB }\end{array}$ & WE & WE & WE & PAPE & \\
\hline Chloroclystis $v$-ata HAw. ${ }^{*}$ & DAHL & & WE & & & \\
\hline Pasiphila rectangulata $\mathrm{L}$. & MIR & & WE & & & \\
\hline Chesias rufata $\mathrm{F}$. & & & & & $\begin{array}{l}\text { HAU \& } \\
\text { VIID }\end{array}$ & \\
\hline Oubolophora internata PÜNGELER * & & & WE & WE & & \\
\hline \multicolumn{7}{|l|}{ Notodontidae } \\
\hline Thaumetopoea processionea L. & MA & & & & & \\
\hline Thaumetopoea solitaria FREYER & & & & & FR \& PI & \\
\hline Thaumetopoea pytiocampa D. \& S. & $\begin{array}{l}\text { STAU, } \\
\text { DAHL }\end{array}$ & WE & & WE & FR \& PI & \\
\hline Cerura bifida BRAHM & STAU & & & & & \\
\hline Drymonia dodonaea D. \& S. & STAU & & & & & \\
\hline Drymonia ruficornis HFN. ${ }^{\star}$ & & & WE & WE & & \\
\hline Drymonia velitaris HFN. & STAU & & & & & \\
\hline Phalera bucephala L. & & FR \& PI & & & & \\
\hline $\begin{array}{l}\text { Phalera bucephaloides } \\
\text { OCHSENHEIMER }\end{array}$ & STAU & FR \& PI & & & & \\
\hline Harpyia milhauseri $\mathrm{F}$. & STAU, RE & FR \& PI & WE & WE & & \\
\hline \multicolumn{7}{|l|}{ Noctuidae } \\
\hline Acronicta psi L. * & & & WE & & & \\
\hline Acronicta aceris $\mathrm{L}$. & $\mathrm{HA}$ & PI & WE & & & \\
\hline Acronicta euphorbiae D. \& S. & & & WE & & PI & \\
\hline Craniophora ligustri D. \& S. & HA & & & & & \\
\hline Simyra albovenosa GoEzE & HA & & & & & \\
\hline Pechipogo plumigeralis Нв. ${ }^{\star}$ & & & WE & & MA & \\
\hline Catocala conversa EsPER & HA & & & & & \\
\hline Catocala nymphagoga EsPER & & & WE & MA & & \\
\hline Ephesia disjuncta GEYER & HA & & & & & \\
\hline Ephesia eutychea Treitschкe * & $\begin{array}{l}\text { STAU, HA, } \\
\text { EMB, KRU }\end{array}$ & MA, PI & WE & MA, WE & & HA \\
\hline
\end{tabular}




\begin{tabular}{|c|c|c|c|c|c|c|}
\hline Artenliste & Korfu & Lefkada & Ithaka & Kefalonia & Zakynthos & $\begin{array}{c}\text { Kythira/ } \\
\text { Kleine Inseln }\end{array}$ \\
\hline Minucia lunaris D. \& S. & HA & & WE & WE & & \\
\hline Ophiusa tirhaca CRAMER & $\mathrm{HA}$ & PI & WE & WE & & \\
\hline Dysgonia algira $\mathrm{L}$. & HA & PI & WE & $\begin{array}{l}\mathrm{HA}, \mathrm{MA}, \\
\text { WE }\end{array}$ & & $\mathrm{HA}$ \\
\hline Grammodes bifasciata PetagnA * & & PI & WE & & & \\
\hline Prodotis stolida $\mathrm{F}$ & HA & & & & & \\
\hline Lygephila craccae D. \& S. & & PI & & & & \\
\hline Apopestes spectrum EsPER & & PI & & & & \\
\hline Autophila dilucida Нв. & HA & & WE & & & \\
\hline Catephia alchymista D. \& S. * & & PI & WE & WE & & \\
\hline Aedia leucomelas L. & & PI & & & PAPE & \\
\hline Tyta luctuosa D. \& S. & $\begin{array}{c}\text { DE LA } \\
\text { GARDE, } \\
\text { HA, } \\
\text { DAHL }\end{array}$ & HA, PI & & WE & & \\
\hline Zethes insularis RAMBUR & RE, GINZ & $\begin{array}{l}\text { HA, WE, } \\
\text { PI }\end{array}$ & WE & WE & & \\
\hline Zekelita antiqualis Нв. & HA, GINZ & & WE & HA, WE & & \\
\hline Hypena obsitalis Нв. & HA & & & & & \\
\hline Hypena lividalis Нв. & HA, KLEW & & & & & \\
\hline Araeopteron ecphaea Hampson * & DAHL & & & & & \\
\hline Zebeeba falsalis H. - S. * & & & WE & & & \\
\hline Eutelia adulatrix HB. & & PI & WE & HA, WE & & \\
\hline Autographa gamma L. & $\begin{array}{c}\text { HA, } \\
\text { DAHL }\end{array}$ & HA, PI & WE & WE & & \\
\hline Trichoplusia ni Нв. & HA & PI & WE & & PAPE & \\
\hline Trichoplusia circumscripta FreYer * & HA & & WE & WE & & \\
\hline Chrysodeixis chalcites EsPER & HA & & WE & & & \\
\hline Abrostola trigemina WERNEBURG & & & WE & & & \\
\hline Emmelia trabealis Scopoli & $\begin{array}{c}\text { HA, } \\
\text { DAHL }\end{array}$ & & & & & \\
\hline Acontia lucida HFN. & HA & & WE & HA & & \\
\hline Ozarba moldavicola H. - S. & HA & & & & $\mathrm{HA}$ & \\
\hline Ozarba lascivalis LEDERER & HA & & & & & \\
\hline Eublemma ostrina Нв. & HA & & & & & $\mathrm{HA}$ \\
\hline Eublemma parva Нв. & HA & & & & & \\
\hline Eublemma amoепа Нв. & $\mathrm{HA}$ & & & & & \\
\hline Eublemma ragusana FREYER & & $\mathrm{HA}$ & & & & \\
\hline Glossodice polygramma DUPONCHEL * & & & WE & WE & & \\
\hline Metachrostis velox Нв. * & DAHL & & & & & \\
\hline Metachrostis dardouini BoIsDuvaL & & $\mathrm{HA}$ & & & & \\
\hline Xanthodes albago F. & & PI & & & & \\
\hline Cucullia umbratica L. & HA & & & & & \\
\hline Cucullia blattariae EsPER * & & & WE & & & \\
\hline Calocucullia celsiae H. - S. & $\mathrm{HA}$ & & & & & \\
\hline Calophasia platyptera EsPER * & & PI & & WE & & \\
\hline Omphalophana anatolica LEDERER * & & WE & WE & WE & & \\
\hline Amephana dalmatica REBEL * & & & WE & WE & & \\
\hline Pyrois effusa BoIsduval & HA & PI & WE & & & MA \\
\hline Aegle semicana EsPER & HA & & WE & & & \\
\hline Protoschinia scutosa D. \& S. & $\mathrm{HA}$ & & & & & \\
\hline Heliothis viriplaca HFN. & HA & & & & & \\
\hline
\end{tabular}




\begin{tabular}{|c|c|c|c|c|c|c|}
\hline Artenliste & Korfu & Lefkada & Ithaka & Kefalonia & Zakynthos & $\begin{array}{c}\text { Kythira/ } \\
\text { Kleine Inseln }\end{array}$ \\
\hline Heliothis peltigera D. \& S. & HA & & & & & \\
\hline Heliotis nubigera H. - S. & & & & & & HA \\
\hline Helicoverpa armigera Нв. & & PI & WE & WE & & \\
\hline Brithis crini $\mathrm{F}$ & HA & & & & & \\
\hline Elaphria venustula Нв. & HA & & & & & \\
\hline Caradrina clavipalpis ScoP. & RE, HA & & WE & WE & & \\
\hline Caradrina wullschlegeli PÜNGELER ${ }^{*}$ & & & WE & & & \\
\hline Caradrina flavirena GUENEE ${ }^{*}$ & & PI & WE & WE & & \\
\hline Hoplodrina superstes OCHSENHEIMER * & & WE & & & & \\
\hline Hoplodrina ambigua D. \& S. ${ }^{*}$ & & WE & WE & WE & & \\
\hline Charanyca trigrammica HFN. ${ }^{*}$ & & WE & & & & \\
\hline Spodoptera exiguа Нв. & HA & WE & WE & & $\begin{array}{l}\text { EMB, } \\
\text { PAPE }\end{array}$ & \\
\hline Spodoptera cilium GuENEE & DAHL & PI & & & & \\
\hline Sesamia nonagrioides LEFEBVRE & DAHL & PI & & MA, WE & & \\
\hline Athetis hospes Freyer & HA & & WE & & & \\
\hline Mormo maura L. & HA, MA & & & & & \\
\hline Polyphaenis sericata EsPER & HA & & & & & \\
\hline Thalpophila matura HFN. & $\begin{array}{l}\text { HA, MA, } \\
\text { DAHL }\end{array}$ & PI & & & & \\
\hline Phlogorophora meticulosa $\mathrm{L}$. & HA & & WE & & HA & \\
\hline Chloantha hyperici D. \& S. * & & PI & WE & WE & & \\
\hline Callopistria juventina STOLL & & & & & HA & \\
\hline Callopistria latreillei DUPONCHEL & $\mathrm{HA}, \mathrm{MA}$ & WE & WE & WE & & \\
\hline Agrochola lychnites D. \& S. & HA, MA & & & & & \\
\hline Spudaea pontica KLjuтsснко & HA & & & & & \\
\hline Conistra vaccinii $\mathrm{L} .{ }^{*}$ & & & & WE & & \\
\hline Aporophila australis BOISDUVAL & HA, MA & & & & & \\
\hline Aporophila nigra HAw. & HA & & & & & \\
\hline Lithophane ledereri STAUDINGER * & & & & WE & & \\
\hline Xylena vetusta Нв. & HA & & & & & \\
\hline Dryobotodes carbonis WAGNER & MA & & & & & \\
\hline Dryobotodes tenebrosa EsPER & & & & MA & & MA \\
\hline Trigonophora flammea EsPER & $\mathrm{HA}, \mathrm{MA}$ & & & & & \\
\hline Polymixis serpentina TReITsChKe & STAU, HA & & & HA, MA & & \\
\hline Polymixis rufocincta GEYER & HA & & & & & \\
\hline Polymixis culoti SCHAWERDA & HA & & & & & \\
\hline Blepharita solieri BOISDUVAL & HA & PI & & & & HA \\
\hline Abromia sicula Turati * & & WE & WE & WE & & \\
\hline Oligia latruncula D. \& S. * & & & WE & & & \\
\hline Mesapamea secalis L. & & PI & & & & \\
\hline Luperina dumerilii DUPONCHEL & HA, MA & & & & & \\
\hline Discestra trifolii HFN. & & PI & & & & \\
\hline Hada plebeja L. * & STAU & & & & & \\
\hline Hecatera bicolorata HFN. ${ }^{*}$ & & WE, PI & WE & & & \\
\hline Conisania luteago D. \& S. ${ }^{\star}$ & & & WE & WE & & \\
\hline Hadena adriana SCHAWERDA * & & & WE & & & \\
\hline Hadena magnolii BoIsDUvAL ${ }^{*}$ & & & WE & & & \\
\hline Hadena perplexa D. \& S. ${ }^{*}$ & & & & WE & & \\
\hline Hadena syriaca OSTHELDER * & & WE & & & & \\
\hline Mythimna vitellina Нв. & HA & PI & WE & & & HA \\
\hline
\end{tabular}




\begin{tabular}{|c|c|c|c|c|c|c|}
\hline Artenliste & Korfu & Lefkada & Ithaka & Kefalonia & Zakynthos & $\begin{array}{c}\text { Kythira/ } \\
\text { Kleine Inseln }\end{array}$ \\
\hline Leucania putrescens Нв. & HA & PI & & & & \\
\hline Mythimna congrua Нв. & $\begin{array}{c}\text { HA, } \\
\text { DAHL }\end{array}$ & & & & & \\
\hline Mythimna l-album L. & HA & & & WE & & \\
\hline Mythimna sicula TreiTsChKe & HA & WE, PI & WE & WE & & \\
\hline Mythimna alopecuri BoIsDUvAL * & & & WE & & & \\
\hline Mythimna prominens WALKER & HA & & WE & & & \\
\hline Mythimna riparia RAMBUR * & DAHL & & & & & \\
\hline Mythimna loreyi DUPONCHEL & HA & PI & & & & \\
\hline Mythimna unipuncta HAw. ${ }^{*}$ & & PI & WE & & & \\
\hline Orthosia incerta HFN. * & & & & WE & & \\
\hline Orthosia cruda D. \& S. & HA & WE & WE & WE & & \\
\hline Orthosia miniosa D. \& S. * & & WE & & WE & & \\
\hline Orthosia cerasi $\mathrm{F}^{*}$ & & & WE & WE & & \\
\hline \multicolumn{7}{|l|}{ Orthosia dalmatica WAGNER * } \\
\hline Orthosia rorida FRIVALDSZKY & HA & & & WE & & \\
\hline Egira conspicillaris L. & HA & & WE & WE & & \\
\hline Ochropleura leucogaster FREYER & & PI & & HA & & \\
\hline Noctua pronuba L. * & & WE, PI & WE & WE & & \\
\hline Noctua interposita Нв. & & PI & & & & \\
\hline Noctua comes $\mathrm{L}$. & HA & PI & WE & $\begin{array}{l}\text { HA, MA, } \\
\text { WE }\end{array}$ & & \\
\hline Noctua fimbriata SCHREBER & & PI & & & & \\
\hline Noctua janthina D. \& S. & HA & & & & & \\
\hline Xestia xanthographa D. \& S. & MA & & & MA & & MA \\
\hline Rhyacia saucia Нв. & HA & PI & WE & & & \\
\hline Agrotis bigramma ESPER & & PI & & & & \\
\hline Agrotis puta Нв. & HA & & WE & $\begin{array}{l}\text { HA, MA, } \\
\text { WE }\end{array}$ & & \\
\hline Agrotis ipsilon $\mathrm{HFN} .{ }^{*}$ & & PI & WE & WE & & \\
\hline Agrotis trux Нв. & & PI & & & & \\
\hline Agrotis clavis HFN. & HA & & & & & \\
\hline Agrotis segetum D. \& S. & HA, MA & PI & & WE & & \\
\hline Agrotis spinifera Нв. ${ }^{*}$ & DAHL & & & & & \\
\hline \multicolumn{7}{|l|}{ Lymantridae } \\
\hline Lymantria dispar $\mathrm{L}$. & GINZ & $\begin{array}{l}\text { RE, WE, } \\
\text { FR \& PI }\end{array}$ & WE & WE & FR \& PI & \\
\hline Ocneria rubea D. \& S. * & DAHL & & & & & \\
\hline Ocneria ledereri MILliere & & FR \& PI & & & & \\
\hline Parocneria terebynthi FREYER & STAU & $\begin{array}{c}\text { RE, FR \& } \\
\text { PI }\end{array}$ & & & & \\
\hline Euproctis chrysorrhoea L. & STAU & & & & & \\
\hline Arctornis l-nigrum MÜLLER & & FR \& PI & & & & \\
\hline \multicolumn{7}{|l|}{ Nolidae } \\
\hline Meganola togatulalis Нв. ${ }^{*}$ & & FR \& PI & WE & & & \\
\hline Meganola strigula D. \& S. & & FR \& PI & & & & \\
\hline Nola cicatricalis TREITSCHKE ${ }^{*}$ & & & & WE & & \\
\hline Nola subchlamidula STAUDINGER * & STAU, RE & FR \& PI & & WE & & \\
\hline Nycteola revayana SCOPOLI * & & & & WE & & \\
\hline Nycteola columbana TURNER * & & & WE & WE & & \\
\hline Nycteola asiatica KRULIKOVSKY & EMB & PI & & & & \\
\hline
\end{tabular}




\begin{tabular}{|c|c|c|c|c|c|c|}
\hline Artenliste & Korfu & Lefkada & Ithaka & Kefalonia & Zakynthos & $\begin{array}{l}\text { Kythira/ } \\
\text { Kleine Inseln }\end{array}$ \\
\hline Nycteola siculana FucHs & & PI & & & & \\
\hline Bena prasinana L. & & PI & & & & \\
\hline \multicolumn{7}{|l|}{ Arctiidae } \\
\hline Cybosia mesomella L. & STAU & & & & & \\
\hline Eilema depressa EsPER * & STAU & & & WE & & \\
\hline Eilema complana L. & STAU & & & & & \\
\hline Eilema costalis ZELLER * & & WE & WE & WE & & \\
\hline Eilema caniola Нв. & RE, DAHL & $\begin{array}{l}\text { FR \& PI, } \\
\text { WE }\end{array}$ & WE & MA, WE & & MA \\
\hline Setina irrorella L. & STAU & & & & & \\
\hline Amata phegea L. & STAU & & & & & \\
\hline Amata marjana STAUDER * & & & & WE & & \\
\hline Dysauxes famula Freyer * & $\begin{array}{l}\text { STAU, KÖ, } \\
\text { HU }\end{array}$ & & WE & WE & & \\
\hline Spiris striata L. & STAU & & & & FR \& PI & \\
\hline Utetheisa pulchella L. ${ }^{*}$ & DAHL & & & & & \\
\hline Ocnogyna parasita Нв. & & & & & LU, WI & \\
\hline Phragmatobia fuliginosa L. & $\begin{array}{l}\text { STAU, MA, } \\
\text { DAHL }\end{array}$ & MA, PI & & MA & & \\
\hline Cymbalophora pudica EsPER & $\begin{array}{l}\text { STAU, } \\
\text { DAHL } \\
\end{array}$ & $\begin{array}{l}\text { RE, FR \& } \\
\text { PI }\end{array}$ & & & & \\
\hline Spilosoma lubricipeda L. ${ }^{*}$ & DAHL & & & & & \\
\hline Diacrisia sannio L. & STAU & & & & & \\
\hline Arctia villica $\mathrm{L}$. & $\begin{array}{c}\text { STAU, } \\
\text { DE LA } \\
\text { GARDE }\end{array}$ & $\begin{array}{l}\text { WE, FR } \\
\quad \& \mathrm{PI}\end{array}$ & WE & WE & & \\
\hline Arctia festiva HFN. * & & & & STAU, WE & & \\
\hline Callimorpha quadripunctaria PoDA & STAU, RE & & & & & $\mathrm{KA} \& \mathrm{KO}$ \\
\hline Tyria jacobaeae L. & $\begin{array}{c}\text { STAU, } \\
\text { DE LA } \\
\text { GARDE }\end{array}$ & & & & & \\
\hline \multicolumn{7}{|l|}{ Trichoptera } \\
\hline \multicolumn{7}{|l|}{ Ptilopotamidae } \\
\hline Wormaldia spec. & & & & MA, WE & & \\
\hline \multicolumn{7}{|l|}{ Polycentropodidae } \\
\hline Polycentropus ierapetra MALICKY ${ }^{*}$ & MA & & & MA,WE & MA & MA \\
\hline \multicolumn{7}{|l|}{ Psychomyiidae } \\
\hline Tinodes braueri MCLACHLAN * & MA & & & MA, WE & & \\
\hline \multicolumn{7}{|l|}{ Hydropsychidae } \\
\hline Hydropsyche instabilis CurTis * & & & & MA, WE & & \\
\hline $\begin{array}{l}\text { Hydropsyche peristerica Botos. \& } \\
\text { MARINK. * }\end{array}$ & & MA & & MA, WE & MA & \\
\hline \multicolumn{7}{|l|}{ Limnephilidae } \\
\hline Micropterna testacea GMELIN * & & & & MA, WE & & \\
\hline Stenophylax mitis MCLACHLAN * & & MA, WE & & MA, WE & & \\
\hline \multicolumn{7}{|l|}{ Leptoceridae } \\
\hline Adicella syriaca ULMER & MA & MA & & MA, WE & & \\
\hline Triaenodes ochreellus McLACHLAN * & MA & MA & & MA, WE & & \\
\hline
\end{tabular}


Tab. 2: Neighbor-joining-tree der genetischen Differenzen von Bankesia Tutт, 1899.

\begin{tabular}{|c|c|c|c|c|c|c|c|}
\hline & 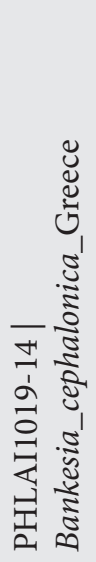 & 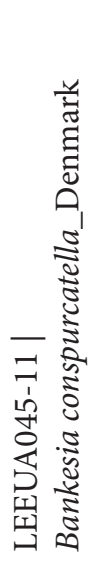 & 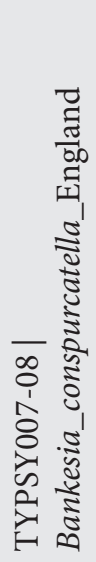 & 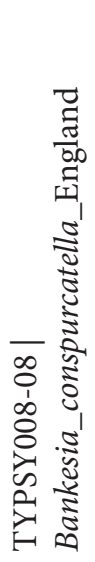 & 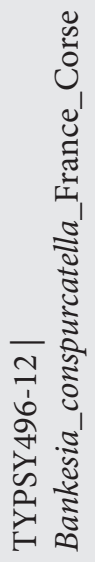 & 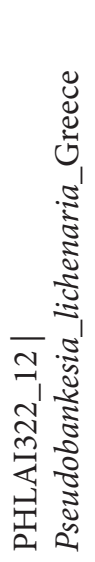 & 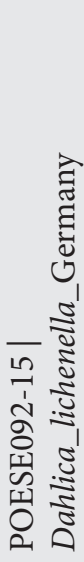 \\
\hline \multicolumn{8}{|l|}{ PHLAI1019-14 | Bankesia_cephalonica_Greece } \\
\hline LEEUA045-11 | Bankesia_conspurcatella_Denmark & 0,07 & & & & & & \\
\hline TIPSY007-08 | Bankesia_conspurcatella_England & 0,06 & 0,00 & & & & & \\
\hline TIPSY008-08 | Bankesia_conspurcatella_England & 0,06 & 0,00 & 0,00 & & & & \\
\hline TIPSY496-12 | Bankesia_montanella_France_Corse & 0,27 & 0,29 & 0,29 & 0,29 & & & \\
\hline PHLAH322-12 | Pseudobankesia_lichenaria_Greece & 0,36 & 0,38 & 0,38 & 0,38 & 0,38 & & \\
\hline POESE092-15 | Dahlica_lichenella_Germany & 0,75 & 0,78 & 0,79 & 0,79 & 0,79 & 0,73 & \\
\hline
\end{tabular}

